\title{
Mycobacterium tuberculosis PanD structure-function analysis and identification of a potent pyrazinoic acid-derived enzyme inhibitor
}

Priya Ragunathan ${ }^{1, \#}$, Malcolm Cole ${ }^{2, \#}$, Chitra Latka ${ }^{1, \#}$, Wassihun Wedajo Aragaw ${ }^{3, \#}$, Pooja Hegde ${ }^{2}$, Joon Shin ${ }^{1}$, Malathy Sony Subramanian Manimekalai ${ }^{1}$, Sankaranarayanan Rishikesan ${ }^{1}$, Courtney C. Aldrich $^{2, *}$, Thomas Dick ${ }^{3,4,5, *}$ and Gerhard Grüber ${ }^{1, *}$

1School of Biological Sciences, Nanyang Technological University, 60 Nanyang Drive, Singapore 637551, Republic of Singapore

2University of Minnesota, College of Pharmacy, Department of Medicinal Chemistry 8-101 Weaver-Densford Hall 308 Harvard St. SE, Minneapolis, MN 55455, USA

3Center for Discovery and Innovation, Hackensack Meridian Health, 111 Ideation Way, Nutley, New Jersey 07110, USA

4Department of Medical Sciences, Hackensack Meridian School of Medicine, 123 Metro Boulevard, Nutley, New Jersey 07110, USA

5Department of Microbiology and Immunology, Georgetown University, 3900 Reservoir Road NW MedicalDental Building, Washington, DC 20007, USA

\#Authors contributed equally to this work

*To whom correspondence should be addressed: Prof. Dr. Courtney Aldrich, Email: aldri015@umn.edu; Prof. Dr. Thomas Dick; Thomas.Dick@hmh-cdi.org; Prof. Dr. Gerhard Grüber, E-mail: ggrueber@ntu.edu.sg

KEYWORDS: Tuberculosis, Mycobacteria, pyrazinamide, pyrazinoic acid, coenzyme A, aspartate decarboxylase 


\section{Supporting Information}

\section{Table of Contents}

\section{Part I: Biological data}

Supplementary Figure S1

Supplementary Figure S2

Supplementary Figure S3

Supplementary Figure S4

Supplementary Figure S5

Supplementary Figure S6

Supplementary Figure S7

Supplementary Figure S8

Supplementary Table 1

Supplementary Table 2

Supplementary Table 3A

Supplementary Table 3B

\section{Part II: Synthetic Chemistry.}

$\begin{array}{ll}\text { General Remarks } & \text { S18 }\end{array}$

POA Analogue Syntheses and Analytical Data $\quad$ S18

$\begin{array}{ll}\text { Esterification of 3-chloro and 3-aminopyrazine } & \text { S18 }\end{array}$

$\begin{array}{ll}\text { General amidation procedure and products } & \text { S19 }\end{array}$

$\begin{array}{ll}\text { General amination procedure and products } & \text { S20 }\end{array}$

$\begin{array}{ll}\text { General Suzuki-Miyaura coupling procedure and products } & \text { S22 }\end{array}$

$\begin{array}{ll}\text { General saponification procedure and final products } & \text { S23 }\end{array}$

$\begin{array}{lr}\text { Part III: References } & \text { S26 }\end{array}$

$\begin{array}{lr}\text { Part IV: NMR Spectra } & \text { S27 }\end{array}$ 


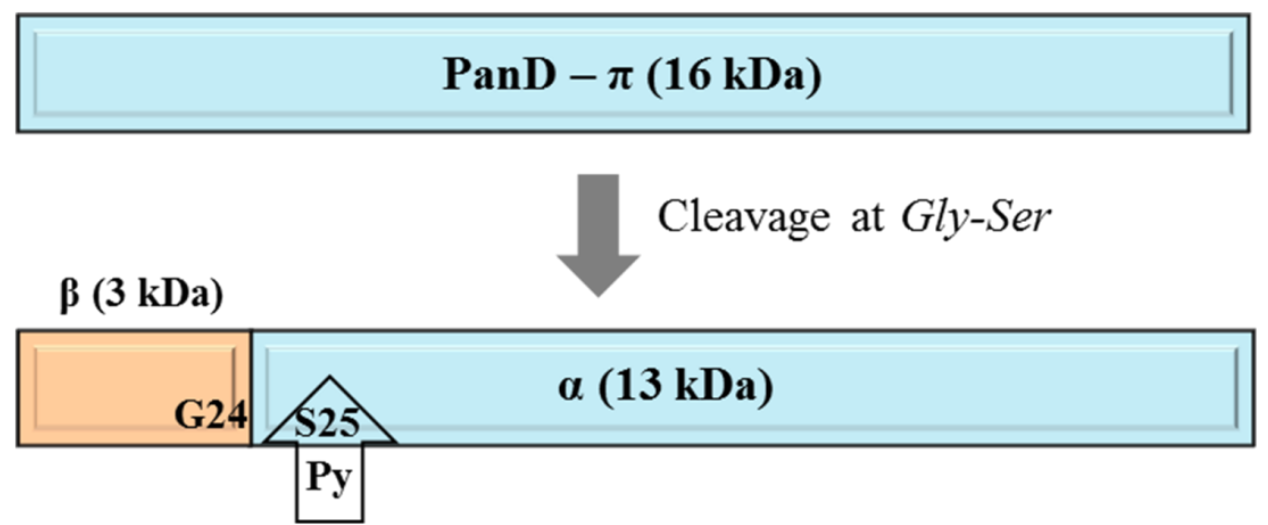

Supplementary Figure S1: Schematic diagram showing the cleavage of PanD $-\pi$ (16 kDa) at G24-S25 leaving behind pyruvoyl group at S25 ( $\alpha$-subunit) and hydroxyl group at G24 ( $\beta$ subunit). 
(A)

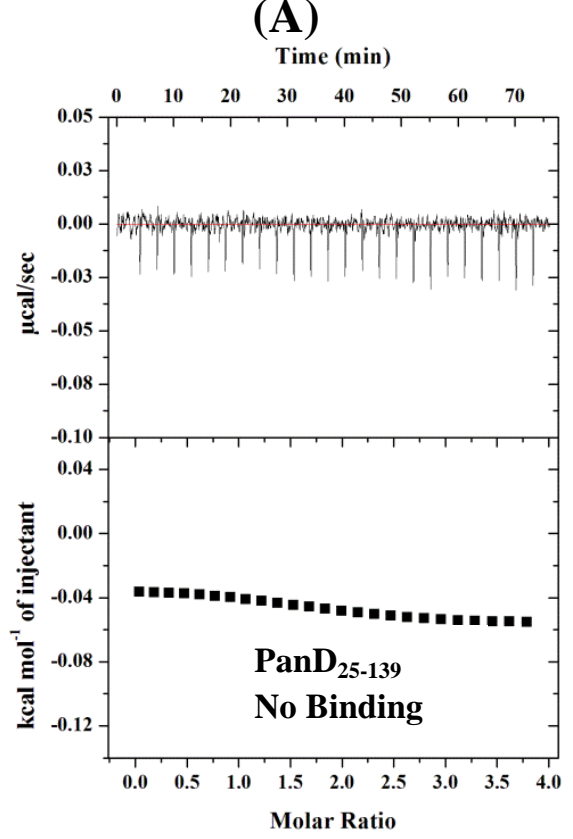

(C)

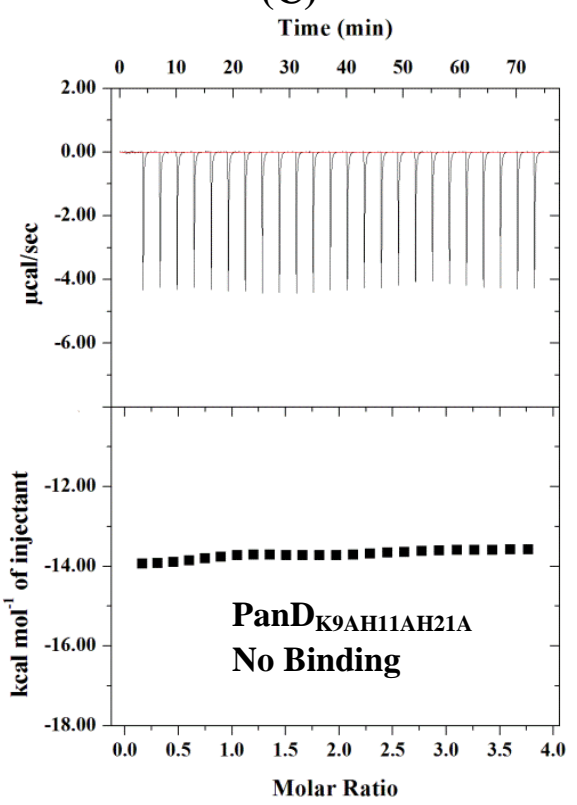

(B)

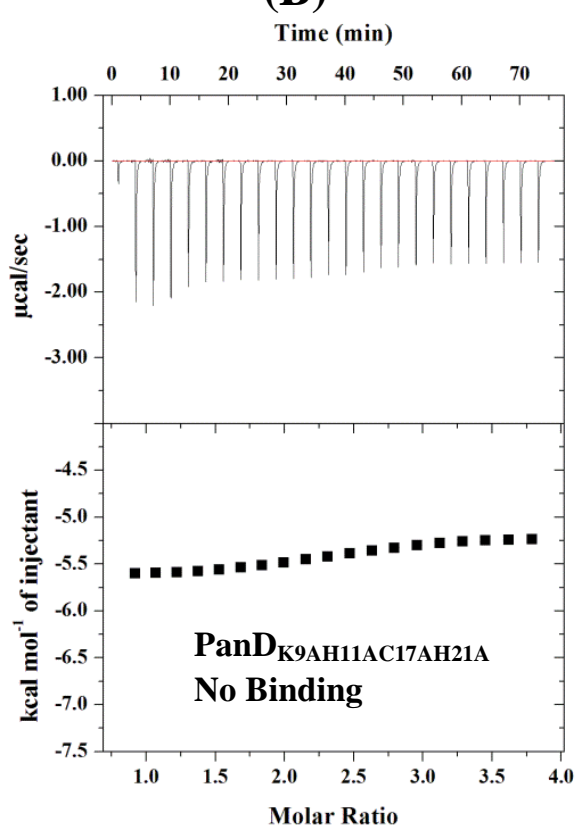

(D)

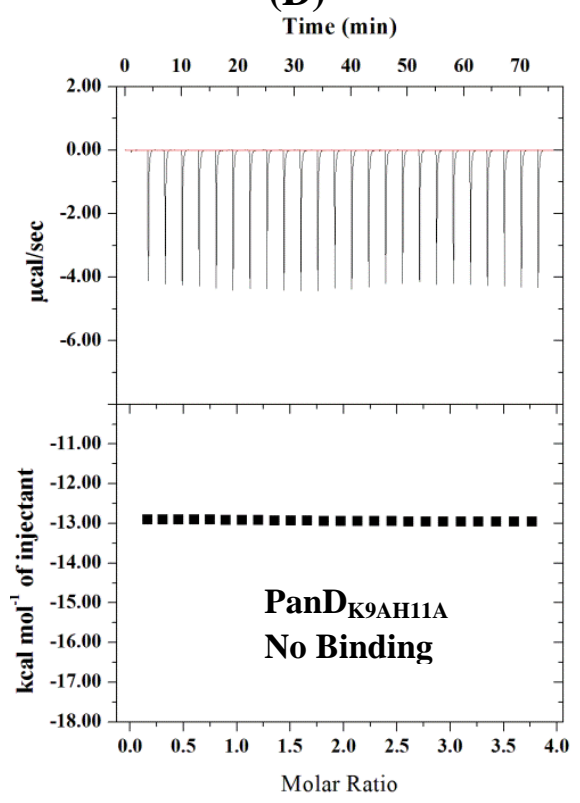

Supplementary Figure S2: Effect of N-terminal mutations in $M t b$ PanD on the binding affinity of POA. Representative ITC profiles are shown for POA with PanD $_{25-239}$, PanD K9AH11AC17AH21A, PanD $_{\text {K9AH11AH21A, and PanD }}$ K9AH11A, respectively. All the four mutants lost their ability to bind to POA. 
(A)

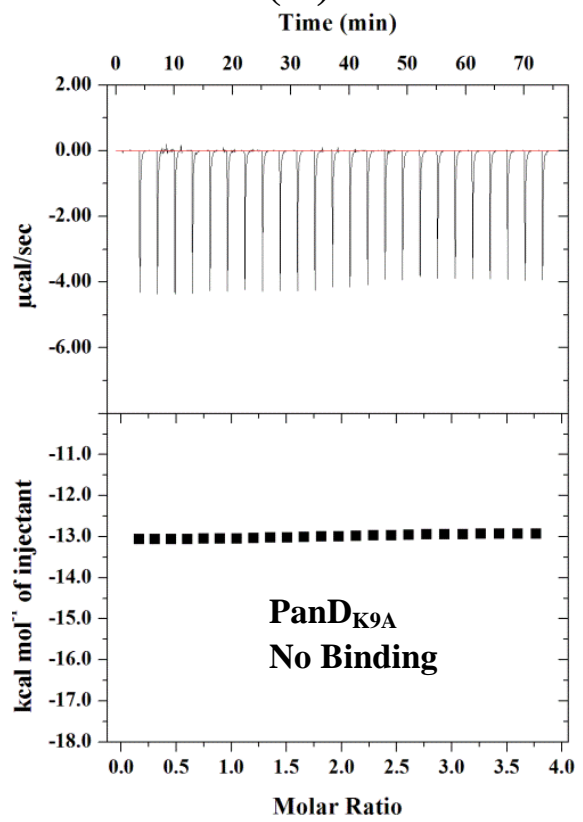

(C)

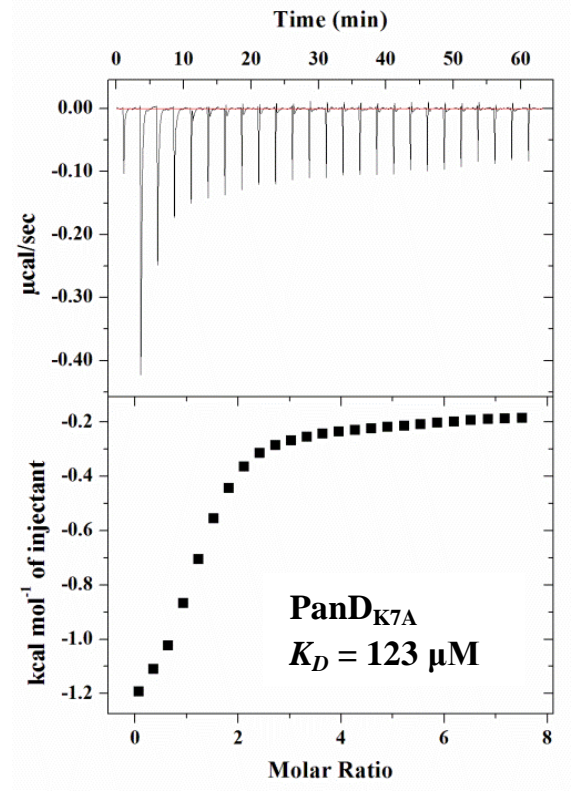

(B)

Time (min)

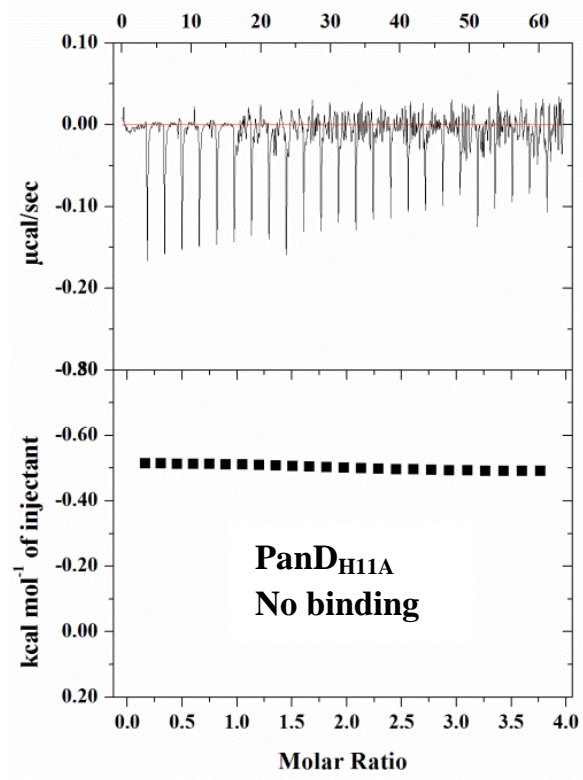

(D)

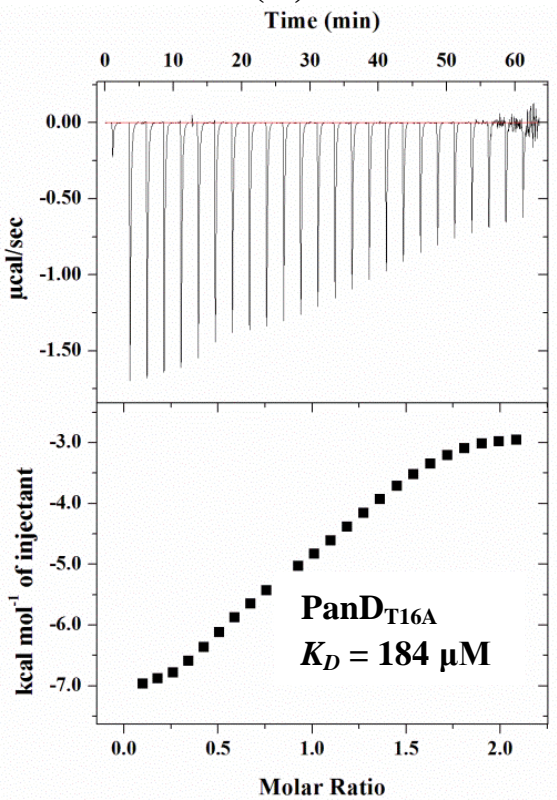


(E)

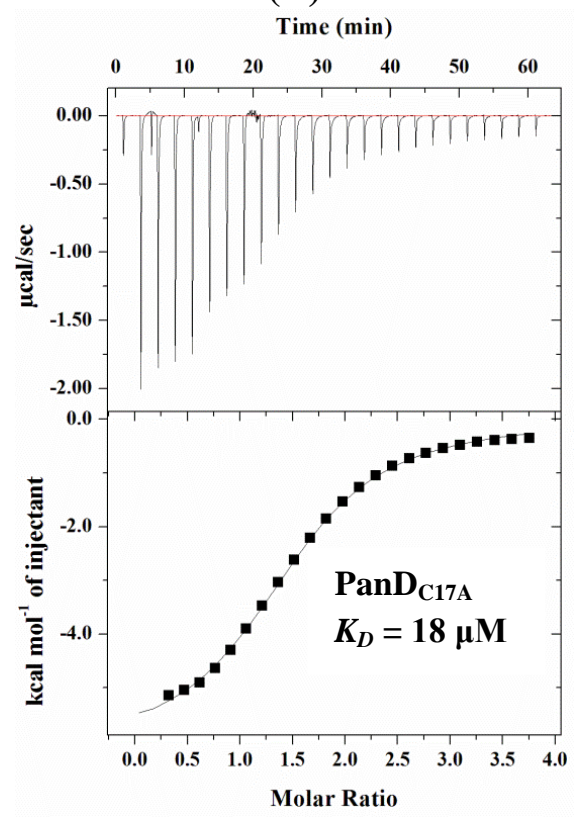

(F)

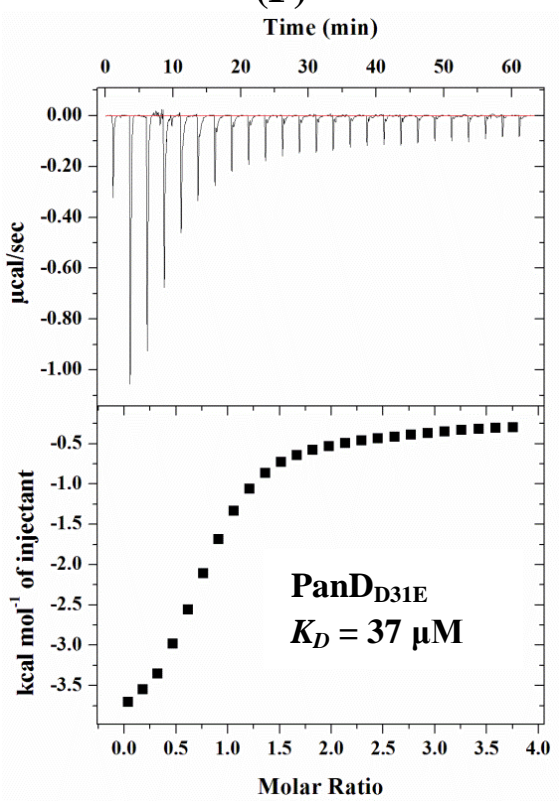

Supplementary Figure S3: Effects of N-terminal single mutations in Mtb PanD on POAbinding. Representative ITC profiles are presented for POA with $\mathrm{PanD}_{\mathrm{K} 9 \mathrm{~A}}$, and $\mathrm{PanD}_{\mathrm{H} 11 \mathrm{~A}}$. Substituting L9 and H11 resulted in a total loss of POA-binding. In comparison, the mutants PanD $\mathrm{K}_{\mathrm{KA}}$ and PanD $\mathrm{T}_{16 \mathrm{~A}}$ showed weaker binding, while the mutants $\mathrm{PanD}_{\mathrm{C} 17 \mathrm{~A}}$ and $\mathrm{PanD}_{\mathrm{D} 31 \mathrm{E}}$ revealed similar binding traits compared to the WT enzyme. 
(A)

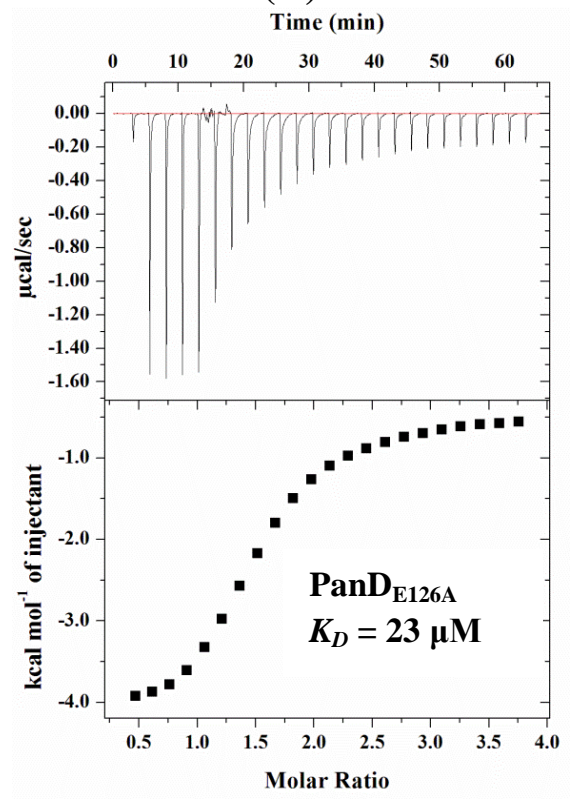

(B)

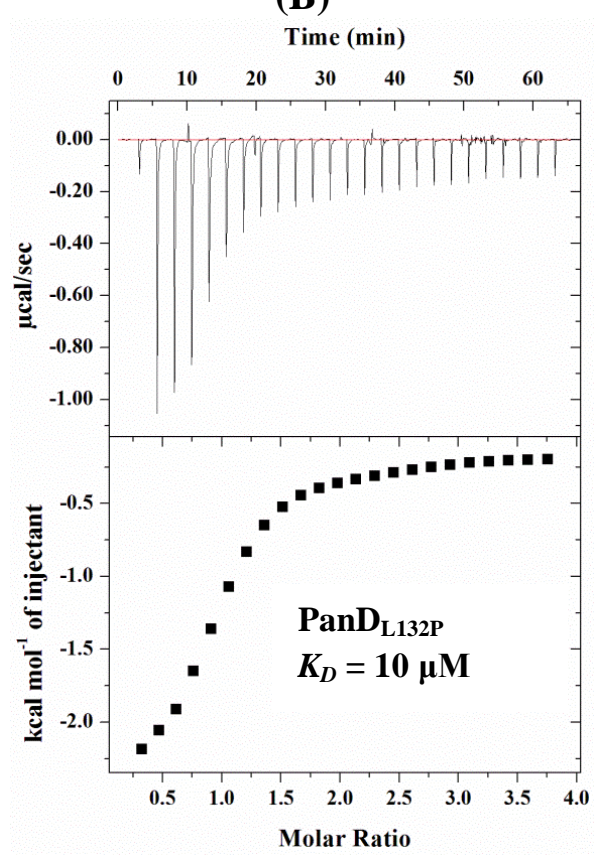

Supplementary Figure S4: POA-binding to the C-terminal mutants $\mathrm{PanD}_{\mathrm{E} 126 \mathrm{~A}}$, and

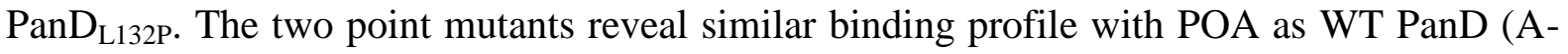
B). The top panel in each figure shows the injection profile after baseline correction. The corresponding bottom panel shows the integration (heat release) for each injection. The solid lines in the bottom panel reveal the fit of the data to a function based on a one-site binding model. 
(A)

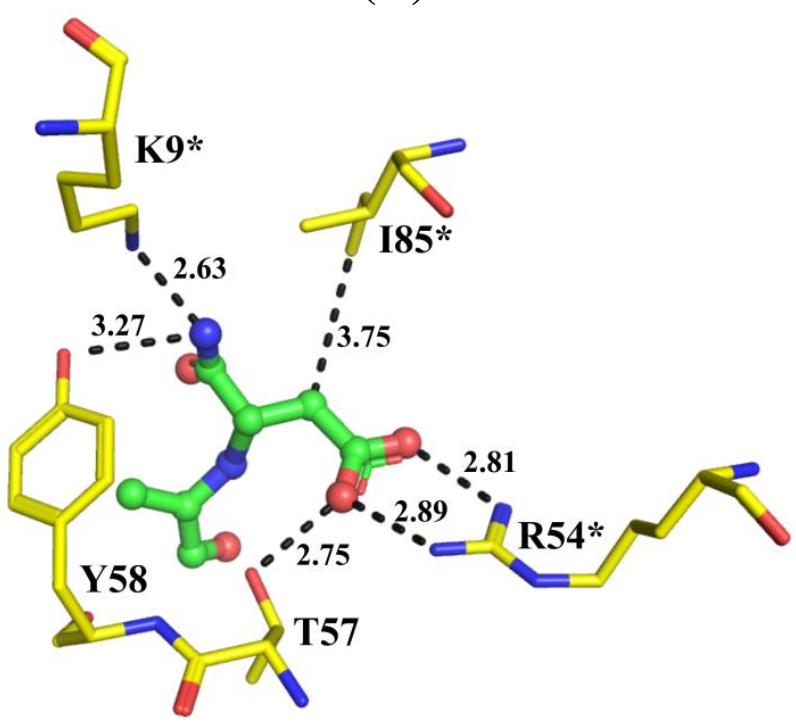

(B)

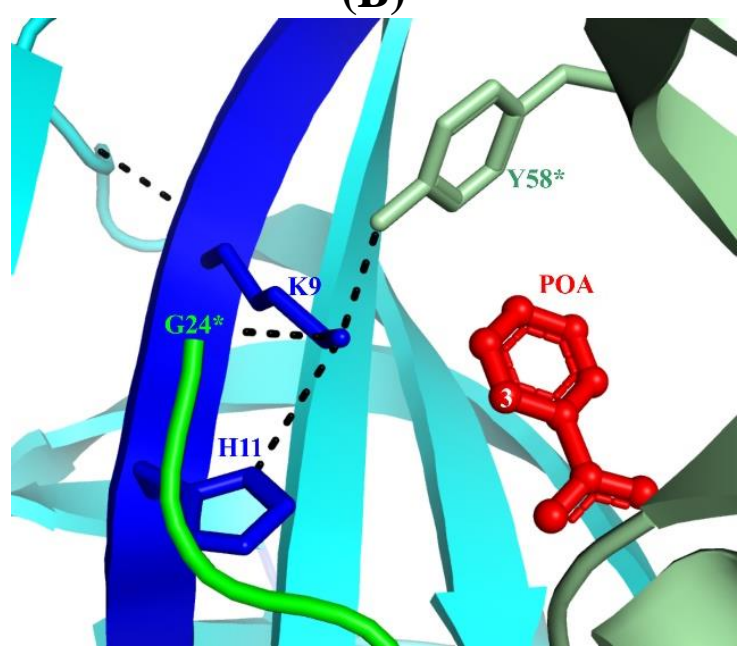

Supplementary Figure S5: $(A)$ Interaction of isoasparagine (green) in the structure of $H$. pylori PanD complex (PDB ID: 1UHE; (1)). Residues which come from the adjacent subunits are marked by an asterisk. (B) POA has hydrophobic interaction with K9 (4.2 A) within the POA-bound Mtb PanD structure (6OYY; (2)). Interestingly, no interaction of the drug occurs with the enzymatically critical $\mathrm{H} 11$ residue, leaving sufficient space at position three of POA to extend, and to generate interaction with H11. Such interaction may also affect the network of K9, H11 and amino acids Y58, G24 of the adjacent subunit, which belong to the catalytic center and contribute to the dimerization/oligomerization. 
(A)

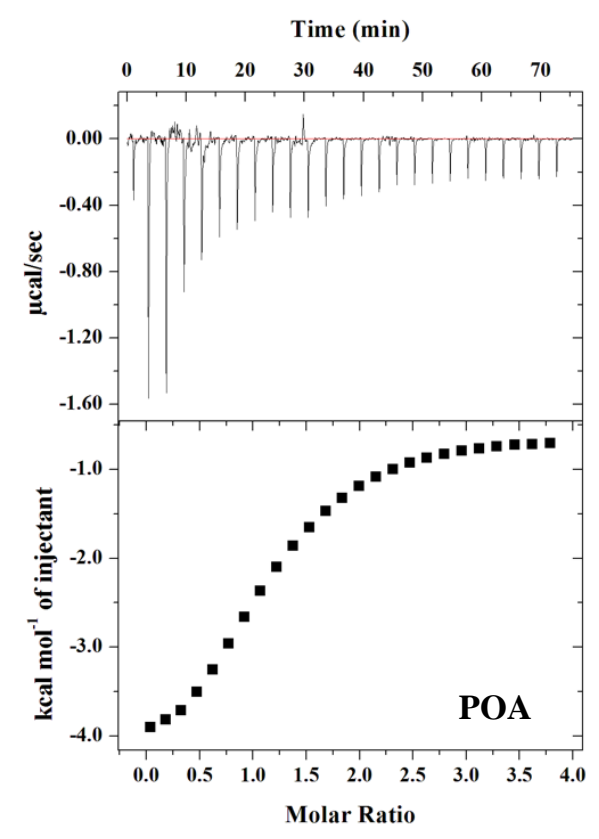

(C)

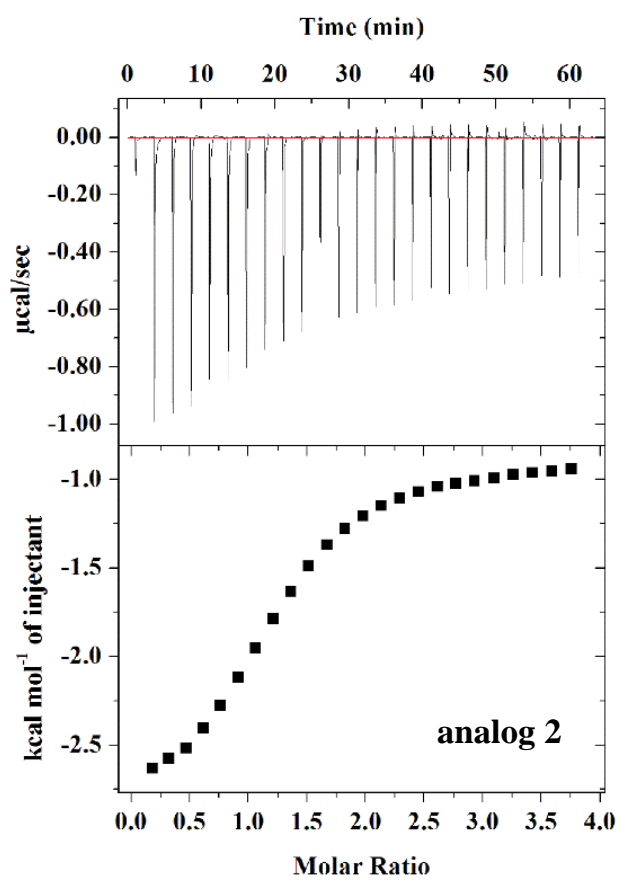

(B)

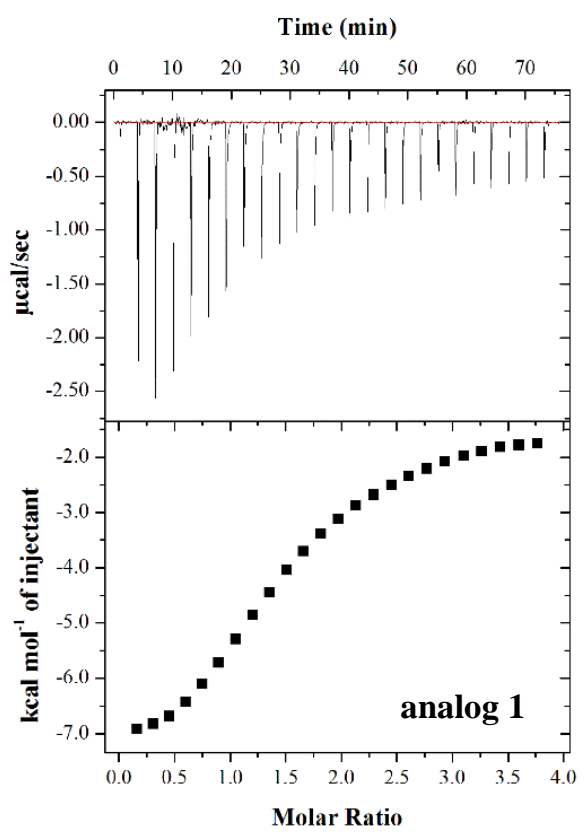

(D)

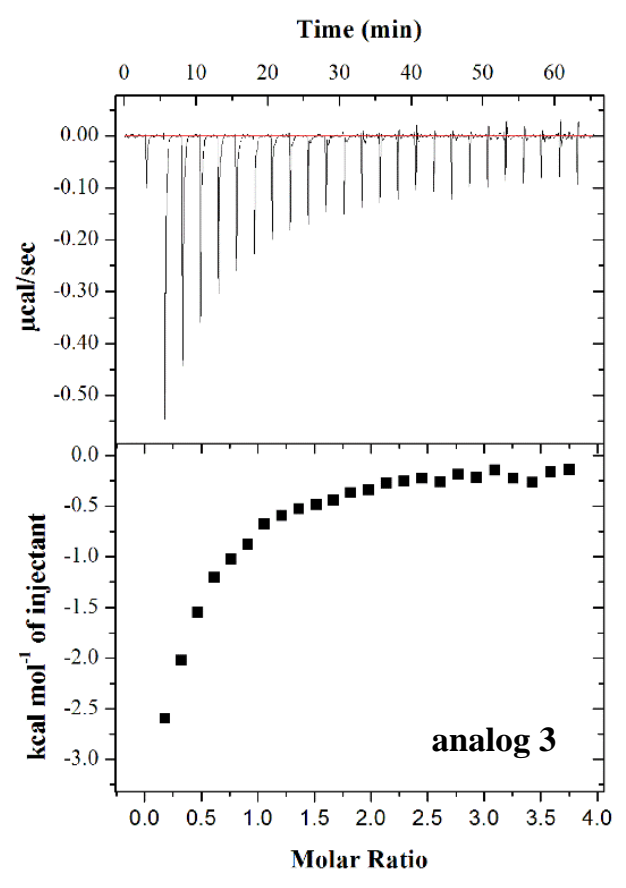

Supplementary Figure S6: Binding profiles of $M t b$ PanD with POA and its analogs. ITC profiles for WT $M t b$ PanD with $2 \mathrm{mM}$ of POA $(A)$, analog $\mathbf{1}(B),-\mathbf{2}(C)$ or $\mathbf{- 3}(D)$. 
(E)

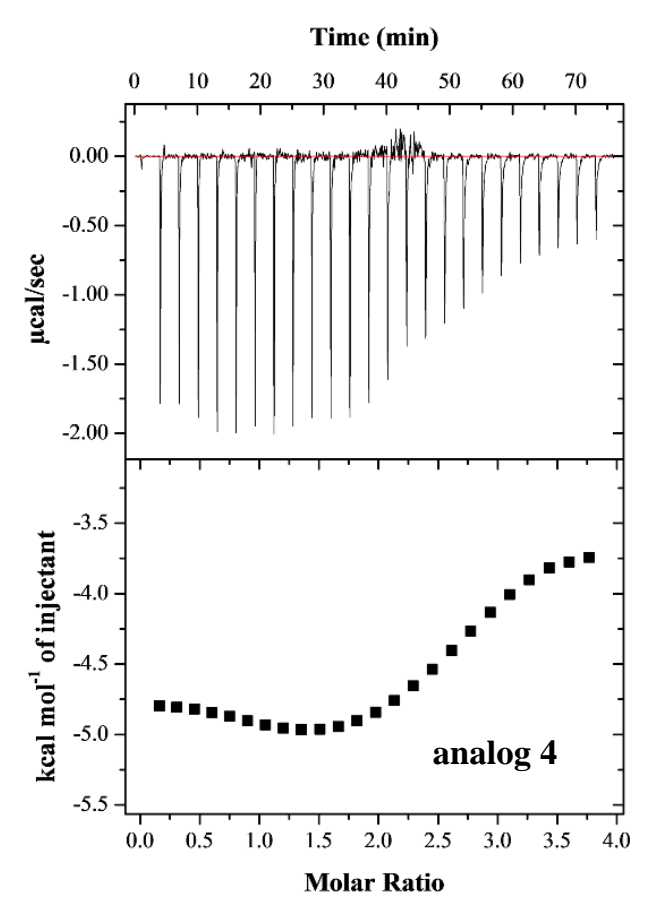

(F)

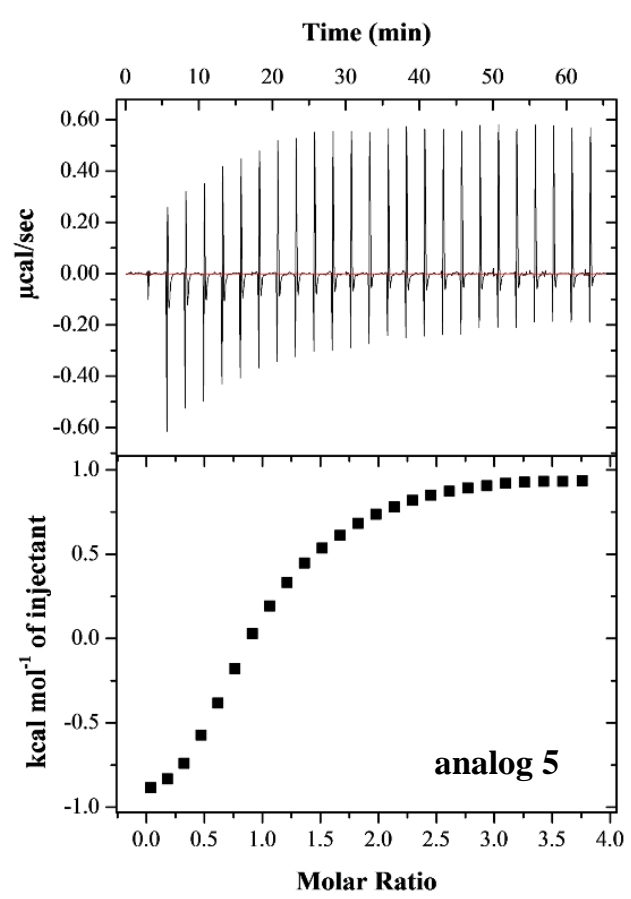

Supplementary Figure S6: Binding profiles of $M t b$ PanD with POA and its analogs. ITC profiles for WT Mtb PanD with $2 \mathrm{mM}$ of analog $\mathbf{4}(E)$ or $-\mathbf{5}(F)$. 


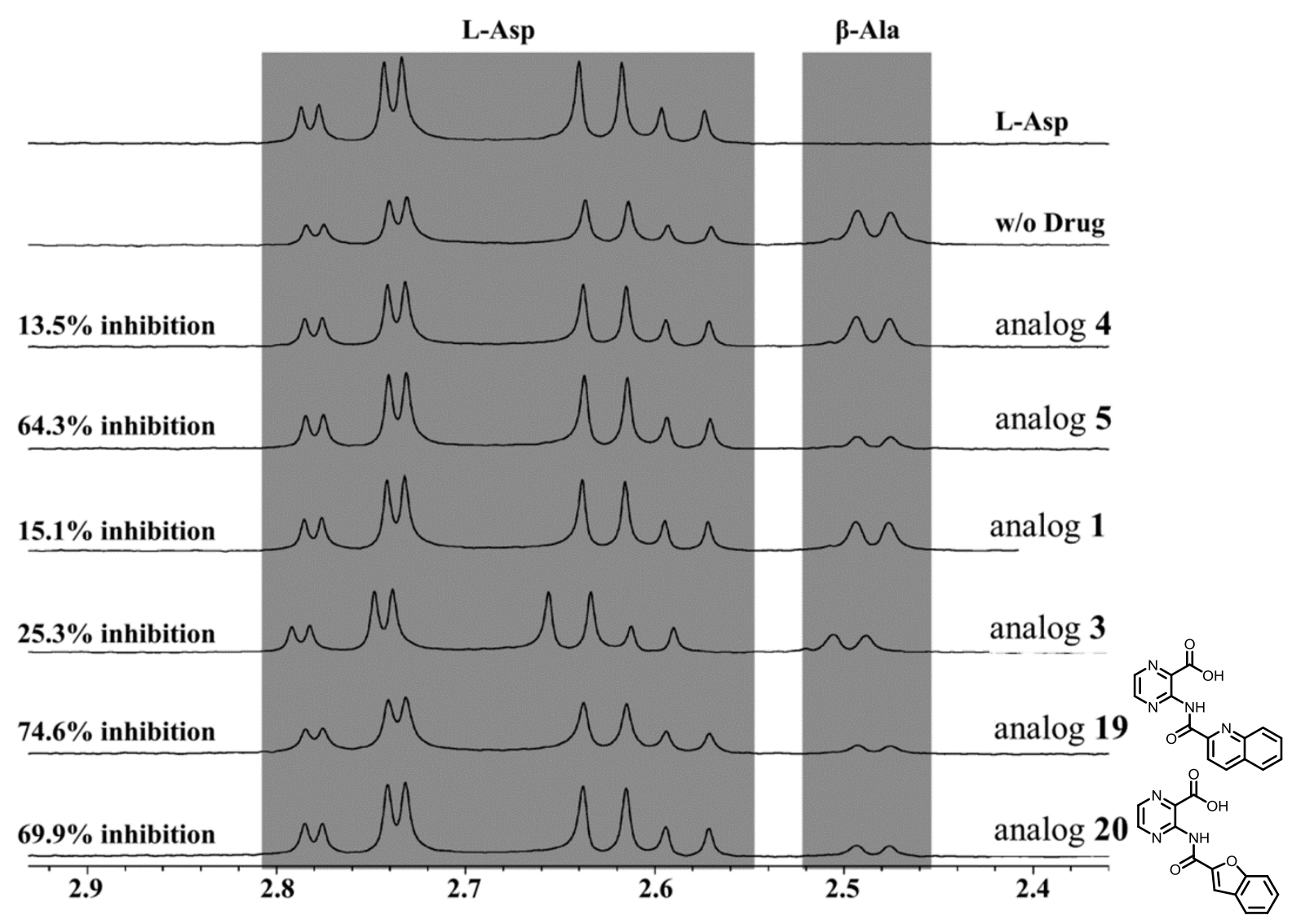

Supplementary Figure S7: Inhibitory effect of analogs 1, 3, 4, 5, 19 and 20 at a concentration of $200 \mu \mathrm{M}$ on $M t b$ PanD enzyme activity measured by ${ }^{1} \mathrm{H}-1 \mathrm{D}-\mathrm{NMR}$. The inhibition rate of $M t b$ PanD activity by the respective analog after reaction is displayed in each NMR spectra. 


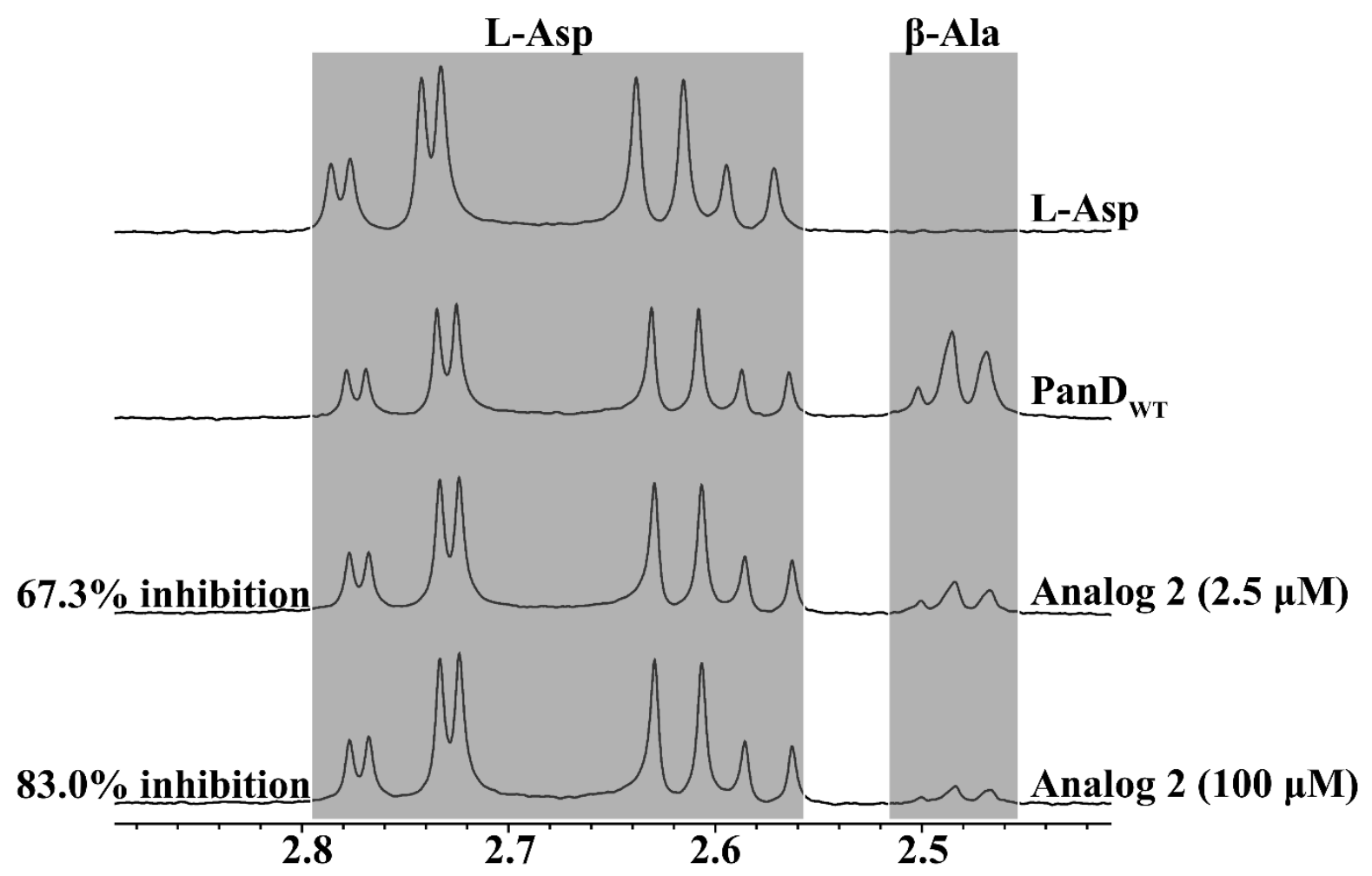

Supplementary Figure S8: Inhibitory effect of analog 2 resolved in phosphate buffer on $M t b$ PanD enzyme activity measured by ${ }^{1} \mathrm{H}-1 \mathrm{D}-\mathrm{NMR}$. Representative ${ }^{1} \mathrm{H}^{-}{ }^{1} \mathrm{D}$ NMR spectra of enzymatic conversion of L-Asp to $\beta$-Ala in the absence or presence of analog 2 (concentration of 2.5 and $100 \mu \mathrm{M}$ ), which was dissolved in $20 \mathrm{mM}$ phosphate buffer, $\mathrm{pH}$ 6.5, reflecting that analog $\mathbf{2}$ is a potent inhibitor in organic and water solvent. Inhibition rate of enzyme activity after reaction is displayed. Analog 2 was incubated for 40 min at 298K. 
Supplementary Table 1: Dissociation constant values of POA on binding with respective Nand N-terminal Mtb PanD mutants. $100 \mu \mathrm{M}$ protein were titrated with $2 \mathrm{mM}$ of POA for all the experiments.

\begin{tabular}{ll} 
PanD mutant & $\boldsymbol{K}_{\boldsymbol{D}}(\mathbf{i n} \boldsymbol{\mu M})$ \\
\hline K7A & $123 \pm 0.3$ \\
K9A & No binding \\
K11A & No binding \\
K9AH11AH21A & No binding \\
K9AH11AC17AH21A & No binding \\
T16A & No binding \\
C17A & $184 \pm 0.8$ \\
D31E & $18 \pm 0.2$ \\
H119N & $37 \pm 0.5$ \\
E126A & N.D. ${ }^{\text {a }}$ \\
L132P & $23 \pm 0.6$ \\
P134S & $10 \pm 0.6$
\end{tabular}

${ }^{\mathrm{a}}$ Not determined 
Supplementary Table 2: Primer sequences used for creating mutants of Mtb PanD

\begin{tabular}{|c|c|c|}
\hline Mutant & Name & Sequence \\
\hline \multirow[t]{2}{*}{ K7A } & Forward & 5' - ATG CTG GCT TCG AAG ATC CAC CGC GCC - 3' \\
\hline & Reverse & 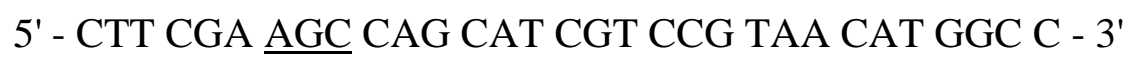 \\
\hline
\end{tabular}

K9A Forward 5' - AAG TCG GCA ATC CAC CGC GCC ACG - 3'

Reverse $\quad 5^{\prime}-$ GTG GAT TGC CGA CTT CAG CAT CGT CCG T - 3’

H11A Forward 5' - AGT CGA AGA TCG CAC GCG CCA CGG TGA CCT- 3'

Reverse $\quad$ 5' - CGC GTG CGA TCT TCG ACT TCA GCA TCG TCC GT - 3’'

T16A Forward 5' - ACG GTG GCA TGC GCC GAC CTG CAC TAC GTC - 3’

Reverse $\quad$ 5' - GAC GTA GTG CAG GTC GGC GCA TGC CAC CGT - 3’

T16S Forward 5' - ACG GTG AGT TGC GCC GAC CTG CAC TAC GTC - 3'

Reverse $\quad$ 5' - GAC GTA GTG CAG GTC GGC GCA ACT CAC CGT - 3’

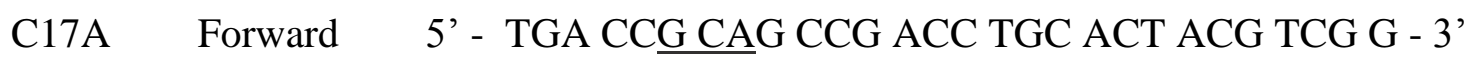

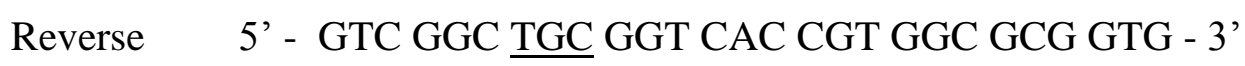

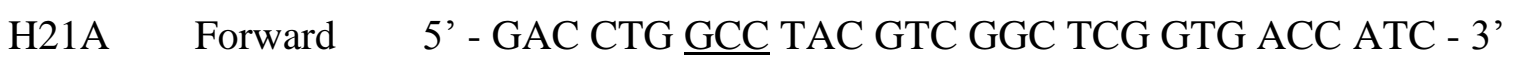

Reverse $\quad 5$ ' - GAC GTA GGC CAG GTC GGC GCA GGT CAC C - 3'

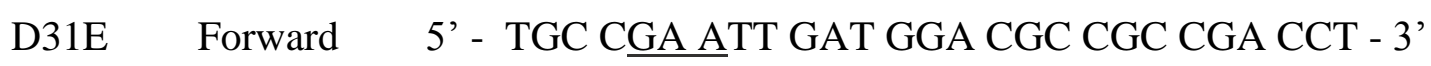

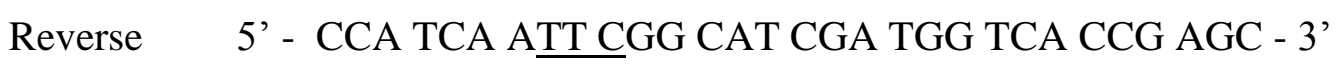

H119N Forward 5' - CAA ATG CCG GAT CGT TGC CCA TGT CGA TCG G - 3'

Reverse 5' - CCG ATC GAC ATG GGC AAC GAT CCG GCA TTT G - 3’ 
E126A Forward 5' - CTC GCC CGC GTT TGC GGG CAC AAA TGC - 3’

Reverse $\quad 5$ ' - GCA TTT GTG CCC GCA AAC GCG GGC GAG - 3’

L132P Forward 5' - GCC GGG GGT CTG GCA GCT CGC CC - 3'

Reverse 5' - GGG CGA GCT GCC AGA CCC CCG GC - 3’

P134S Forward 5' - CAC ACC GAG CCG GCT GTC TAG CAG CTC G - 3'

Reverse $\quad$ 5' - CGA GCT GCT AGA CAG CCG GCT CGG TGT G - 3'

K9A/ Forward 5' - AGT CGG CAA TCG CAC GCG CCA CGG TGA CCT - 3'

H11A Reverse 5' - CGT GCG ATT GCC GAC TTC AGC ATC GTC CGT - 3'

K9A/ Forward 5' - GAC CTG GCC TAC GTC GGC TCG GTG ACC ATC - 3'

H11A/ Reverse 5' - GGT GAC CTG CGC CGA CCT GGC CTA CGT C - 3'

K9A/ Forward 5' - GTG ACC GCC GCC GAC CTG GCC TAC GTC GGC T - 3'

H11A/ Reverse 5' - GTC GGC GGC GGT CAC CGT GGC GCG TGC GAT T - 3,

H21A

PanD $_{25-}$ Forward 5' - TTT CCA TGG CTT CGG TGA CCA TCG A - 3'

139

Reverse 5' - GTA GAG CTC CTA TCC CAC ACC GAG CC - 3'

Codons of respective residues that have been mutated have been underlined. 
Supplementary Table 3B: MALDI TOF data of upper band of the twin band about $15 \mathrm{kDa}$

\begin{tabular}{|c|c|c|c|c|c|c|c|c|c|c|}
\hline \multicolumn{6}{|l|}{ Protein name } & Accession No. & \multicolumn{4}{|l|}{ Protein MW } \\
\hline \multicolumn{11}{|c|}{ Aspartate 1-decarboxylase } \\
\hline \multicolumn{6}{|c|}{$\begin{array}{l}\text { OS=Mycobacterium tuberculosis (strain ATCC } 25618 / \\
\text { H37Rv) OX=83332 }\end{array}$} & sp|P9WIL3|PAND_MYCTU & 14932.6 & & & \\
\hline Calc. Mass & Obsrv. Mass & $\pm \mathrm{Da}$ & $\pm \mathbf{p p m}$ & $\begin{array}{l}\text { Start } \\
\text { seq. }\end{array}$ & End seq. & Sequence & ION score & C. I. $\%$ & Modification & $\begin{array}{l}\text { Rank Result } \\
\text { Type }\end{array}$ \\
\hline 891.4795 & 891.4845 & 0.005 & 6 & 98 & 104 & ARTYQPR & & & & Mascot \\
\hline 891.4795 & 891.4845 & 0.005 & 6 & 98 & 104 & ARTYQPR & & & & Mascot \\
\hline 924.5005 & 924.4393 & -0.0612 & -66 & 1 & 7 & MLRTMLK & & & Oxidation $(\mathrm{M})[1,5]$ & Mascot \\
\hline 1122.6154 & 1122.6306 & 0.0152 & 14 & 55 & 64 & LVTYAITGER & 59 & 99.998 & & Mascot \\
\hline 1122.6154 & 1122.6306 & 0.0152 & 14 & 55 & 64 & LVTYAITGER & & & & Mascot \\
\hline 3303.7153 & 3303.7822 & 0.0669 & 20 & 65 & 97 & GSGVIGINGAAAHLVHPGDLVILIAYATMDDAR & & & Oxidation (M)[29] & Mascot \\
\hline 3303.7153 & 3303.7822 & 0.0669 & 20 & 65 & 97 & GSGVIGINGAAAHLVHPGDLVILIAYATMDDAR & & & Oxidation (M)[29] & Mascot \\
\hline 3455.6938 & 3455.739 & 0.0452 & 13 & 105 & 135 & IVFVDAYNKPIDMGHDPAFVPENAGELLDPR & & & Oxidation (M)[13] & Mascot \\
\hline
\end{tabular}

The MALDI TOF/TOF experiments were carried out on an ABI 4800 Proteomics Analyzer MALDI TOF/TOF mass spectrometer (Applied

Biosystems) using the established protocols of the Proteomics Department, School of Biological Sciences, Nanyang Technological University

Singapore (3). 
Supplementary Table 3B: MALDI TOF data of lower band of the twin band about 15 kDa

\begin{tabular}{|c|c|c|c|c|c|c|c|c|c|c|}
\hline \multicolumn{6}{|c|}{ Protein name } & Accession No. & \multicolumn{4}{|l|}{ Protein MW } \\
\hline \multicolumn{11}{|c|}{ Aspartate 1-decarboxylase } \\
\hline \multicolumn{6}{|c|}{$\begin{array}{l}\text { OS=Mycobacterium tuberculosis (strain ATCC } \\
25618 \text { / H37Rv) OX=83332 }\end{array}$} & sp|P9WIL3|PAND_MYCTU & 14932.6 & & & \\
\hline Calc. Mass & Obsrv. Mass & $\pm \mathrm{Da}$ & $\begin{array}{c} \pm \\
\text { ppm }\end{array}$ & $\begin{array}{l}\text { Start } \\
\text { seq. }\end{array}$ & End seq. & Sequence & ION score & C. I. \% & Modification & Rank Result Type \\
\hline 891.4795 & 891.4954 & 0.0159 & 18 & 98 & 104 & ARTYQPR & & & & Mascot \\
\hline 891.4795 & 891.4954 & 0.0159 & 18 & 98 & 104 & ARTYQPR & & & & Mascot \\
\hline 1122.6154 & 1122.6415 & 0.0261 & 23 & 55 & 64 & LVTYAITGER & 59 & 99.998 & & Mascot \\
\hline 1122.6154 & 1122.6415 & 0.0261 & 23 & 55 & 64 & LVTYAITGER & & & & Mascot \\
\hline 3303.7153 & 3303.793 & 0.0777 & 24 & 65 & 97 & GSGVIGINGAAAHLVHPGDLVILIAYATMDDAR & & & Oxidation (M)[29] & Mascot \\
\hline 3303.7153 & 3303.793 & 0.0777 & 24 & 65 & 97 & GSGVIGINGAAAHLVHPGDLVILIAYATMDDAR & & & Oxidation (M)[29] & Mascot \\
\hline 3455.6938 & 3455.7668 & 0.073 & 21 & 105 & 135 & IVFVDAYNKPIDMGHDPAFVPENAGELLDPR & & & Oxidation (M)[13] & Mascot \\
\hline
\end{tabular}

The MALDI TOF/TOF experiments were carried out on an ABI 4800 Proteomics Analyzer MALDI TOF/TOF mass spectrometer (Applied Biosystems) using the established protocols of the Proteomics Department, School of Biological Sciences, Nanyang Technological University Singapore (3). 


\section{Part II: Synthetic Chemistry}

\section{$\underline{\text { General Remarks }}$}

Chemical reagents and solvents were purchased from Alfa Aesar, Fisher Scientific, SigmaAldrich, TCI America or Oakwood Chemical and used without additional purification. Dichloromethane (DCM), methanol (MeOH), and tetrahydrofuran (THF) were dispensed under Argon (Ar) using an Inert solvent purification system. 1,4-dioxanes was purchased from Fisher Scientific and degassed via sonification under vacuum for Suzuki-Miyaura reactions. Ethyl acetate (EtOAc) and hexanes were purchased from Fisher Scientific and used without additional purification. Anhydrous reactions were performed under inert gas (Ar or $\mathrm{N}_{2}$ ) in flame-dried glassware. Thin-layer chromatography (TLC) was performed on TLC silica gel 60F254 plates from EMD Chemical Inc. and visualized using UV light or $\mathrm{I}_{2}$ staining. Reaction purification was performed via column chromatography employing a Teledyne ISCO RF-200 CombiFlash system with the indicated solvent gradient; flash column silica gel cartridges were used for purifying intermediates, while RediSep ${ }^{\circledR}$ Rf Gold ${ }^{\circledR}$ Cyano cartridges (Teledyne ISCO) were used for purification of final carboxylic acid products. Percent purity analysis was performed using analytical high pressure liquid chromatography (HPLC) with a reversed-phase XSelect ${ }^{\circledR}$ CSH $5 \mu \mathrm{m}$ C-18 4.6 x 150 mm LC column, with detection at $250 \mathrm{~nm}$. A gradient method of $5 \%$ to $95 \%$ acetonitrile $(\mathrm{MeCN})$ in water over 15 min at a flow rate of $1.0 \mathrm{ml} / \mathrm{min}$ was employed; both solvents were treated with either $0.1 \%$ formic acid (most analyses) or $20 \mathrm{mM}$ ammonium acetate (analogs 9 and 20 only) to assist resolution. Nuclear magnetic resonance (NMR) spectroscopy was performed using a 500 or $600 \mathrm{MHz}$ spectrometer (Bruker) and calibrating to known chemical shifts for residual solvent peaks. High resolution mass spectrometry (HRMS) analysis was performed using an automated 6230 TOF LC/MS system (Agilent).

\section{POAAnalogue Syntheses}

Esterification of 3-chloro and 3-aminopyrazine

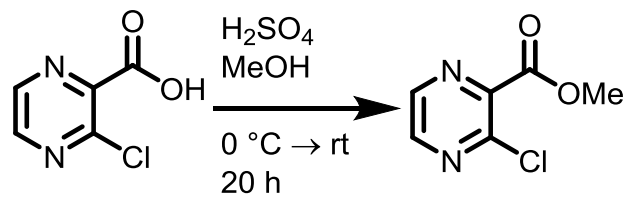

3-chloropyrazinoic acid (3 g, $18.9 \mathrm{mmol})$ was dissolved in $\mathrm{MeOH}(18.9 \mathrm{ml}, 1 \mathrm{M})$ and cooled to $0{ }^{\circ} \mathrm{C}$. Concentrated sulfuric acid $(3.8 \mathrm{ml}, 70 \mathrm{mmol})$ was added slowly, and the mixture was gradually warmed to room temperature (rt) with stirring overnight. After $20 \mathrm{~h}$, the reaction was neutralized to $\mathrm{pH} \sim 8$ with saturated aqueous sodium bicarbonate solution, then extracted into several portions of EtOAc (3x $50 \mathrm{ml})$. The organic layers were combined, washed with brine, dried with $\mathrm{MgSO}_{4}$ and condensed onto silica gel. The crude reaction was purified by flash column chromatography (hexanes/ EtOAc, 0\% to 75\% EtOAc over $20 \mathrm{~min}$ ) and the major peak was collected and condensed, yielding the product as a thick yellow oil which crystallized into a white solid upon cooling (1.65 g, 51\% yield). The product was characterized by NMR and matched previously reported spectral data for the compound (4). 


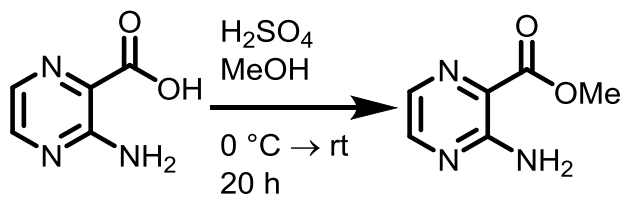

An identical procedure was employed for the preparation of 3-methylpyrazine carboxylate, yielding $1.53 \mathrm{~g}$ of crude product from $3 \mathrm{~g}$ of the starting acid (46\% yield) as a brown solid, which matched previously reported spectral data and was taken on without additional purification (5).

General amidation procedure<smiles>[R]C(=O)Nc1ncc(C([R])C(=O)Cl)nc1C(=O)OC</smiles>

Methyl 3-amino-2-pyrazinecarboxylate (1 equiv) was dissolved in DCM (0.5 M) in a two dram vial equipped with a pressure release cap. Acid chloride (2.0 equiv) was added slowly and the reaction mixture was stirred at $\mathrm{rt}\left(50{ }^{\circ} \mathrm{C}\right.$ for hindered acid chlorides noted) and monitored for completion by TLC (50-75\% ETOAC/hexanes). Following consumption of starting material, the crude mixture was diluted with DCM, washed with saturated aqueous $\mathrm{Na}_{2} \mathrm{CO}_{3}$ solution and brine, dried with $\mathrm{MgSO}_{4}$ and condensed onto silica gel. Purification was performed using column chromatography (ETOAC/hexanes, gradient method) and the major peak was collected.

Methyl 3-(1-naphthamido)pyrazine-2-carboxylate (2-OMe). The title compound was obtained from 1-naphthoyl chloride in $31 \%$ yield $\left(50{ }^{\circ} \mathrm{C}, 72 \mathrm{~h}\right.$ reaction time) as a yelloworange solid. $R_{f}=0.15$ (50:50 EtOAc:hexanes); ${ }^{1} \mathrm{H}$ NMR (DMSO- $\left.d_{6}\right) \delta 11.61(\mathrm{~s}, 1 \mathrm{H}), 8.73$ $(\mathrm{d}, \mathrm{J}=2.4 \mathrm{~Hz}, 1 \mathrm{H}), 8.59(\mathrm{~d}, \mathrm{~J}=2.4 \mathrm{~Hz}, 1 \mathrm{H}), 8.31-8.25(\mathrm{~m}, 1 \mathrm{H}), 8.13(\mathrm{~d}, \mathrm{~J}=8.2 \mathrm{~Hz}, 1 \mathrm{H})$, $8.07-8.01(\mathrm{~m}, 1 \mathrm{H}), 7.81(\mathrm{dd}, \mathrm{J}=7.1,1.2 \mathrm{~Hz}, 1 \mathrm{H}), 7.68-7.57(\mathrm{~m}, 3 \mathrm{H}), 3.84(\mathrm{~s}, 3 \mathrm{H}) ;{ }^{13} \mathrm{C}$ NMR (DMSO- $\left.d_{6}\right) \delta 168.07,165.06,145.35,140.03,138.66,133.15,132.48,131.10,129.75$, $128.39,127.18,126.47,126.43,124.98,52.51$.

Methyl 3-benzaamidopyrazine-2-carboxylate (5-OMe). The title compound was obtained from benzoyl chloride in $29 \%$ yield ( $\mathrm{rt}, 24 \mathrm{~h}$ reaction time) as an orange solid. $R_{f}=0.10$ (50:50 EtOAc:hexanes); ${ }^{1} \mathrm{H}$ NMR (DMSO- $\left.d_{6}\right) \delta 11.39(\mathrm{~s}, 1 \mathrm{H}), 8.73(\mathrm{~d}, \mathrm{~J}=2.3 \mathrm{~Hz}, 1 \mathrm{H}), 8.57$ $(\mathrm{d}, \mathrm{J}=2.4 \mathrm{~Hz}, 1 \mathrm{H}), 8.05-7.98(\mathrm{~m}, 2 \mathrm{H}), 7.69-7.61(\mathrm{~m}, 1 \mathrm{H}), 7.56(\mathrm{dd}, \mathrm{J}=8.3,7.0 \mathrm{~Hz}, 2 \mathrm{H})$, $3.76(\mathrm{~s}, 3 \mathrm{H}) ;{ }^{13} \mathrm{C}$ NMR (DMSO- $\left.d_{6}\right) \delta 166.15,164.88,145.89,145.22,139.85,138.47,132.89$, $132.54,128.57,128.05,52.37$.

Methyl 3-propionamidopyrazine-2-carboxylate (7-OMe). The title compound was obtained from propionyl chloride in $77 \%$ yield ( $\mathrm{rt}, 24 \mathrm{~h}$ reaction time) as a crystalline yellow solid. $R_{f}=0.20$ (75:25 EtOAc:hexanes); ${ }^{1} \mathrm{H}$ NMR (DMSO- $\left.d_{6}\right) \delta 10.90(\mathrm{~s}, 1 \mathrm{H}), 8.62$ (d, J = $2.4 \mathrm{~Hz}, 1 \mathrm{H}), 8.47(\mathrm{~d}, \mathrm{~J}=2.3 \mathrm{~Hz}, 1 \mathrm{H}), 3.76$ (s, 3H), 2.39 (q, J = 7.6 Hz, 2H), 1.06 (t, J = 7.5 
$\mathrm{Hz}, 3 \mathrm{H}) ;{ }^{13} \mathrm{C}$ NMR (DMSO- $\left.d_{6}\right) \delta 172.90,164.83,145.03,144.93,139.33,138.01,52.14$, 28.76, 9.20.

Methyl 3-(2-naphthamido)pyrazine-2-carboxylate (12-OMe). The title compound was obtained from 2-naphthoyl chloride in $64 \%$ yield ( $\mathrm{rt}, 48 \mathrm{~h}$ reaction time) as a red solid. $R_{f}=$ 0.25 (75:25 EtOAc:hexanes); ${ }^{1} \mathrm{H}$ NMR (DMSO- $\left.d_{6}\right) \delta 11.86(\mathrm{~s}, 1 \mathrm{H}), 8.75(\mathrm{~d}, \mathrm{~J}=2.3 \mathrm{~Hz}, 1 \mathrm{H})$, $8.60(\mathrm{~d}, \mathrm{~J}=1.7 \mathrm{~Hz}, 1 \mathrm{H}), 8.46(\mathrm{~d}, \mathrm{~J}=2.3 \mathrm{~Hz}, 1 \mathrm{H}), 8.11(\mathrm{dd}, \mathrm{J}=8.6,1.9 \mathrm{~Hz}, 1 \mathrm{H}), 8.05(\mathrm{~d}, \mathrm{~J}=$ $7.4 \mathrm{~Hz}, 1 \mathrm{H}), 8.00(\mathrm{~d}, \mathrm{~J}=8.6 \mathrm{~Hz}, 1 \mathrm{H}), 7.93$ (d, J = 7.6 Hz, 1H), $7.67-7.55$ (m, 2H), 4.12 (s, $3 \mathrm{H}) ;{ }^{13} \mathrm{C}$ NMR (DMSO- $\left.d_{6}\right) \delta 167.22,164.79,150.55,147.38,138.63,135.49,132.82$, $131.39,129.61,129.11,128.96,128.92,128.52$, 127.97, 127.13, 123.83, 53.97.

Methyl 3-(quinoline-2-carboxamido)pyrazine-2-carboxylate (19-OMe). The title compound was obtained from quinaldoyl chloride in $58 \%$ yield $\left(50{ }^{\circ} \mathrm{C}, 72 \mathrm{~h}\right.$ reaction time $)$ as a reddish solid. $R_{f}=0.20$ (75:25 EtOAc:hexanes); ${ }^{1} \mathrm{H}$ NMR (DMSO- $\left.d_{6}\right) \delta 13.18(\mathrm{~s}, 1 \mathrm{H}), 8.75$ $(\mathrm{d}, \mathrm{J}=2.3 \mathrm{~Hz}, 1 \mathrm{H}), 8.48(\mathrm{~d}, \mathrm{~J}=2.2 \mathrm{~Hz}, 1 \mathrm{H}), 8.41(\mathrm{q}, \mathrm{J}=8.5 \mathrm{~Hz}, 2 \mathrm{H}), 8.35(\mathrm{~d}, \mathrm{~J}=8.5 \mathrm{~Hz}$, $1 \mathrm{H}), 7.94(\mathrm{~d}, \mathrm{~J}=8.2 \mathrm{~Hz}, 1 \mathrm{H}), 7.85$ (ddd, J = 8.4, 6.8, $1.5 \mathrm{~Hz}, 1 \mathrm{H}), 7.69$ (ddd, J = 8.1, 6.9, 1.2 $\mathrm{Hz}, 1 \mathrm{H}), 4.17(\mathrm{~s}, 3 \mathrm{H}) ;{ }^{13} \mathrm{C}$ NMR (DMSO- $\left.d_{6}\right) \delta 166.14,162.86,149.64,149.30,146.93$, $146.65,138.68,138.05,130.57,130.41,130.24,129.85,128.78,127.88,119.27,53.75$.

Methyl 3-(benzofuran-2-carboxamido)pyrazine-2-carboxylate (20-OMe). The title compound was obtained from benzofuran-2-carbonyl chloride in $56 \%$ yield $\left(50{ }^{\circ} \mathrm{C}, 72 \mathrm{~h}\right.$ reaction time) as a yellow-brown solid. $R_{f}=0.20$ (75:25 EtOAc:hexanes); ${ }^{1} \mathrm{H}$ NMR (DMSO$\left.d_{6}\right) \delta 12.49(\mathrm{~s}, 1 \mathrm{H}), 9.57(\mathrm{~d}, \mathrm{~J}=2.4 \mathrm{~Hz}, 1 \mathrm{H}), 9.41(\mathrm{~d}, \mathrm{~J}=2.4 \mathrm{~Hz}, 1 \mathrm{H}), 8.78(\mathrm{~d}, \mathrm{~J}=0.9 \mathrm{~Hz}$, $1 \mathrm{H}), 8.67(\mathrm{dd}, \mathrm{J}=7.8,1.1 \mathrm{~Hz}, 1 \mathrm{H}), 8.56(\mathrm{~d}, \mathrm{~J}=8.4 \mathrm{~Hz}, 1 \mathrm{H}), 8.36(\mathrm{ddd}, \mathrm{J}=8.4,7.2,1.3 \mathrm{~Hz}$, $1 \mathrm{H}), 8.21(\mathrm{t}, \mathrm{J}=7.5 \mathrm{~Hz}, 1 \mathrm{H}), 4.64(\mathrm{~s}, 3 \mathrm{H}) ;{ }^{13} \mathrm{C}$ NMR (DMSO- $\left.d_{6}\right) \delta 164.96,156.96,154.70$, $147.33,145.55,145.34,140.16,137.47,127.82,126.89,124.07,123.29,112.30,112.08$, 52.57 .

General Amination Procedure

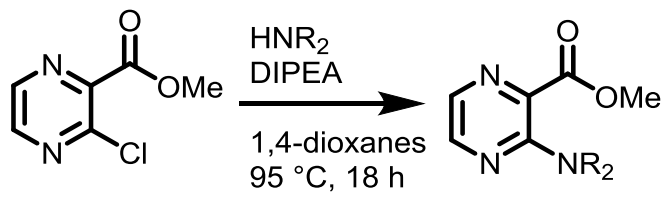

Methyl 3-chloro-2-pyrazinecarboxylate (1 equiv), diisopropylethylamine (3 equiv) and amine ( 2 equiv) were combined in a sealed round bottom flask, dissolved in 1,4-dioxanes $(0.2 \mathrm{M})$, heated to $95{ }^{\circ} \mathrm{C}$ and stirred overnight. The reaction was monitored by TLC $(50 \%$ EtOAc/hexanes) for consumption of starting material. Upon completion, the crude reaction mixture was diluted with DCM and condensed onto celite. The dry load was purified by column chromatography (EtOAc/hexanes, gradient method) and the major peak was collected.

Methyl 3-(phenylamino)pyrazine-2-carboxylate (3-OMe). The title compound was obtained from aniline in $63 \%$ yield as a white solid. $R_{f}=0.38$ (50:50 EtOAc:hexanes); ${ }^{1} \mathrm{H}$ $\operatorname{NMR}\left(\mathrm{CDCl}_{3}\right) \delta 8.81(\mathrm{~d}, J=1.5 \mathrm{~Hz}, 1 \mathrm{H}), 8.16(\mathrm{~d}, J=1.5 \mathrm{~Hz}, 1 \mathrm{H}), 7.43(\mathrm{~d}, J=7.9 \mathrm{~Hz}, 2 \mathrm{H})$, 
$7.33(\mathrm{t}, J=7.9 \mathrm{~Hz}, 2 \mathrm{H}), 7.12(\mathrm{dd}, J=8.1,6.6 \mathrm{~Hz}, 1 \mathrm{H}), 7.01(\mathrm{~d}, J=4.5 \mathrm{~Hz}, 1 \mathrm{H}), 3.91(\mathrm{~s}, 3 \mathrm{H})$;

${ }^{13} \mathrm{C} \mathrm{NMR}\left(\mathrm{CDCl}_{3}\right) \delta 164.9,153.6,145.4,137.8,133.3,132.1,129.6,125.0,121.2,52.4$.

Methyl 3-(ethylamino)-2-pyrazine-2-carboxylate (8-OMe).

The title compound was obtained from ethylamine in $40 \%$ yield as a yellow solid. $R_{f}=0.18$ (25:75 EtOAc:hexanes); ${ }^{1} \mathrm{H}$ NMR $\left(\mathrm{CDCl}_{3}\right) \delta 8.17(\mathrm{~d}, J=2.2 \mathrm{~Hz}, 1 \mathrm{H}), 7.79(\mathrm{~d}, J=2.2 \mathrm{~Hz}$, $1 \mathrm{H}), 3.90(\mathrm{~s}, 3 \mathrm{H}), 3.47(\mathrm{qd}, J=7.1,5.1 \mathrm{~Hz}, 2 \mathrm{H}), 1.22(\mathrm{~d}, J=7.2 \mathrm{~Hz}, 3 \mathrm{H}) ;{ }^{13} \mathrm{C} \mathrm{NMR}\left(\mathrm{CDCl}_{3}\right)$ $\delta 167.3,155.4,147.5,131.2,52.7,35.6,14.7$.

Methyl 3-(isobutylamino)pyrazine-2-carboxylate (9-OMe). The title compound was obtained from isobutylamine in 64\% yield as a yellow oil. $R_{f}=0.35$ (35:65 EtOAc:hexanes); ${ }^{1} \mathrm{H} \mathrm{NMR}\left(\mathrm{CDCl}_{3}\right) \delta 8.21(\mathrm{~d}, \mathrm{~J}=2.2 \mathrm{~Hz}, 1 \mathrm{H}), 8.08(\mathrm{~s}, 1 \mathrm{H}), 7.84(\mathrm{~d}, \mathrm{~J}=2.2 \mathrm{~Hz}, 1 \mathrm{H}), 3.97(\mathrm{~s}$, $3 \mathrm{H}), 3.34(\mathrm{dd}, \mathrm{J}=6.9,5.6 \mathrm{~Hz}, 2 \mathrm{H}), 1.98-1.88(\mathrm{~m}, 1 \mathrm{H}), 0.99(\mathrm{~d}, \mathrm{~J}=6.7 \mathrm{~Hz}, 6 \mathrm{H}) ;{ }^{13} \mathrm{C} \mathrm{NMR}$ (DMSO- $\left.d_{6}\right) \delta 166.85,154.85,147.38,131.13,123.75,52.24,52.10,47.32,27.53,20.03$.

Methyl 3-(cyclobutylamino)pyrazine-2-carboxylate (10-OMe). The title compound was obtained from cyclobutylamine $67 \%$ yield as an off-white solid. $R_{f}=0.55$ (50:50 EtOAc:hexanes); ${ }^{1} \mathrm{H}$ NMR $\left(\mathrm{CDCl}_{3}\right) \delta 8.15(\mathrm{~d}, J=2.3 \mathrm{~Hz}, 1 \mathrm{H}), 7.80(\mathrm{~d}, J=2.3 \mathrm{~Hz}, 1 \mathrm{H})$, 4.54-4.43 (m, 1H), 3.91 (s, 3H), 2.43-2.33 (m, 2H), 1.97-1.85 (m, 2H), 1.83-1.67 (m, 2H); ${ }^{13} \mathrm{C} \mathrm{NMR}\left(\mathrm{CDCl}_{3}\right) \delta 167.3,154.5,147.5,131.5,124.0,52.7,45.9,31.3,15.4$.

Methyl 3-(diethylamino)pyrazine-2-carboxylate(13-OMe). The title compound was obtained from diethylamine in 59\% yield as a yellow oil. $R_{f}=0.70$ (75:25 EtOAc:hexanes); ${ }^{1} \mathrm{H}$ NMR $\left(\mathrm{CDCl}_{3}\right) \delta 8.11(\mathrm{~d}, \mathrm{~J}=2.1 \mathrm{~Hz}, 1 \mathrm{H}), 7.85(\mathrm{~d}, \mathrm{~J}=2.2 \mathrm{~Hz}, 1 \mathrm{H}), 3.95(\mathrm{~s}, 3 \mathrm{H}), 3.45$ (q, J $=7.1 \mathrm{~Hz}, 4 \mathrm{H}), 1.19(\mathrm{t}, \mathrm{J}=7.1 \mathrm{~Hz}, 6 \mathrm{H}) ;{ }^{13} \mathrm{C} \mathrm{NMR}\left(\mathrm{CDCl}_{3}\right) \delta 167.23,153.17,143.18,131.16$, $130.08,52.94,43.85,12.58$.

Methyl 3-(benzylamino)pyrazine-2-carboxylate(17-OMe): The title compound was obtained from benzylamine in $86 \%$ yield as an off-white solid. $R_{f}=0.51$ (50:50 EtOAc:hexanes); ${ }^{1} \mathrm{H}$ NMR $\left(\mathrm{CDCl}_{3}\right) \delta 8.37(\mathrm{~s}, 1 \mathrm{H}), 8.28(\mathrm{~d}, J=2.9 \mathrm{~Hz}, 1 \mathrm{H}), 8.04-7.90(\mathrm{~m}$, $1 \mathrm{H}), 7.38(\mathrm{t}, J=5.5 \mathrm{~Hz}, 4 \mathrm{H}), 7.32-7.22(\mathrm{~m}, 2 \mathrm{H}), 4.77(\mathrm{t}, J=4.6 \mathrm{~Hz}, 2 \mathrm{H}), 4.00(\mathrm{~d}, J=3.7 \mathrm{~Hz}$, $3 \mathrm{H}) ;{ }^{13} \mathrm{C} \mathrm{NMR}\left(\mathrm{CDCl}_{3}\right) \delta 167.3,155.3,147.5,138.6,131.8,128.7,127.5,124.4,52.8,44.6$.

Methyl 3-(cyclopentylamino)pyrazine-2-carboxylate(18-OMe): The title compound was obtained from cyclopentylamine in $96 \%$ yield as a yellow oil. $R_{f}=0.60$ (50:50 EtOAc:hexane); ${ }^{1} \mathrm{H}$ NMR $\left(\mathrm{CDCl}_{3}\right) \delta 8.16(\mathrm{~d}, J=2.1 \mathrm{~Hz}, 1 \mathrm{H}), 7.94(\mathrm{~s}, 1 \mathrm{H}), 7.78(\mathrm{t}, J=1.7$ $\mathrm{Hz}, 1 \mathrm{H}), 4.33(\mathrm{~h}, J=6.7 \mathrm{~Hz}, 1 \mathrm{H}), 3.90(\mathrm{~d}, J=1.3 \mathrm{~Hz}, 3 \mathrm{H}), 2.01(\mathrm{dq}, J=12.7,6.6 \mathrm{~Hz}, 2 \mathrm{H})$, $1.77-1.65(\mathrm{~m}, 2 \mathrm{H}), 1.65-1.52(\mathrm{~m}, 2 \mathrm{H}), 1.46(\mathrm{dq}, J=14.0,7.6,7.1 \mathrm{~Hz}, 2 \mathrm{H}) ;{ }^{13} \mathrm{C} \mathrm{NMR}$ $\left(\mathrm{CDCl}_{3}\right) \delta 167.4,155.1,147.5,131.1,124.0,52.7,52.3,33.2,23.8$. 
General Suzuki-Miyaura coupling procedure

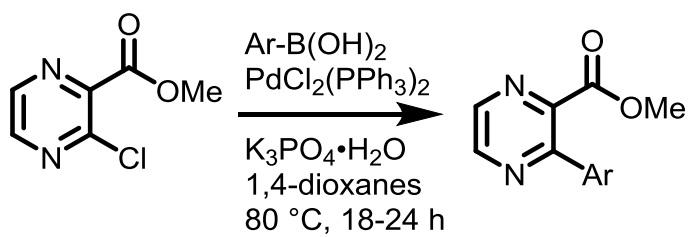

Methyl 3-chloro-2-pyrazinecarboxylate (1 equiv), aryl boronic acid (2.0 equiv), bis(triphenylphosphine)palladium(II) dichloride (10 mol\%), and potassium phosphate monohydrate ( 2.0 equiv) were combined in a 2 dram vial, which was purged under vacuum and degassed with $\mathrm{N}_{2}$. Degassed 1,4-dioxanes (sonicated under vacuum) was added under positive pressure, and vial cap was replaced with a pressure release cap. Vial was heated to 80 ${ }^{\circ} \mathrm{C}$ and stirred overnight. Reaction progress was monitored by TLC (50\% EtOAc/hexanes) to completion, then condensed in vacuo, redissolved in EtOAc and extracted from water, washing with additional EtOAc (3 portions). Combined organics were condensed onto silica and purified by column chromatography (EtOAc/hexanes).

Methyl 3-(4-fluorophenyl)pyrazine-2-carboxylate (11-OMe). The title compound was obtained from 4-fluorophenyl boronic acid in $84 \%$ yield as a red solid. $R_{f}=0.60(25: 75$ EtOAc:hexanes); ${ }^{1} \mathrm{H}$ NMR $\left(\mathrm{CDCl}_{3}\right) \delta 8.75(\mathrm{~d}, \mathrm{~J}=2.4 \mathrm{~Hz}, 1 \mathrm{H}), 8.60(\mathrm{~d}, \mathrm{~J}=2.4 \mathrm{~Hz}, 1 \mathrm{H}), 7.67$ - $7.59(\mathrm{~m}, 2 \mathrm{H}), 7.23-7.13(\mathrm{~m}, 2 \mathrm{H}), 3.87(\mathrm{~s}, 3 \mathrm{H}) ;{ }^{13} \mathrm{C} \mathrm{NMR}\left(\mathrm{CDCl}_{3}\right) ; \delta 166.71,164.95$, $152.80,145.48,144.27,141.95,133.27,130.75,130.69,115.99,115.82,53.22 ;{ }^{19} \mathrm{~F} \mathrm{NMR}$ $\left(\mathrm{CDCl}_{3}\right) \delta-111.06$.

Methyl 3-phenylpyrazine-2-carboxylic acid (14-OMe). The title compound was obtained from phenylboronic acid in $86 \%$ yield as a yellow-brown oil. Spectra and other analytical data matched previously reported values for this compound (6).

Methyl 3-(4-(Methylthio)phenyl)pyrazine-2-carboxylate (15-OMe). The title compound was obtained from 4-(methylthio)phenylboronic acid in $94 \%$ yield as a yellow oil. $R_{f}=0.55$ (25:75 EtOAc:hexanes); ${ }^{1} \mathrm{H}$ NMR $\left(\mathrm{CDCl}_{3}\right) \delta 8.74(\mathrm{~d}, \mathrm{~J}=2.4 \mathrm{~Hz}, 1 \mathrm{H}), 8.57(\mathrm{~d}, \mathrm{~J}=2.4 \mathrm{~Hz}$, $1 \mathrm{H}), 7.60-7.54(\mathrm{~m}, 2 \mathrm{H}), 7.36-7.30(\mathrm{~m}, 2 \mathrm{H}), 3.88(\mathrm{~s}, 3 \mathrm{H}), 2.53(\mathrm{~s}, 3 \mathrm{H}) ;{ }^{13} \mathrm{C} \mathrm{NMR}\left(\mathrm{CDCl}_{3}\right)$ $\delta 166.88,152.97,145.23,144.31,141.69,141.65,133.20,129.77,129.07,126.03,123.89$, $53.23,15.37$

Methyl 3-(o-Tolyl)pyrazine-2-carboxylate (16-OMe). The title compound was obtained from $o$-tolylboronic acid in $75 \%$ yield as an off-white solid. $R_{f}=0.60 \quad(25: 75$ EtOAc:hexanes); ${ }^{1} \mathrm{H} \mathrm{NMR}\left(\mathrm{CDCl}_{3}\right) \delta 8.80(\mathrm{~d}, \mathrm{~J}=2.4 \mathrm{~Hz}, 1 \mathrm{H}), 8.67(\mathrm{~d}, \mathrm{~J}=2.4 \mathrm{~Hz}, 1 \mathrm{H}), 7.36$ $(\mathrm{td}, \mathrm{J}=7.4,1.4 \mathrm{~Hz}, 1 \mathrm{H}), 7.32-7.25(\mathrm{~m}, 2 \mathrm{H}), 7.18(\mathrm{dd}, \mathrm{J}=7.6,1.3 \mathrm{~Hz}, 1 \mathrm{H}), 3.77(\mathrm{~s}, 3 \mathrm{H})$, 2.19 (s, 3H); ${ }^{13} \mathrm{C} \mathrm{NMR}\left(\mathrm{CDCl}_{3}\right) \delta 155.86,145.74,144.46,142.39,137.37,135.92,130.50$, 129.28, 128.62, 125.83, 53.08, 19.79. 
General saponification procedure

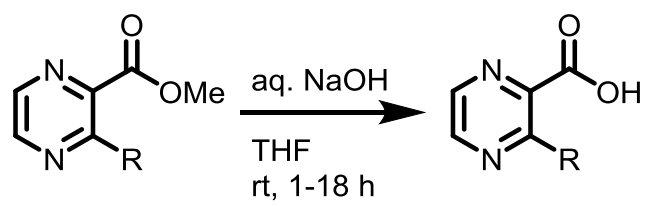

$\mathrm{R}=\mathrm{Ar}, \mathrm{NR}_{2}, \mathrm{NHCOR}$

Methyl ester (1 equiv) was dissolved in THF $(0.1 \mathrm{M})$ at $\mathrm{rt}$. Aqueous $\mathrm{NaOH}$ solution $(0.25 \mathrm{~N}, 2.5$ equiv) was added slowly over $5 \mathrm{~min}$, and the reaction was stirred at $\mathrm{rt}$ until completion as determined by TLC (25\% MeOH in DCM; $1-18 \mathrm{~h})$. The crude reaction mixture was condensed to dryness in vacuo, then resolubilized in $\mathrm{DCM} / \mathrm{MeOH}$, condensed onto celite and purified by column chromatography using a cyano-functionalized silica cartridge (Teledyne Isco) with a gradient of $0-70 \% \mathrm{MeOH}$ in DCM.

3-Amino-6-bromopyrazine-2-carboxylic acid (1). The title compound was obtained from the methyl ester in $73 \%$ yield as a yellow solid. Spectra and other analytical data matched previously reported values for this compound (7).

3-(1-Naphthamido)pyrazine-2-carboxylic acid (2). The title compound was obtained from the methyl ester in quantitative yield as an off-white solid. ${ }^{1} \mathrm{H}$ NMR (DMSO- $\left.d_{6}\right) \delta 14.81$ (s, $1 \mathrm{H}), 8.42-8.32(\mathrm{~m}, 2 \mathrm{H}), 8.27(\mathrm{~d}, \mathrm{~J}=2.5 \mathrm{~Hz}, 1 \mathrm{H}), 8.10(\mathrm{~d}, \mathrm{~J}=8.2 \mathrm{~Hz}, 1 \mathrm{H}), 8.06-7.99$ (m, $1 \mathrm{H}), 7.87(\mathrm{dd}, \mathrm{J}=7.2,1.2 \mathrm{~Hz}, 1 \mathrm{H}), 7.65-7.56(\mathrm{~m}, 3 \mathrm{H}) ;{ }^{13} \mathrm{C} \mathrm{NMR}(\mathrm{MeOD}) \delta 170.49,169.25$, $150.86,144.54,139.49,138.26,135.43,135.05,132.80,131.64,129.55,128.34,127.61$, 127.14, 126.39, 126.00; HRMS (ESI) calcd for $\mathrm{C}_{16} \mathrm{H}_{11} \mathrm{~N}_{3} \mathrm{O}_{3}$ [M-H] : 292.0728, found: 292.0746 (error $=6.16 \mathrm{ppm})$; decomposed at $200{ }^{\circ} \mathrm{C}$.

3-(Phenylamino)pyrazine-2-carboxylic acid (3). The title compound was obtained from the methyl ester in 69\% yield as a yellow solid. HPLC purity: 97.8\%; ${ }^{1} \mathrm{H}$ NMR (MeOD) $\delta 8.04$ $(\mathrm{d}, J=2.5 \mathrm{~Hz}, 1 \mathrm{H}), 7.80(\mathrm{~d}, J=2.6 \mathrm{~Hz}, 1 \mathrm{H}), 7.60(\mathrm{~d}, J=8.0 \mathrm{~Hz}, 2 \mathrm{H}), 7.21-7.16(\mathrm{~m}, 2 \mathrm{H})$, $6.88(\mathrm{td}, J=7.4,1.2 \mathrm{~Hz}, 1 \mathrm{H}) ;{ }^{13} \mathrm{C} \mathrm{NMR}(\mathrm{MeOD}) \delta 119.29,121.6,128.4,131.8,140.0,142.8$, 152.7; HRMS (ESI) calcd for $\mathrm{C}_{11} \mathrm{H}_{9} \mathrm{~N}_{3} \mathrm{O}_{2}[\mathrm{M}+2 \mathrm{Na}]^{+} 260.0406$, found: 260.0404 (error $=0.16$ ppm); decomposition temperature greater than $280{ }^{\circ} \mathrm{C}$.

3-(Benzaamido)pyrazine-2-carboxylic acid (5). The title compound was obtained from the methyl ester in $90 \%$ yield as a white powder. HPLC purity: $98.3 \% ;{ }^{1} \mathrm{H}$ NMR (DMSO- $\left.d_{6}\right) \delta$ $15.22(\mathrm{~s}, 1 \mathrm{H}), 8.37(\mathrm{~d}, \mathrm{~J}=2.5 \mathrm{~Hz}, 1 \mathrm{H}), 8.25(\mathrm{~d}, \mathrm{~J}=2.5 \mathrm{~Hz}, 1 \mathrm{H}), 8.05-7.98(\mathrm{~m}, 2 \mathrm{H}), 7.66-$ $7.59(\mathrm{~m}, 1 \mathrm{H}), 7.59-7.53(\mathrm{~m}, 2 \mathrm{H}) ;{ }^{13} \mathrm{C}$ NMR $\left(\mathrm{DMSO}_{-} d_{6}\right) \delta 166.59,163.37,149.61,142.58$, 138.12, 137.53, 135.04, 131.97, 128.78, 127.33; HRMS (ESI) calcd for $\mathrm{C}_{12} \mathrm{H}_{8} \mathrm{~N}_{3} \mathrm{O}_{3}[\mathrm{M}-\mathrm{H}]^{-}$: 242.0571, found: 242.0584 (error $=5.37 \mathrm{ppm}$ ); decomposed at $280{ }^{\circ} \mathrm{C}$.

3-Amino-2-iodopyrazine-2-carboxylic acid (6). The title compound was obtained from the methyl ester in $83 \%$ yield as a yellow solid. Spectra and other analytical data matched previously reported values for this compound (8). 
3-(Propionamido)pyrazine-2-carboxyclic acid (7). The title compound was obtained from the methyl ester in $38 \%$ yield as a white solid. HPLC purity: $99.2 \% ;{ }^{1} \mathrm{H}$ NMR (DMSO- $d_{6}$ ) $\delta$ $13.71(\mathrm{~s}, 1 \mathrm{H}), 8.26$ (d, J = 2.4 Hz, 1H), 8.16 (d, J = 2.5 Hz, 1H), 2.56 (q, J = $7.5 \mathrm{~Hz}, 2 \mathrm{H}), 1.09$ $(\mathrm{t}, \mathrm{J}=7.5 \mathrm{~Hz}, 3 \mathrm{H}) ;{ }^{13} \mathrm{C}$ NMR $\left(\mathrm{DMSO}-d_{6}\right) \delta 172.21,166.40,148.96,141.99,141.99,138.02$, 136.87, 136.87, 30.90, 9.19; HRMS (ESI) calcd for $\mathrm{C}_{8} \mathrm{H}_{8} \mathrm{~N}_{3} \mathrm{O}_{3}[\mathrm{M}-\mathrm{H}]$ : 194.0571 , found: 194.0579 (error $=4.12 \mathrm{ppm})$; decomposed at $260^{\circ} \mathrm{C}$.

3-(Ethylamino)pyrazine-2-carboxylic acid (8): The title compound was obtained from the methyl ester in $87 \%$ yield as a white solid. HPLC purity: $99.1 \% ;{ }^{1} \mathrm{H}$ NMR (MeOD) $\delta 7.87(\mathrm{~d}$, $J=2.6 \mathrm{~Hz}, 1 \mathrm{H}), 7.57(\mathrm{~d}, J=2.6 \mathrm{~Hz}, 1 \mathrm{H}), 3.33(\mathrm{q}, J=7.2 \mathrm{~Hz}, 2 \mathrm{H}), 1.16(\mathrm{t}, J=7.2 \mathrm{~Hz}, 3 \mathrm{H})$; ${ }^{13} \mathrm{C}$ NMR (MeOD) $\delta 171.0,155.1,143.1,133.8,129.1,34.8,13.5$; HRMS (ESI) calcd for $\mathrm{C}_{7} \mathrm{H}_{9} \mathrm{~N}_{3} \mathrm{O}_{2}[\mathrm{M}+2 \mathrm{Na}]^{+}: 212.0456$, found: 212.0471 (error $=8.02 \mathrm{ppm}$ ); Mp: $133-136{ }^{\circ} \mathrm{C}$.

3-(Isobutylamino)pyrazine-2-carboxylic acid (9). The title compound was obtained from the methyl ester in 97\% yield as an off-white solid. HPLC purity: 99.7\%; ${ }^{1} \mathrm{H}$ NMR (DMSO$\left.d_{6}\right) \delta 10.06(\mathrm{t}, \mathrm{J}=5.7 \mathrm{~Hz}, 1 \mathrm{H}), 7.88(\mathrm{~d}, \mathrm{~J}=2.6 \mathrm{~Hz}, 1 \mathrm{H}), 7.53(\mathrm{~d}, \mathrm{~J}=2.6 \mathrm{~Hz}, 1 \mathrm{H}), 3.19-3.11$ $(\mathrm{m}, 2 \mathrm{H}), 1.87-1.76(\mathrm{~m}, 1 \mathrm{H}), 0.91(\mathrm{~d}, \mathrm{~J}=6.7 \mathrm{~Hz}, 6 \mathrm{H}) ;{ }^{13} \mathrm{C}$ NMR (DMSO- $\left.d_{6}\right) \delta 168.06$, 155.46, 142.21, 135.59, 128.80, 47.40, 27.75, 20.28; HRMS (ESI) calcd for $\mathrm{C}_{9} \mathrm{H}_{12} \mathrm{~N}_{3} \mathrm{O}_{2}$ [M$\mathrm{H}]^{-}: 194.0935$, found: 194.0944 (error $=4.64 \mathrm{ppm}$ ); decomposition temperature greater than $280{ }^{\circ} \mathrm{C}$.

3-(Cyclobutylamino)pyrazine-2-carboxylic acid (10): The title compound was obtained from the methyl ester in $86 \%$ yield as a white solid. HPLC purity: 95.8\%; ${ }^{1} \mathrm{H}$ NMR (MeOD) $\delta 7.85(\mathrm{~d}, J=2.6 \mathrm{~Hz}, 1 \mathrm{H}), 7.58(\mathrm{~d}, J=2.6 \mathrm{~Hz}, 1 \mathrm{H}), 4.34(\mathrm{p}, J=8.0 \mathrm{~Hz}, 1 \mathrm{H}), 2.32$ (dtt, $J=$ 12.8, 8.0, $2.6 \mathrm{~Hz}, 2 \mathrm{H}), 1.94-1.84(\mathrm{~m}, 1 \mathrm{H}), 1.78-1.64(\mathrm{~m}, 2 \mathrm{H}) ;{ }^{13} \mathrm{C} \mathrm{NMR}(\mathrm{MeOD}) \delta 170.9$, 154.1, 143.2, 133.5, 129.4, 45.6, 30.8, 14.8; HRMS (ESI) calcd for $\mathrm{C}_{9} \mathrm{H}_{11} \mathrm{~N}_{3} \mathrm{O}_{2} \mathrm{Na}_{2}[\mathrm{M}+2 \mathrm{Na}]^{+}$: 238.0563, found: 238.0566 (error $=1.46 \mathrm{ppm}$ ); decomposition temperature greater than 280 ${ }^{\circ} \mathrm{C}$.

3-(4-Fluorophenyl)pyrazine-2-carboxylic acid (11). The title compound was obtained from the methyl ester in 90\% yield as a tan solid. HPLC purity: 98.8\%; ${ }^{1} \mathrm{H}$ NMR (DMSO- $\left.d_{6}\right) \delta$ $8.45(\mathrm{~d}, \mathrm{~J}=2.5 \mathrm{~Hz}, 1 \mathrm{H}), 8.34(\mathrm{~d}, \mathrm{~J}=2.5 \mathrm{~Hz}, 1 \mathrm{H}), 8.07-7.98(\mathrm{~m}, 2 \mathrm{H}), 7.27-7.18(\mathrm{~m}, 2 \mathrm{H}$; ${ }^{13} \mathrm{C}$ NMR (DMSO- $\left.d_{6}\right) \delta 169.95,163.43,161.48,155.74,145.93,141.47,140.75,134.90$, 130.56, 130.50, 114.83, 114.66; HRMS (ESI) calcd for $\mathrm{C}_{11} \mathrm{H}_{6} \mathrm{FN}_{2} \mathrm{O}_{2}$ [M-H]: 217.0419, found: 217.0431 (error $=5.53 \mathrm{ppm})$; decomposition temperature greater than $280^{\circ} \mathrm{C}$.

3-(2-Naphthamido)pyrazine-2-carboxylic acid (12). The title compound was obtained from the methyl ester in 44\% yield as an off-white solid. HPLC purity: 96.7\%; ${ }^{1} \mathrm{H}$ NMR (MeOD) $\delta$ $8.68(\mathrm{~s}, 1 \mathrm{H}), 8.45(\mathrm{~d}, \mathrm{~J}=2.5 \mathrm{~Hz}, 1 \mathrm{H}), 8.39$ (d, J = 2.5 Hz, 1H), $8.16(\mathrm{dd}, \mathrm{J}=8.6,1.9 \mathrm{~Hz}, 1 \mathrm{H})$, $8.12(\mathrm{~d}, \mathrm{~J}=7.9 \mathrm{~Hz}, 1 \mathrm{H}), 8.03(\mathrm{~d}, \mathrm{~J}=8.6 \mathrm{~Hz}, 1 \mathrm{H}), 7.97(\mathrm{~d}, \mathrm{~J}=7.9 \mathrm{~Hz}, 1 \mathrm{H}), 7.62(\mathrm{dqd}, \mathrm{J}=8.2$, 6.9, $1.4 \mathrm{~Hz}, 2 \mathrm{H}$ ); ${ }^{13} \mathrm{C}$ NMR (MeOD) $\delta 170.70,166.76,151.02,144.53,139.24,136.73$, 134.20, 132.96, 130.50, 129.78, 129.62, 129.36, 128.78, 128.01, 124.92; HRMS (ESI) calcd for $\mathrm{C}_{16} \mathrm{H}_{10} \mathrm{~N}_{3} \mathrm{O}_{3}[\mathrm{M}-\mathrm{H}]$ : 292.0728 , found: 292.0742 (error $=4.79 \mathrm{ppm}$ ); decomposed at 230 ${ }^{\circ} \mathrm{C}$. 
3-(Diethylamino)pyrazine-2-carboxylic acid (13). The title compound was obtained from the methyl ester in quantitative yield as a yellow solid. HPLC purity: $95.8 \%$; ${ }^{1} \mathrm{H}$ NMR $(\mathrm{MeOD}) \delta 7.95(\mathrm{~d}, \mathrm{~J}=2.6 \mathrm{~Hz}, 1 \mathrm{H}), 7.58(\mathrm{~d}, \mathrm{~J}=2.6 \mathrm{~Hz}, 1 \mathrm{H}), 3.58(\mathrm{q}, \mathrm{J}=7.0 \mathrm{~Hz}, 4 \mathrm{H}), 1.20$ (t, $\mathrm{J}=7.0 \mathrm{~Hz}, 6 \mathrm{H}), ;{ }^{13} \mathrm{C} \mathrm{NMR}(\mathrm{MeOD}) \delta 175.42,152.56,142.88,141.32,130.13,44.26,13.22$; HRMS (ESI) calcd for $\mathrm{C}_{9} \mathrm{H}_{12} \mathrm{~N}_{3} \mathrm{O}_{2}[\mathrm{M}-\mathrm{H}]^{-}: 194.0935$, found: 194.0945 (error $=5.15 \mathrm{ppm}$ ); decomposed at $240{ }^{\circ} \mathrm{C}$.

3-Phenylpyrazine-2-carboxylic acid (14). The title compound was obtained from the methyl ester in $66 \%$ yield as a white solid. Spectra and other analytical data matched previously reported values for this compound (9).

3-(4-(Methylthio)phenyl)pyrazine-2-carboxylic acid (15). The title compound was obtained from the methyl ester in $56 \%$ yield as an off-white solid. HPLC purity: $90.7 \% ;{ }^{1} \mathrm{H}$ NMR (MeOD) $\delta 8.56(\mathrm{~d}, \mathrm{~J}=2.6 \mathrm{~Hz}, 1 \mathrm{H}), 8.41(\mathrm{~d}, \mathrm{~J}=2.6 \mathrm{~Hz}, 1 \mathrm{H}), 7.87-7.80(\mathrm{~m}, 2 \mathrm{H}), 7.35$ - 7.30 (m, 2H), 2.51 (s, 3H); ${ }^{13} \mathrm{C}$ NMR (MeOD) $\delta 154.07,150.64,146.77,143.87,142.29$, 142.14, 135.29, 130.90, 130.13, 126.75, 124.79, 15.19; HRMS (ESI) calcd for $\mathrm{C}_{12} \mathrm{H}_{10} \mathrm{~N}_{2} \mathrm{O}_{2} \mathrm{~S}$ [M-H]: 245.0390 , found: 245.0402 (error $=4.90 \mathrm{ppm}$ ); decomposed at $270{ }^{\circ} \mathrm{C}$.

3-(o-Tolyl)pyrazine-2-carboxylic acid (16). The title compound was obtained from the methyl ester in $92 \%$ yield as a tan solid. HPLC purity: 98.2\%; ${ }^{1} \mathrm{H}$ NMR (MeOD) $\delta 8.56(\mathrm{~d}, \mathrm{~J}$ $=2.6 \mathrm{~Hz}, 1 \mathrm{H}), 8.50(\mathrm{~d}, \mathrm{~J}=2.6 \mathrm{~Hz}, 1 \mathrm{H}), 7.39-7.34(\mathrm{~m}, 1 \mathrm{H}), 7.33-7.24(\mathrm{~m}, 2 \mathrm{H}), 7.21(\mathrm{td}, \mathrm{J}$ $=7.3,1.7 \mathrm{~Hz}, 1 \mathrm{H}), 2.23(\mathrm{~s}, 3 \mathrm{H}) ;{ }^{13} \mathrm{C} \mathrm{NMR}(\mathrm{MeOD}) \delta 173.55,155.18,153.26,143.34$, 142.98, 138.98, 137.84, 131.27, 130.34, 129.74, 126.35, 20.12; HRMS (ESI) calcd for $\mathrm{C}_{12} \mathrm{H}_{9} \mathrm{~N}_{2} \mathrm{O}_{2}$ [M-H] : 213.0670, found: 213.0684 (error $=6.57$ ppm); decomposition temperature greater than $280{ }^{\circ} \mathrm{C}$.

3-(Benzylamino)pyrazine-2-carboxylic acid (17): The title compound was obtained from Methyl 6-chloropyrazinoate in $74 \%$ yield as a white solid. HPLC purity: $98.8 \% ;{ }^{1} \mathrm{H}$ NMR (MeOD) $\delta 7.89$ (d, $J=2.6 \mathrm{~Hz}, 1 \mathrm{H}), 7.62(\mathrm{~d}, J=2.6 \mathrm{~Hz}, 1 \mathrm{H}), 7.26(\mathrm{~d}, J=7.1 \mathrm{~Hz}, 2 \mathrm{H}), 7.22-$ $7.18(\mathrm{~m}, 2 \mathrm{H}), 7.15-7.10(\mathrm{~m}, 1 \mathrm{H}), 4.54(\mathrm{~s}, 2 \mathrm{H}) ;{ }^{13} \mathrm{C}$ NMR (MeOD) $\delta 171.0,155.0,143.2,139$. 5, 129.7, 128.1, 127.0, 126.5, 43.9; HRMS (ESI) calcd for $\mathrm{C}_{12} \mathrm{H}_{11} \mathrm{~N}_{3} \mathrm{O}_{2}[\mathrm{M}+2 \mathrm{Na}]^{+}$274.0563, found: 274.0564 (error $=0.08 \mathrm{ppm}$ ); decomposition temperature greater than $280{ }^{\circ} \mathrm{C}$.

3-(Cyclopentylamino)pyrazine-2-carboxylic acid (18): The title compound was obtained from the ester in 78\% yield as an off- white solid. HPLC purity: 99.3\%; ${ }^{1} \mathrm{H}$ NMR (MeOD) $\delta$ $8.16(\mathrm{~d}, J=2.2 \mathrm{~Hz}, 1 \mathrm{H}), 7.67$ (d, $J=2.2 \mathrm{~Hz}, 1 \mathrm{H}), 4.27$ (q, $J=6.5 \mathrm{~Hz}, 1 \mathrm{H}), 1.97$ (dq, $J=12.9$, $6.8 \mathrm{~Hz}, 2 \mathrm{H}), 1.71-1.62(\mathrm{~m}, 2 \mathrm{H}), 1.58(\mathrm{qd}, J=9.5,7.7,5.2 \mathrm{~Hz}, 2 \mathrm{H}), 1.47-1.36(\mathrm{~m}, 2 \mathrm{H}) ;{ }^{13} \mathrm{C}$ NMR (MeOD) $\delta 168.1,155.0,147.6,129.9,123.9$, 52.0, 32.7, 23.3; HRMS (ESI) calcd for $\mathrm{C}_{10} \mathrm{H}_{13} \mathrm{~N}_{3} \mathrm{O}_{2}[\mathrm{M}+2 \mathrm{Na}]^{+}:$252.0719, found: 252.0701 (error $=6.85 \mathrm{ppm}$ ); Mp: $99-101{ }^{\circ} \mathrm{C}$.

3-(Quinoline-2-carboxamido)pyrazine-2-carboxylic acid (19). The title compound was obtained from the methyl ester in $85 \%$ yield as a white solid. HPLC purity: $99.2 \%$; ${ }^{1} \mathrm{H}$ NMR $(\mathrm{MeOD}) \delta 8.51(\mathrm{~d}, \mathrm{~J}=8.5 \mathrm{~Hz}, 1 \mathrm{H}), 8.49-8.43(\mathrm{~m}, 2 \mathrm{H}), 8.40(\mathrm{~d}, \mathrm{~J}=2.5 \mathrm{~Hz}, 1 \mathrm{H}), 8.35(\mathrm{~d}, \mathrm{~J}=$ $8.4 \mathrm{~Hz}, 1 \mathrm{H}), 8.01(\mathrm{dd}, \mathrm{J}=8.3,1.4 \mathrm{~Hz}, 1 \mathrm{H}), 7.86$ (ddd, J = 8.4, 6.8, $1.4 \mathrm{~Hz}, 1 \mathrm{H}), 7.71$ (ddd, J = 8.2, 6.9, $1.2 \mathrm{~Hz}, 1 \mathrm{H}) ;{ }^{13} \mathrm{C} \mathrm{NMR}(\mathrm{MeOD}) \delta 170.68,164.99,150.98,150.15,148.16,144.21$, $139.37,139.00,131.67,131.46,130.96,129.69$, 128.78, 119.72; HRMS (ESI) calcd for 
$\mathrm{C}_{15} \mathrm{H}_{9} \mathrm{~N}_{4} \mathrm{O}_{3}[\mathrm{M}-\mathrm{H}]^{-}:$293.0680, found: 293.0693 (error $=4.44$ ppm); decomposition temperature beyond $280{ }^{\circ} \mathrm{C}$.

3-(Benzofuran-2-carboxamido)pyrazine-2-carboxylic acid (20). The title compound was obtained from the methyl ester in 52\% yield as a tan solid. HPLC purity: $89.9 \% ;{ }^{1} \mathrm{H}$ NMR $\left(\right.$ DMSO- $\left.d_{6}\right) \delta 15.50(\mathrm{~s}, 1 \mathrm{H}), 8.41(\mathrm{~d}, \mathrm{~J}=2.4 \mathrm{~Hz}, 1 \mathrm{H}), 8.28(\mathrm{~d}, \mathrm{~J}=2.5 \mathrm{~Hz}, 1 \mathrm{H}), 7.84(\mathrm{~d}, \mathrm{~J}=7.8$ $\mathrm{Hz}, 1 \mathrm{H}), 7.74-7.65(\mathrm{~m}, 2 \mathrm{H}), 7.56-7.49(\mathrm{~m}, 1 \mathrm{H}), 7.38(\mathrm{t}, \mathrm{J}=7.5 \mathrm{~Hz}, 1 \mathrm{H}) ;{ }^{13} \mathrm{C} \mathrm{NMR}$ $\left(\right.$ DMSO$\left._{-} d_{6}\right) \delta 167.00,155.64,155.01,149.85,149.66,143.28,138.44,127.91,127.72$, 127.62, 124.45, 124.29, 123.52, 123.44, 112.54, 112.38, 111.18, 111.00; HRMS (ESI) calcd for $\mathrm{C}_{14} \mathrm{H}_{8} \mathrm{~N}_{3} \mathrm{O}_{4}[\mathrm{M}-\mathrm{H}]^{-}: 282.0520$, found: 282.0532 (error $=4.25 \mathrm{ppm}$ ) ; decomposed at 240 ${ }^{\circ} \mathrm{C}$.

\section{Part III: References}

(1) Q. Sun, Li, X., Perez, L. M., Shi, W., Zhang, Y., Sacchettini, J. C. (2020) The molecular basis of pyrazinamide activity on Mycobacterium tuberculosis PanD. Nat. Comm. 11:339, doi.org/10.1038/s41467-019-14238-3.

(2) B. I. Lee, Suh, S. W. (2004) Crystal Structure of the Schiff Base Intermediate Prior to Decarboxylation in the Catalytic Cycle of Aspartate $\alpha$-Decarboxylase. J. Mol. Biol. 340, 1-7.

(3) Meng, W., Zhang, H., Guo, T., Pandey, C., Zhu, Y., Kon, O. L., and Sze, S. K. (2008) One-Step Procedure for Peptide Extraction from In-Gel Digestion Sample for Mass Spectrometric Analysis. Anal. Chem. 80, 9797-9805.

(4) P. Norcott, P. J. Rayner, G. G. R. Green, S. B. Duckett. (2017) Achieving High ${ }^{1}$ H Nuclear Hyperpolarization Levels with Long Lifetimes in a Range of Tuberculosis Drug Scaffolds. Chem. Eur. J. 23, 16990-16998.

(5) H. Duan, M. Ning, X. Chen, Q. Zou, L. Zhang, Y. Feng, L. Zhang, Y. Leng, and J. Shen. (2012) Design, Synthesis, and Antidiabetic Activity of 4-Phenoxynicotinamide and 4Phenoxypyrimidine-5-carboxamide Derivatives as Potent and Orally Efficacious TGR5 Agonists. J. Med. Chem. 55 (23), 10475-10489.

(6) K. Komeyama, Y. Nagao, M. Abe, K Takaki. (2014) Scope and Limitation for $\mathrm{FeSO}_{4}{ }^{-}$ Mediated Direct Arylation of Heteroarenes with Arylboronic Acids and Its Synthetic Applications. Bull. Chem. Soc. Japan. 87 (2), 301 - 313.

(7) X. Dai, H. Liu, A. Palani, S. He, R Nargund, D. Xiao, N. Zorn, Q. Dang, C.C. McComas, X. Peng, P. Li, R. Soll. (2014) Substituted benzofuran compounds and methods of use thereof for the treatment of viral diseases. U.S. Patent WO2014/209727, A1.

(8) W.D. Arnold, A. Gosberg, Z. Li, R.W. Steensma, M.E. Wilson. (2006) Fused ring heterocycle kinase modulators. U.S. Patent WO2006/15124, A2.

(9) G.B. Barlin. (1984) Heterocyclic amplifiers of phleomycin. II. Thiazol-4'-ylpyrazines with phenyl substituents and strongly basic side chains. Aus. J. Chem. 37(5), 1049-1056. 
Part IV: NMR Spectra<smiles>COC(=O)c1nccnc1Cl</smiles>

Methyl 3-chloropyrazine-2-carboxylate

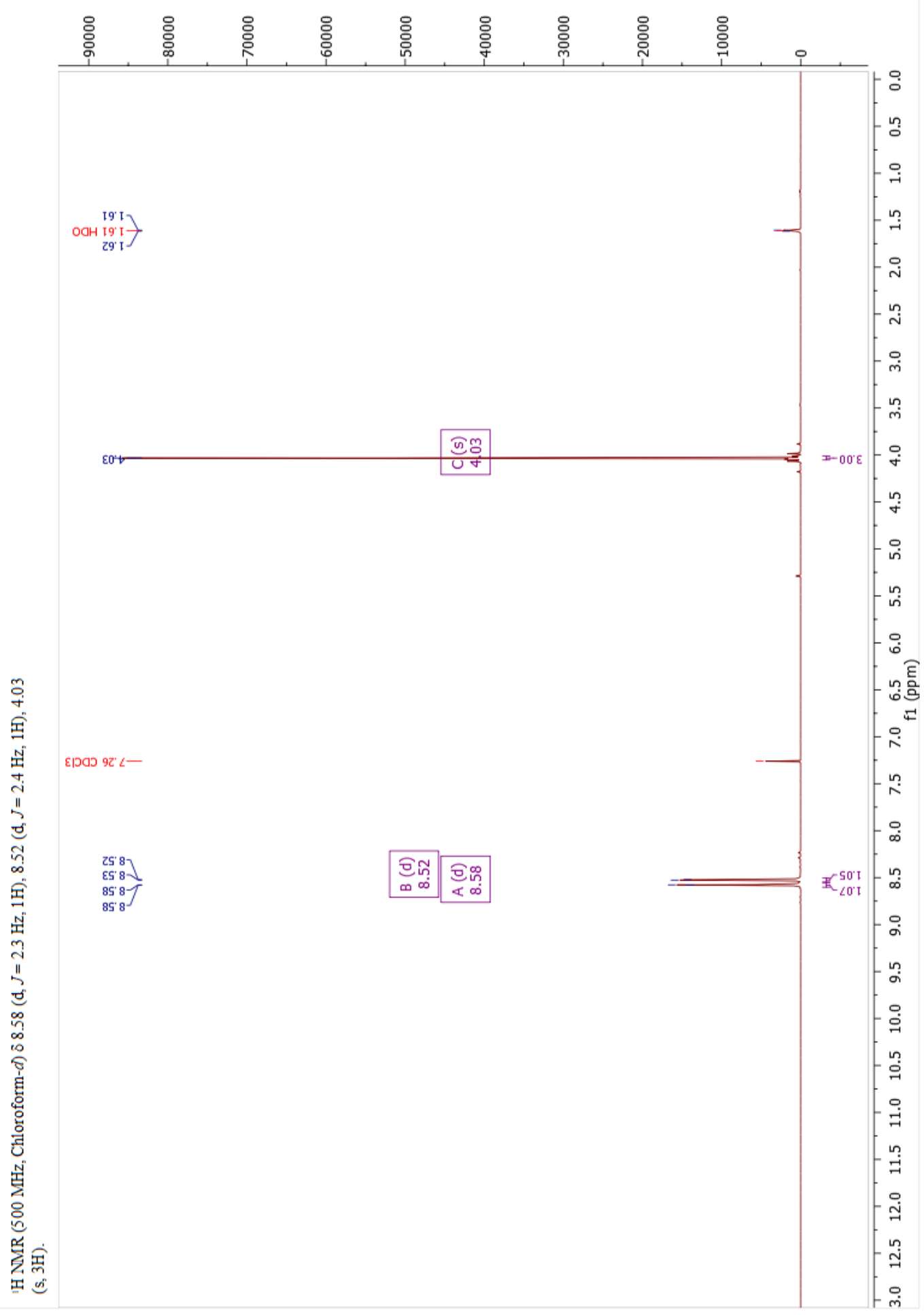




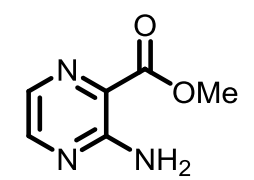

Methyl 3-aminopyrazine-2-carboxylate

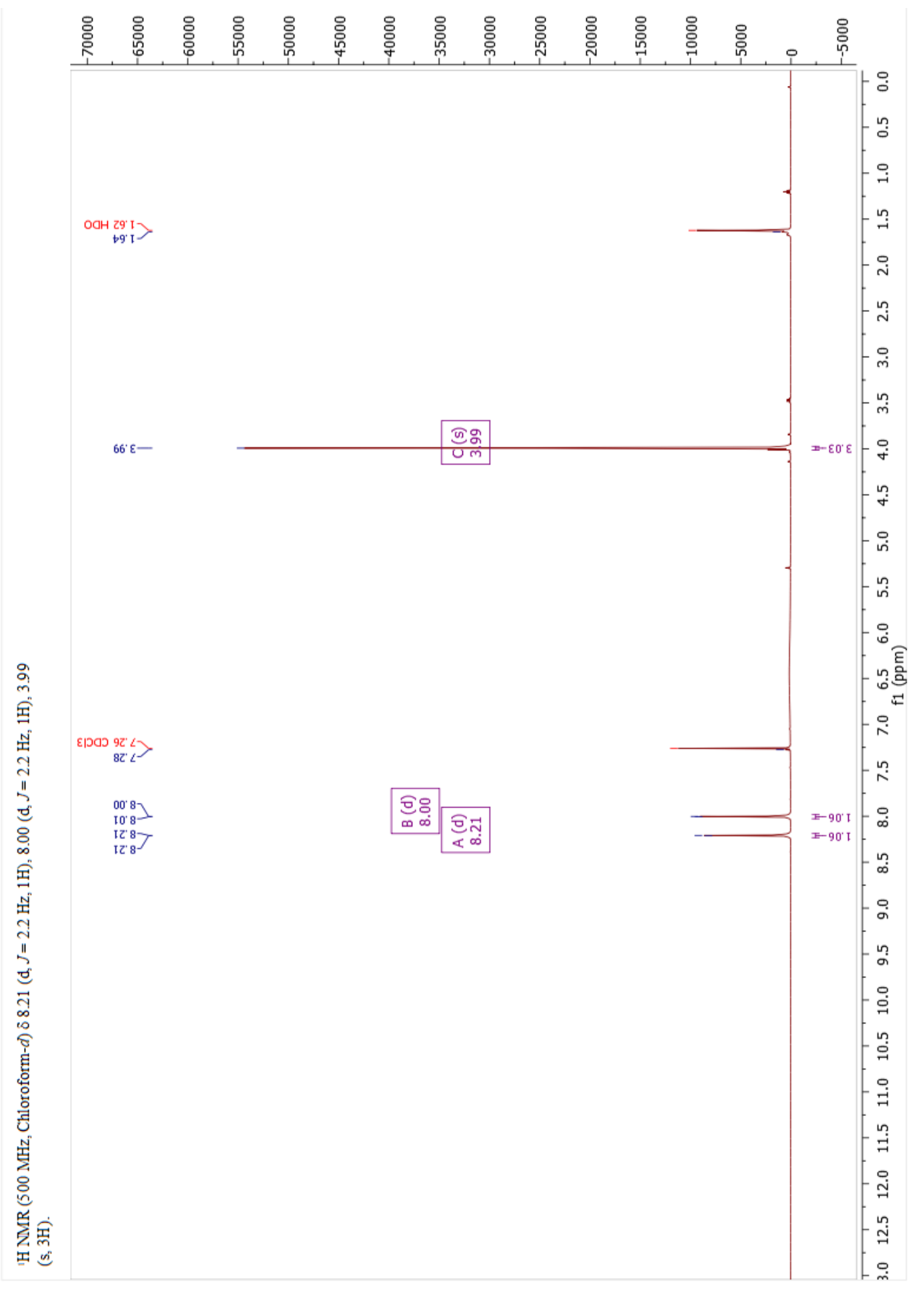


<smiles>Nc1ncc(Br)nc1C(=O)O</smiles>

3-Amino-6-bromopyrazine-2-carboxylic acid (1)

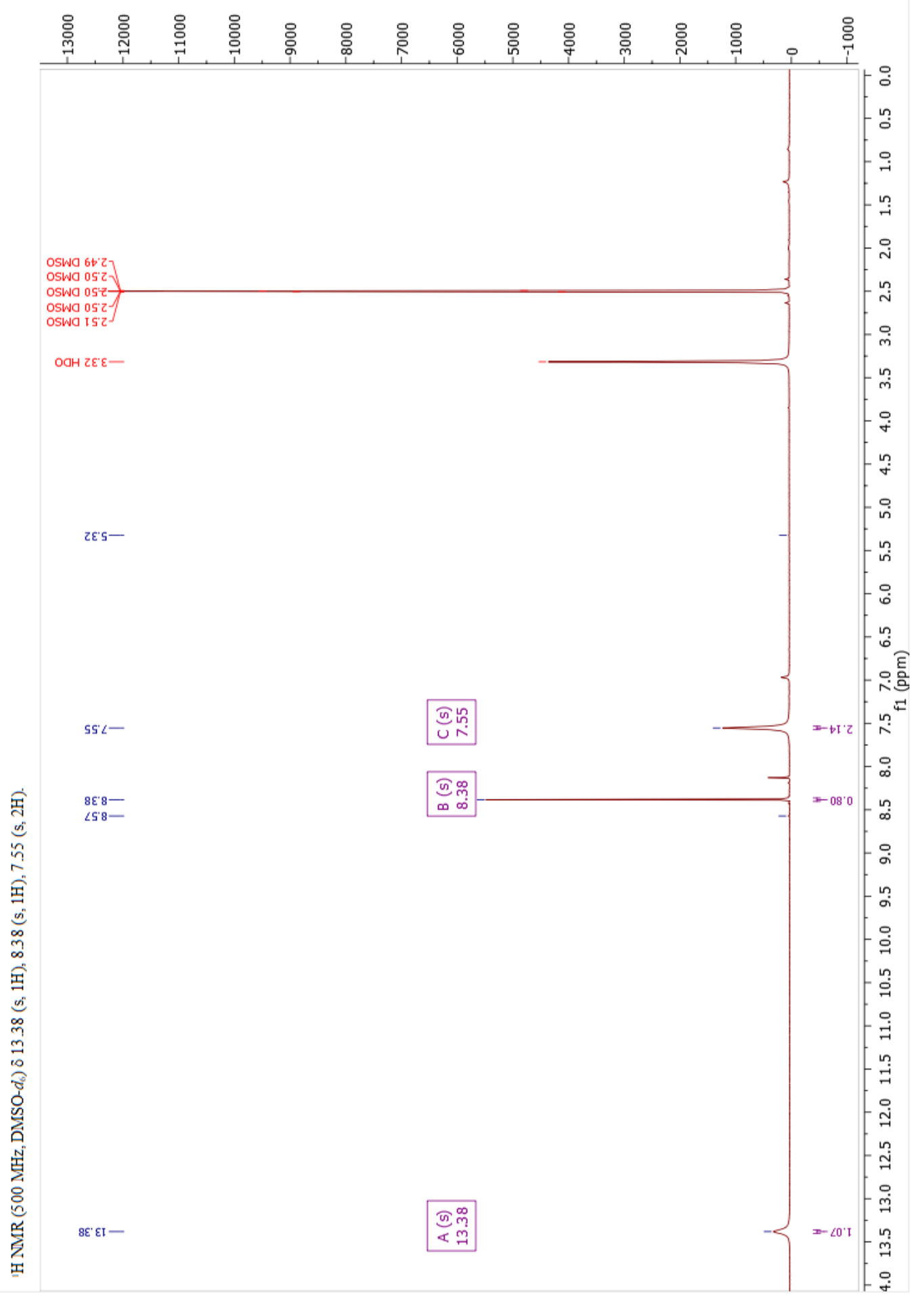




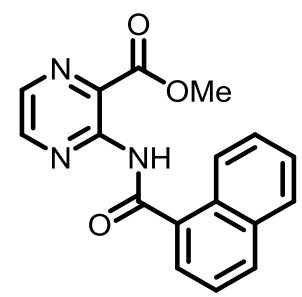

Methyl 3-(1-naphthamido)pyrazine-2-carboxylate (2-OMe)

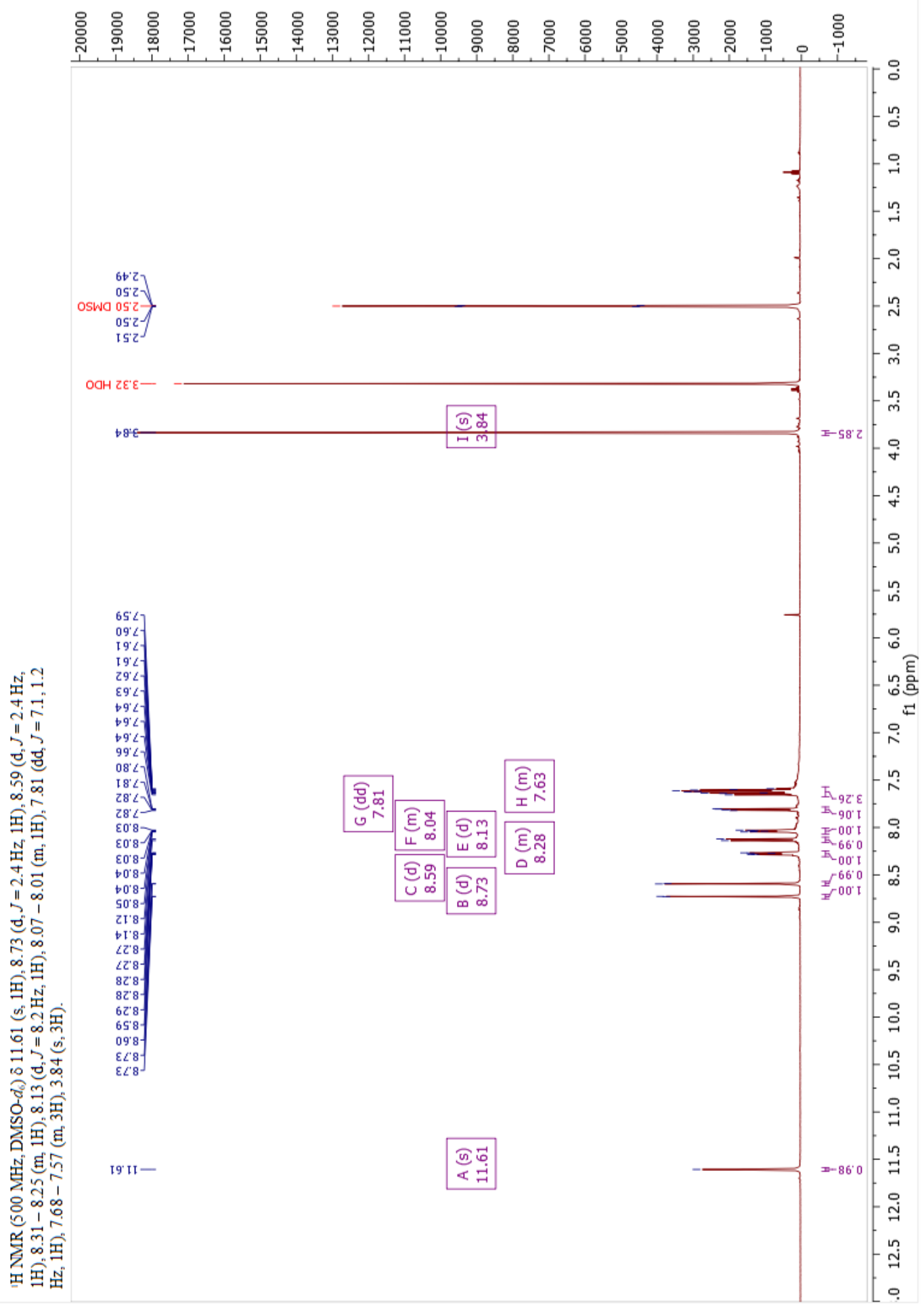




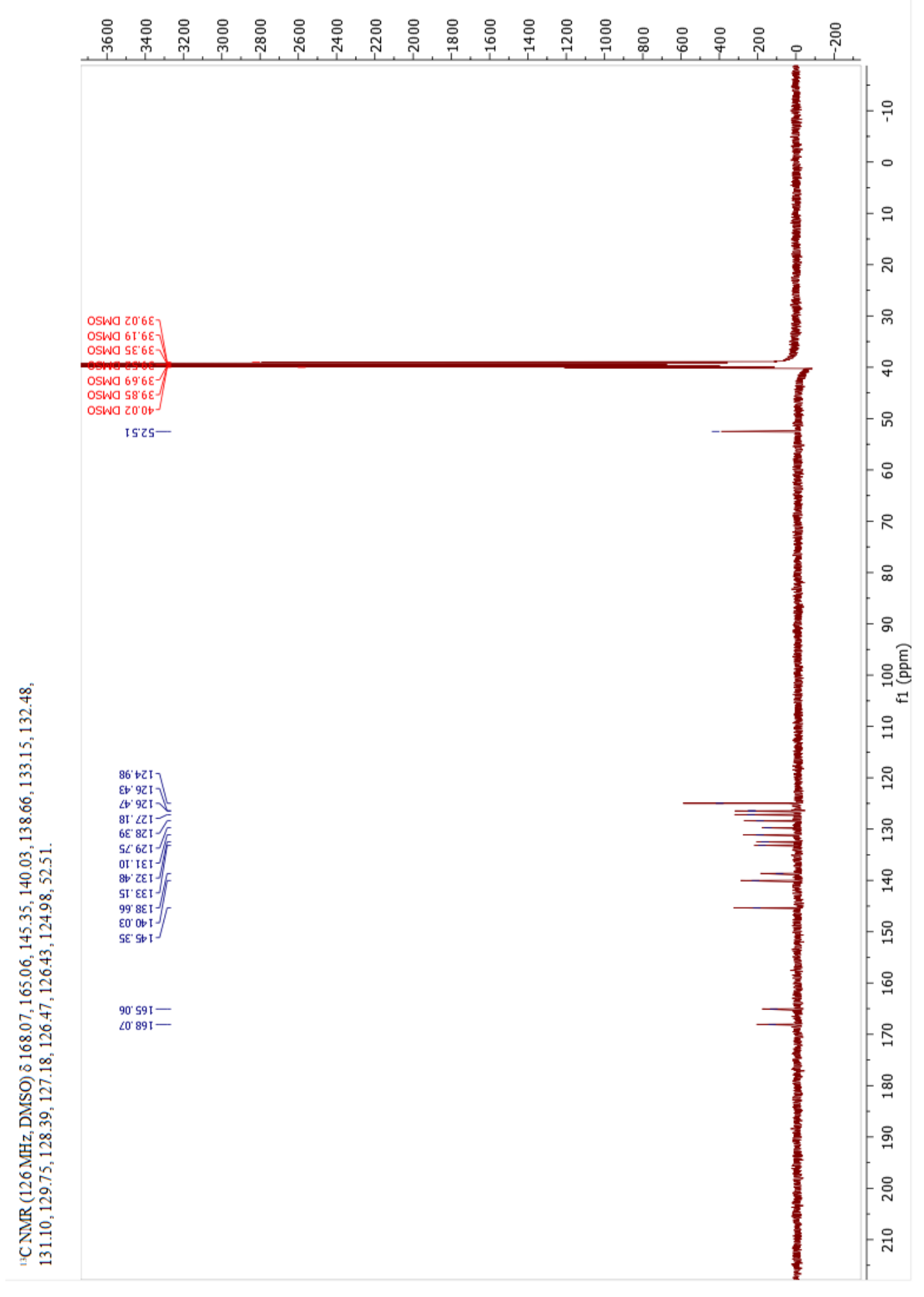


<smiles>O=C(O)c1nccnc1NC(=O)c1cccc2ccccc12</smiles>

3-(1-Naphthamido)pyrazine-2-carboxylic acid (2)

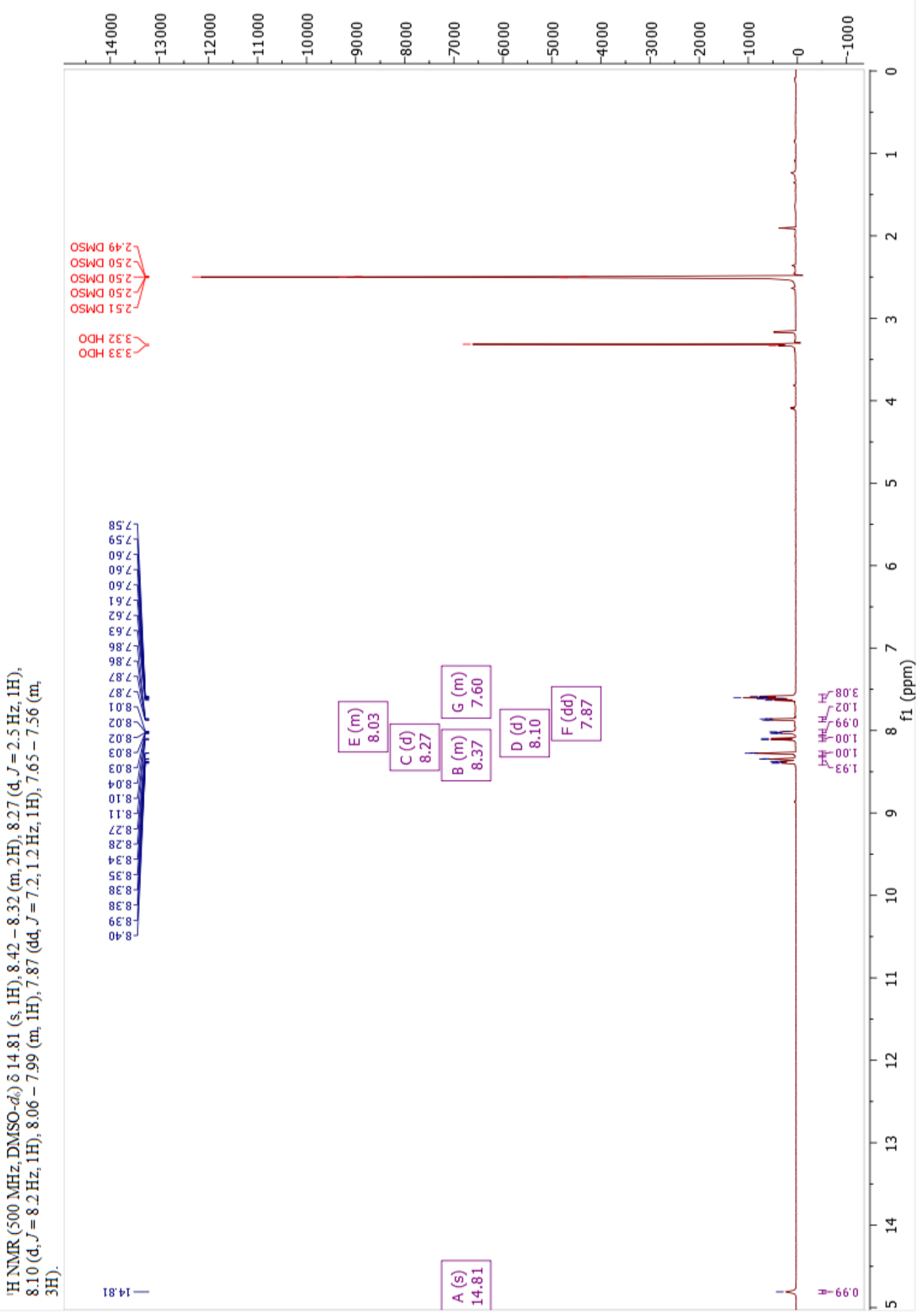




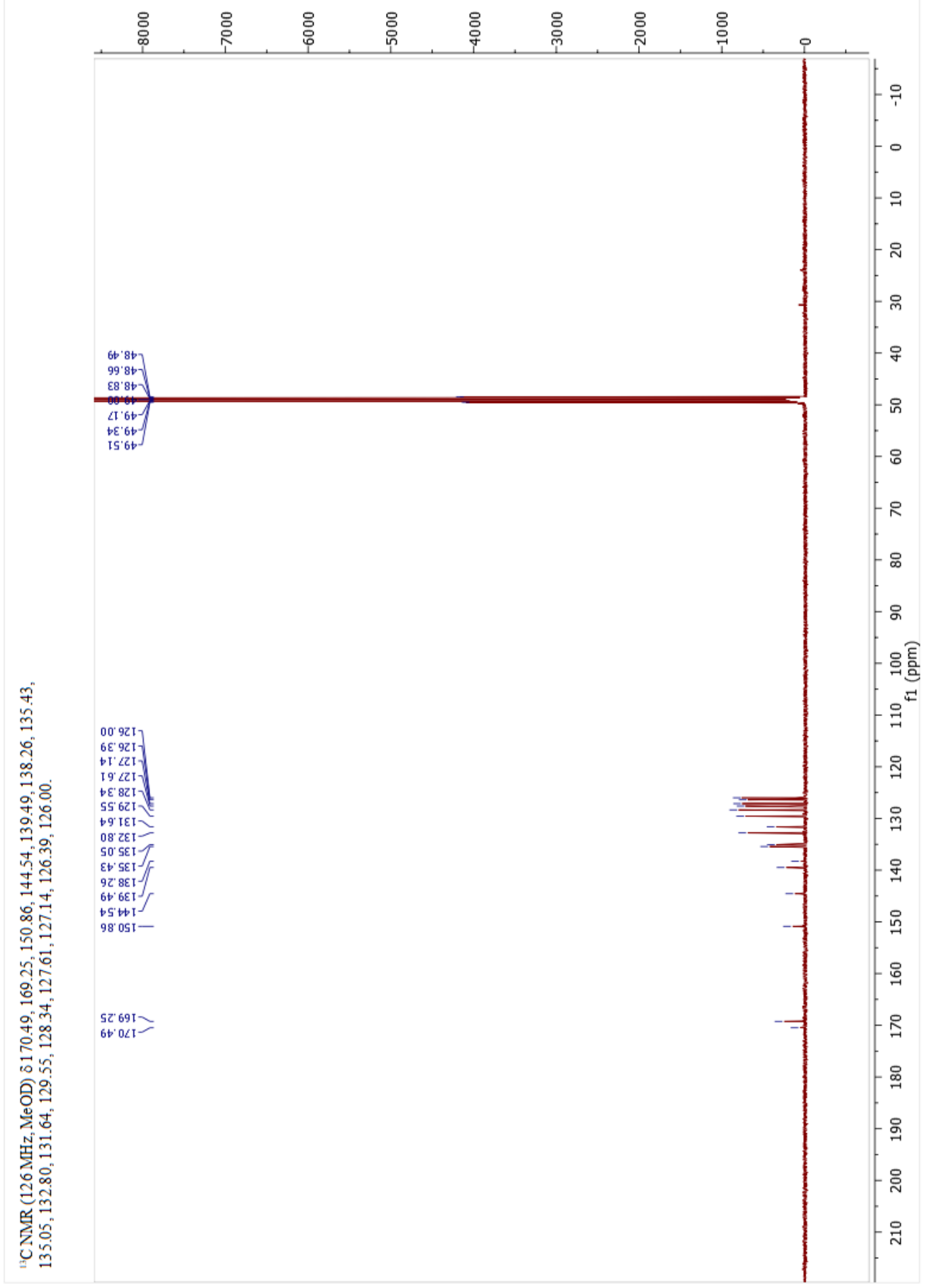


<smiles>COC(=O)c1nccnc1Nc1ccccc1</smiles>

3-(Phenylamino)pyrazine-2-carboxylic acid (3)

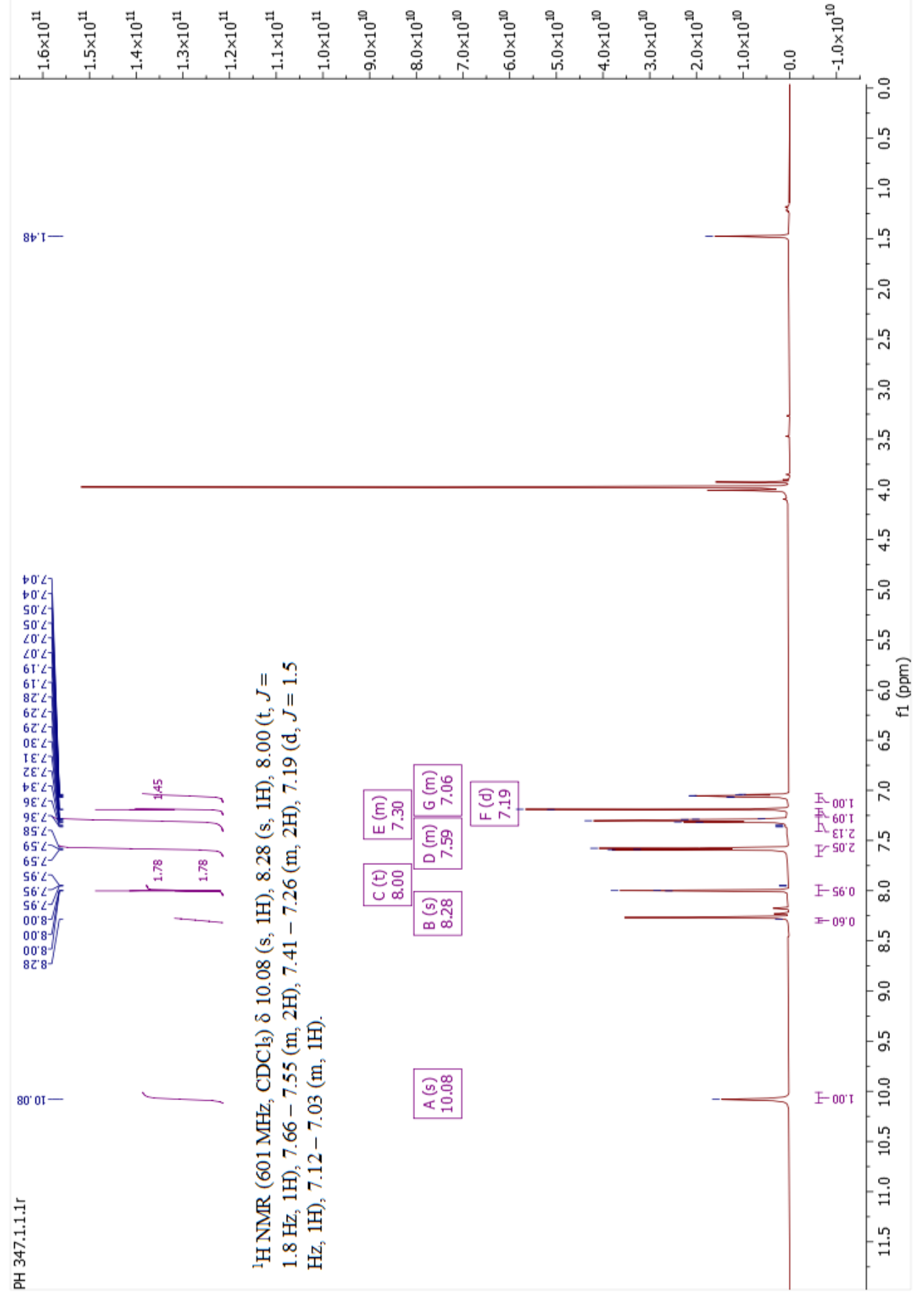




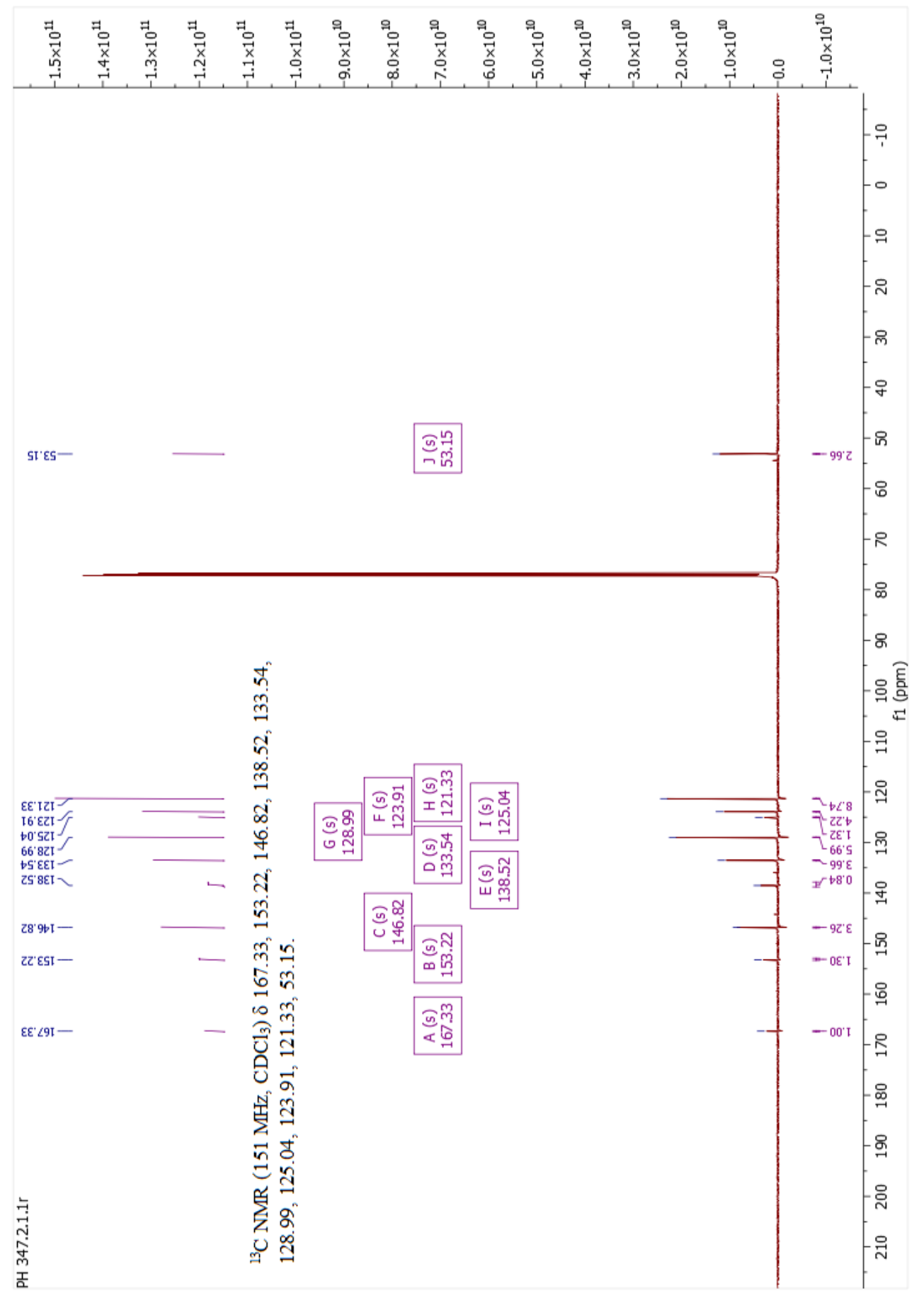


<smiles>O=C(O)c1nccnc1Nc1ccccc1</smiles>

3-(Phenylamino)pyrazine-2-carboxylic acid (3)

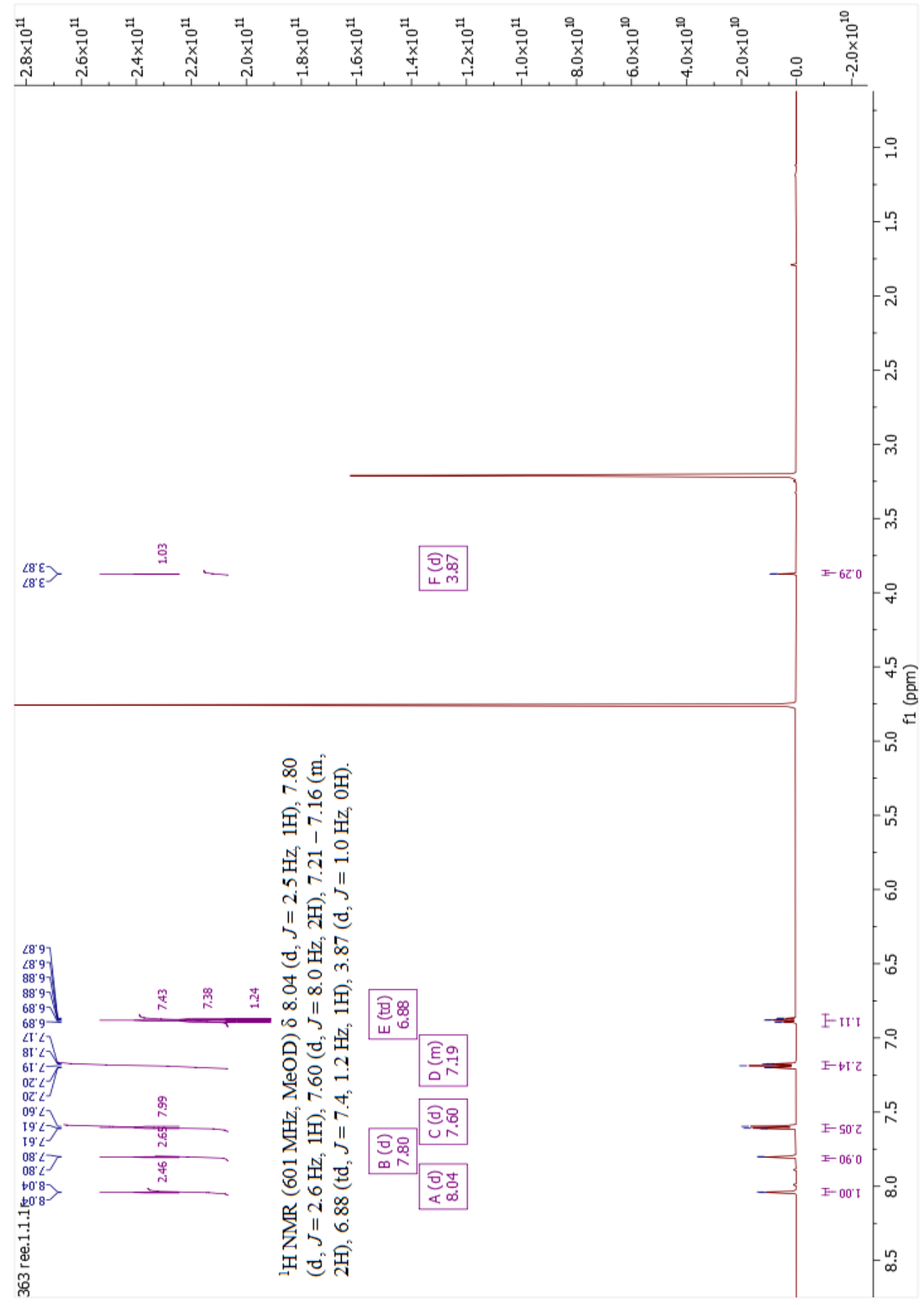




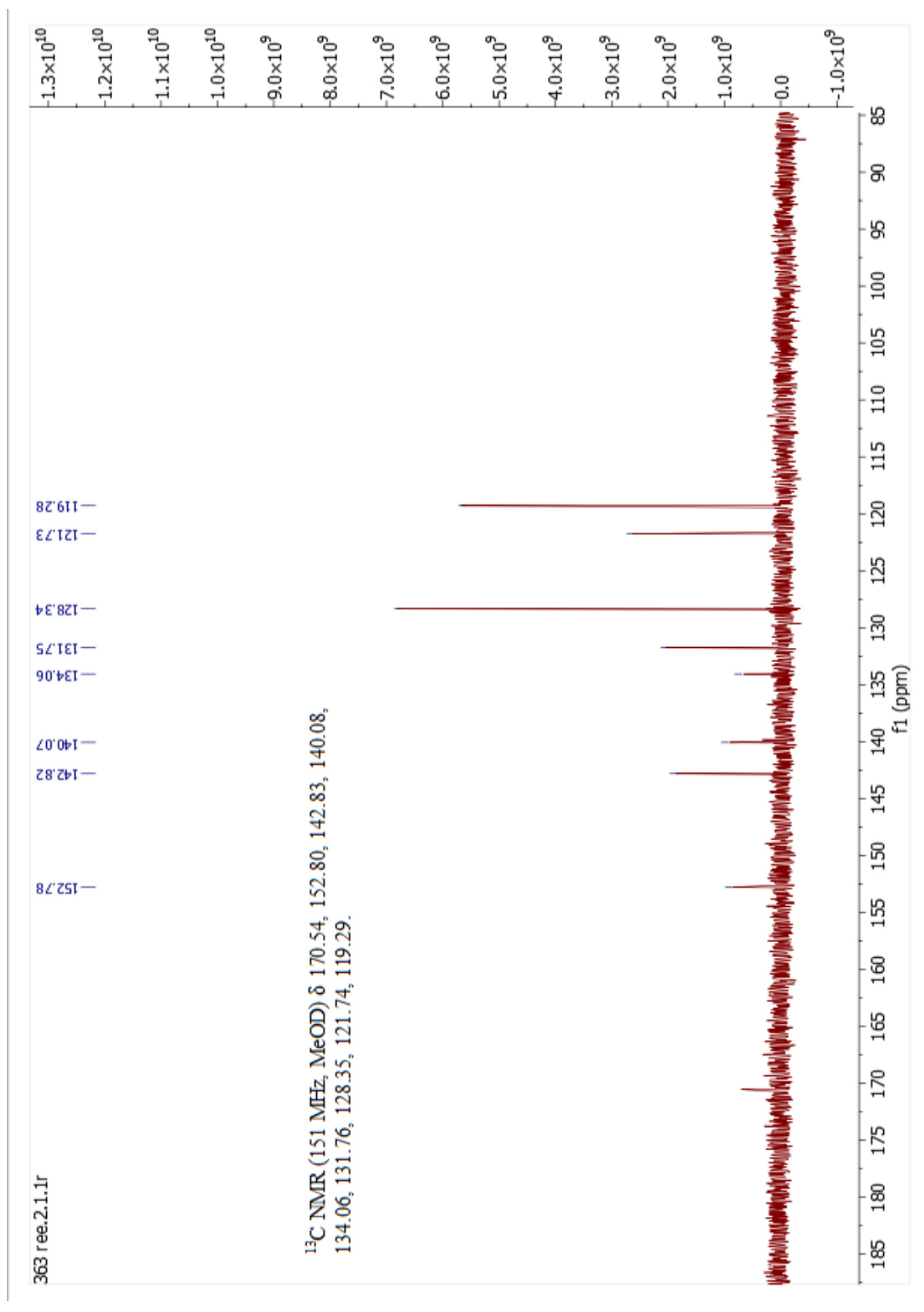




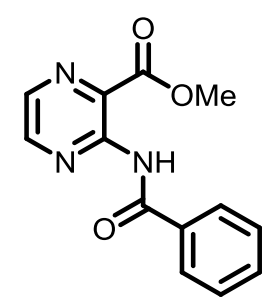

Methyl 3-benzamidopyrazine-2-carboxylic acid (5-OMe)

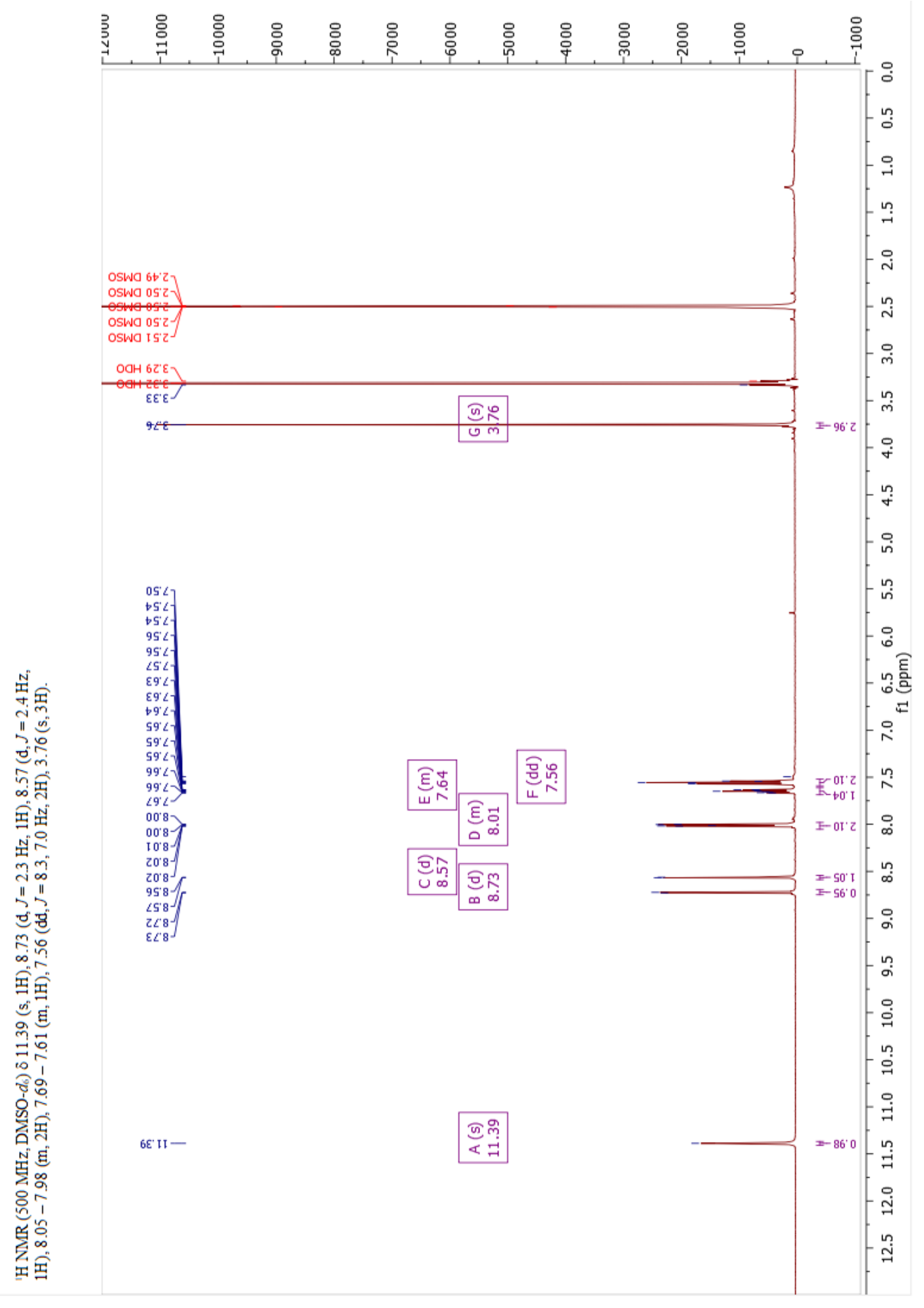




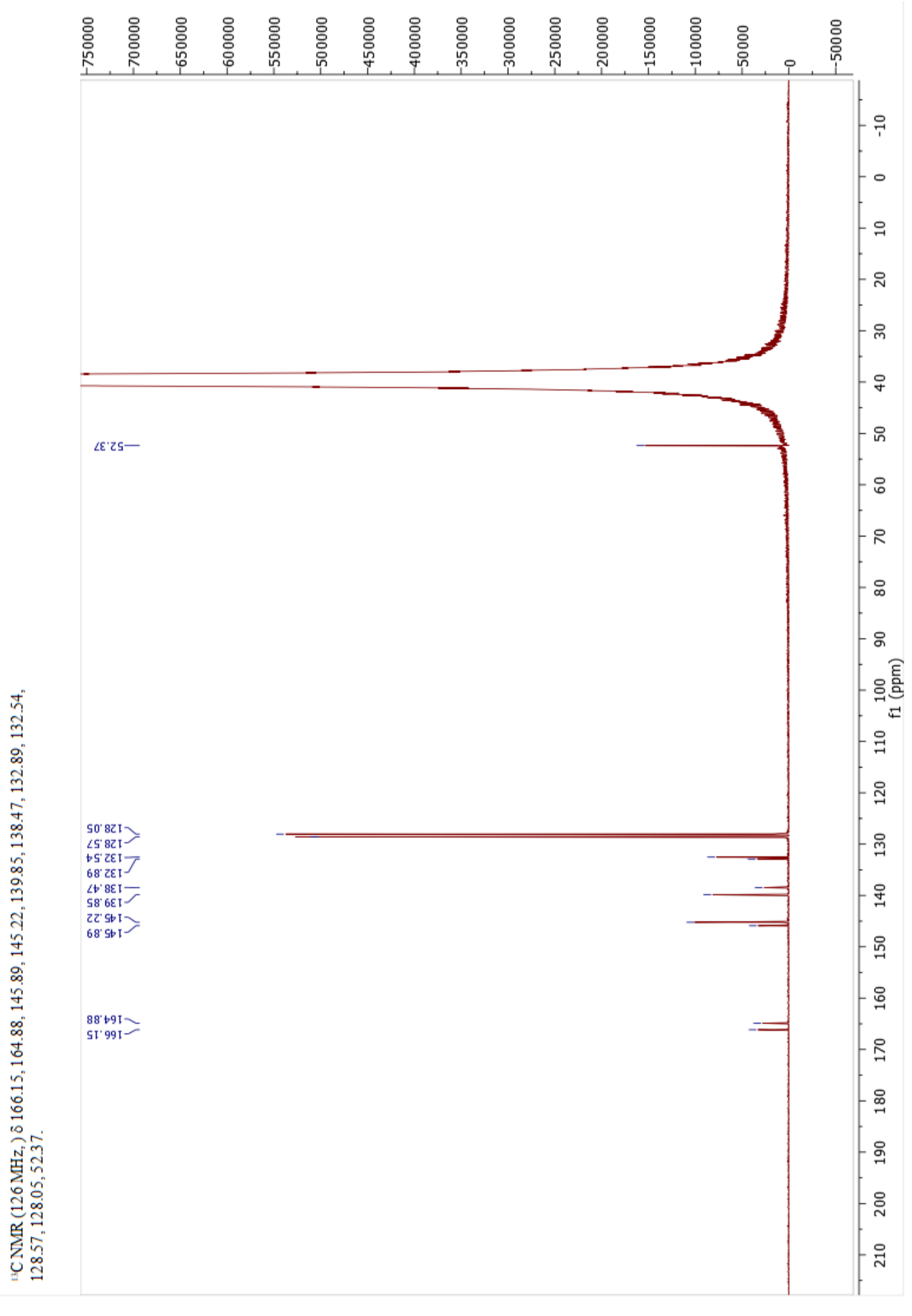


<smiles>O=C(Nc1nccnc1C(=O)O)c1ccccc1</smiles>

3-Benzamidopyrazine-2-carboxylic acid (5)

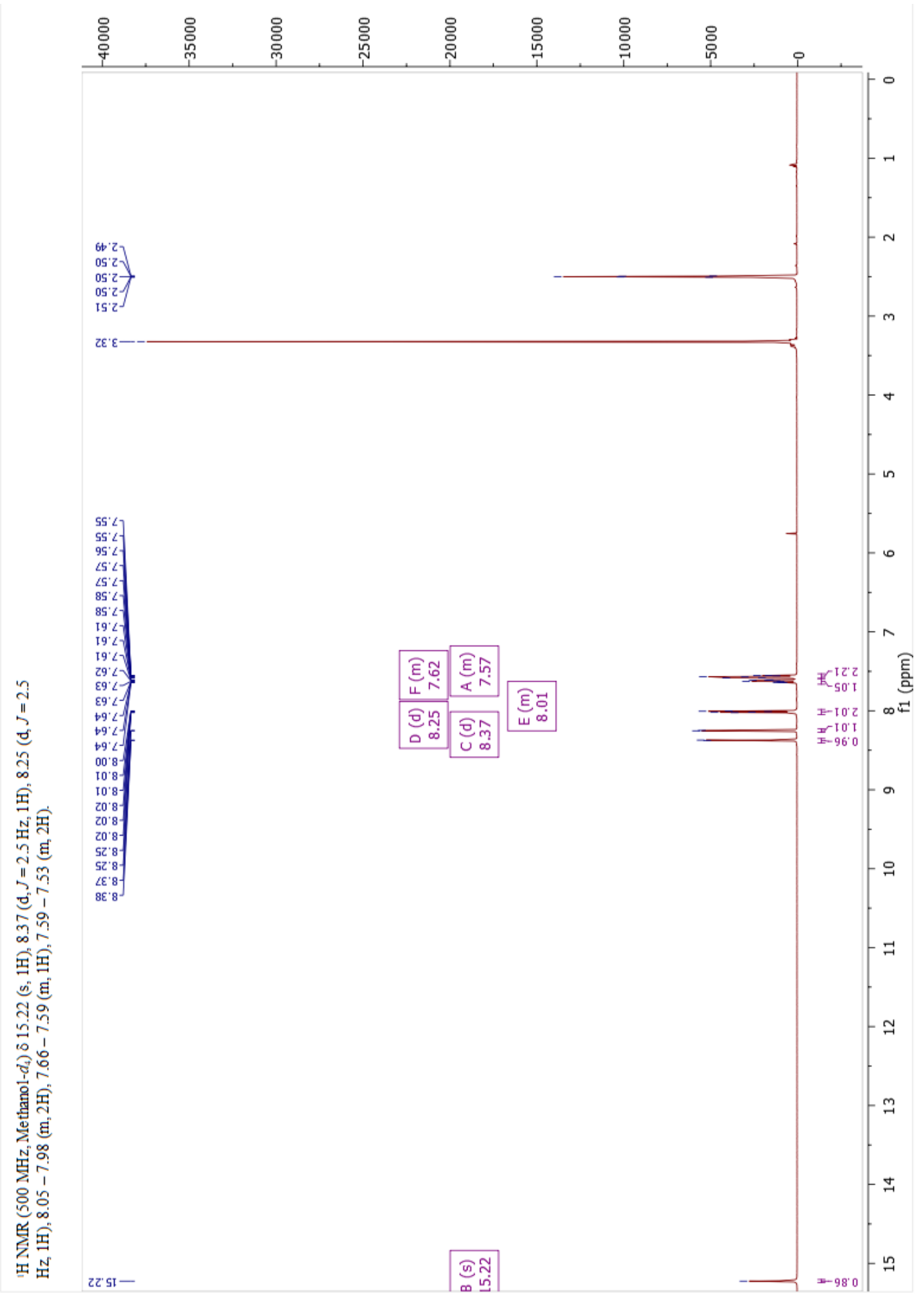




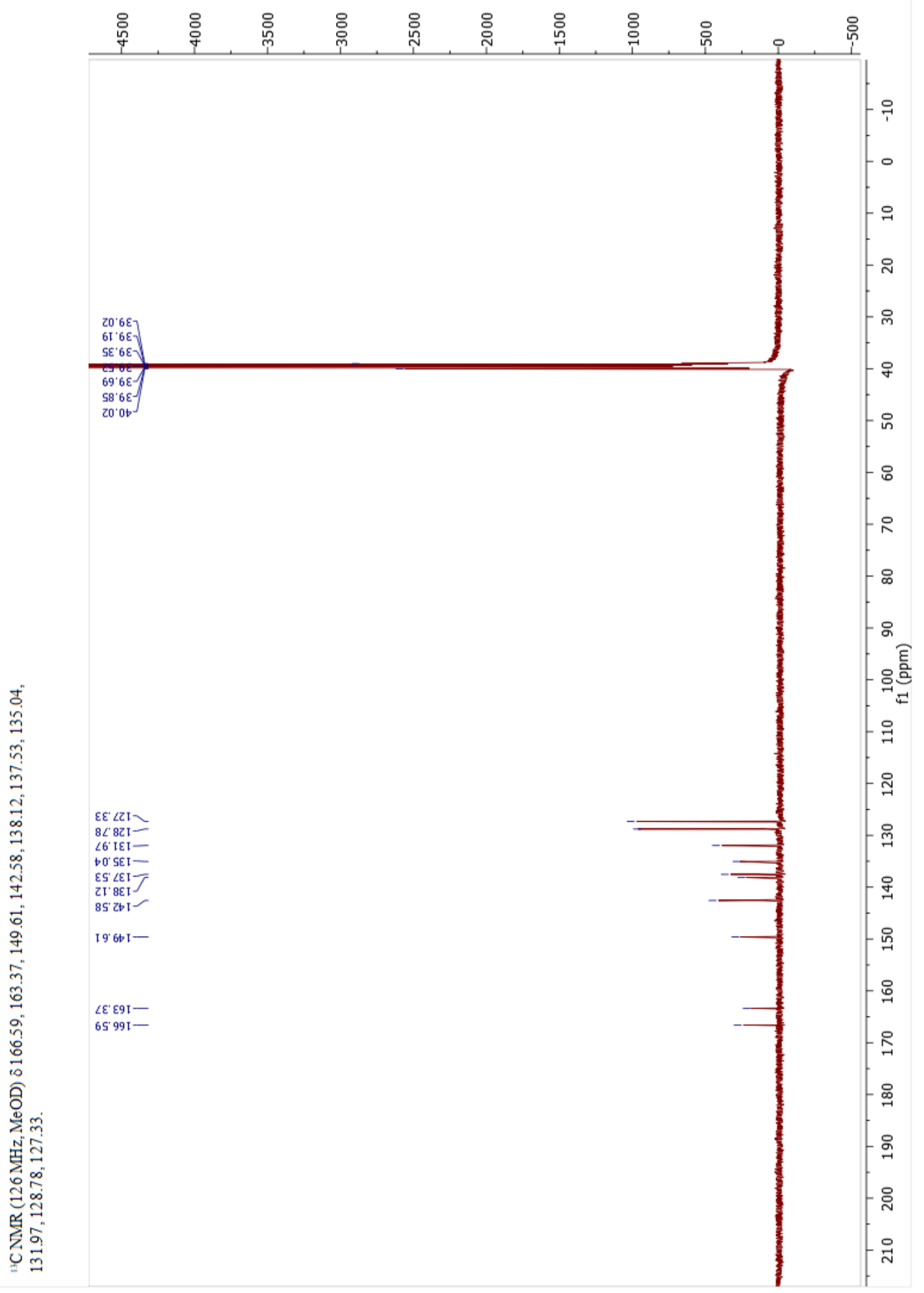


<smiles>Nc1ncc(I)nc1C(=O)O</smiles>

3-Amino-6-iodopyrazine-2-carboxylic acid (6)

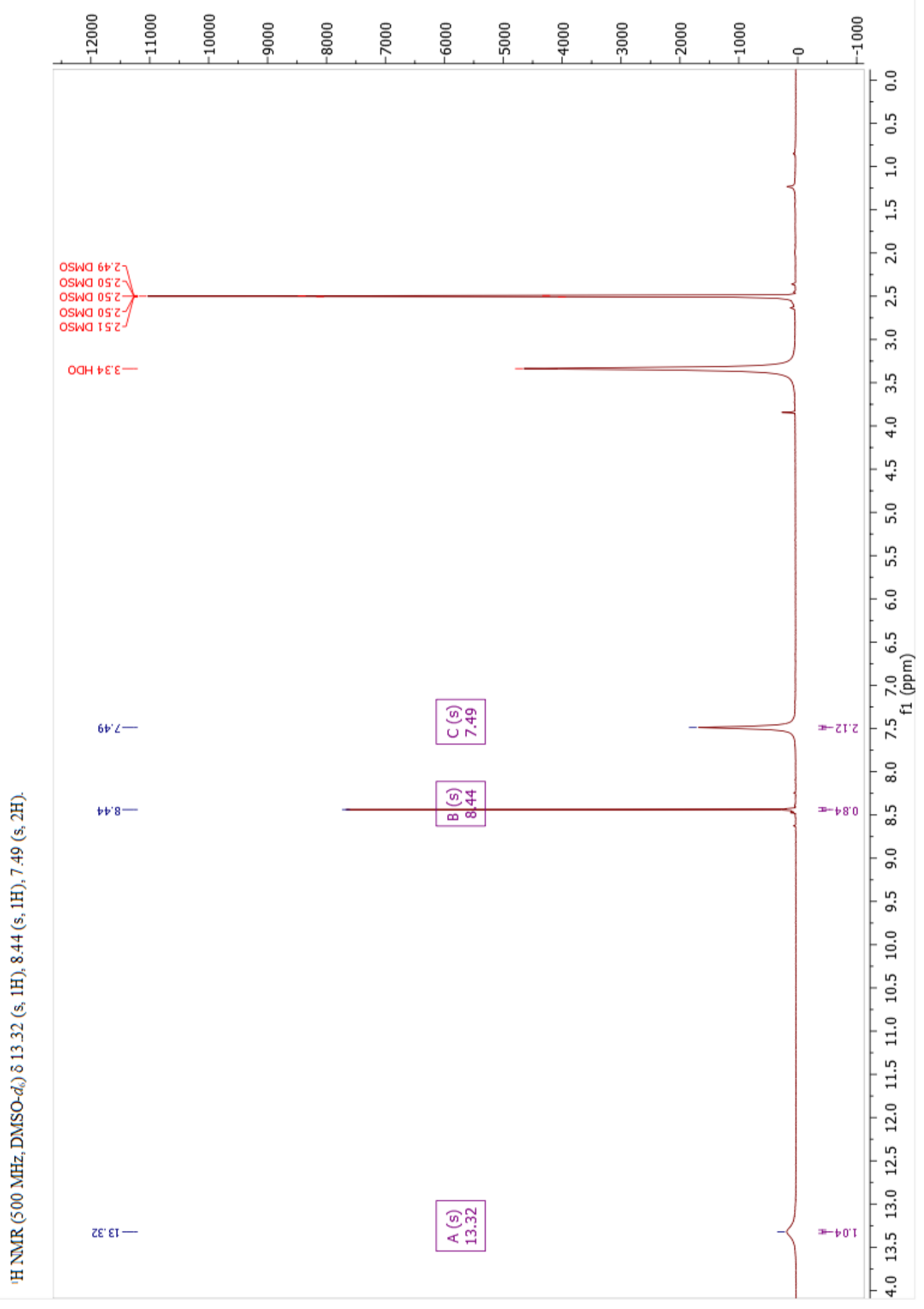


<smiles>CCC(=O)Nc1nccnc1C(=O)OC</smiles>

Methyl 3-propionamidopyrazine-2-carboxylate (7-OMe)

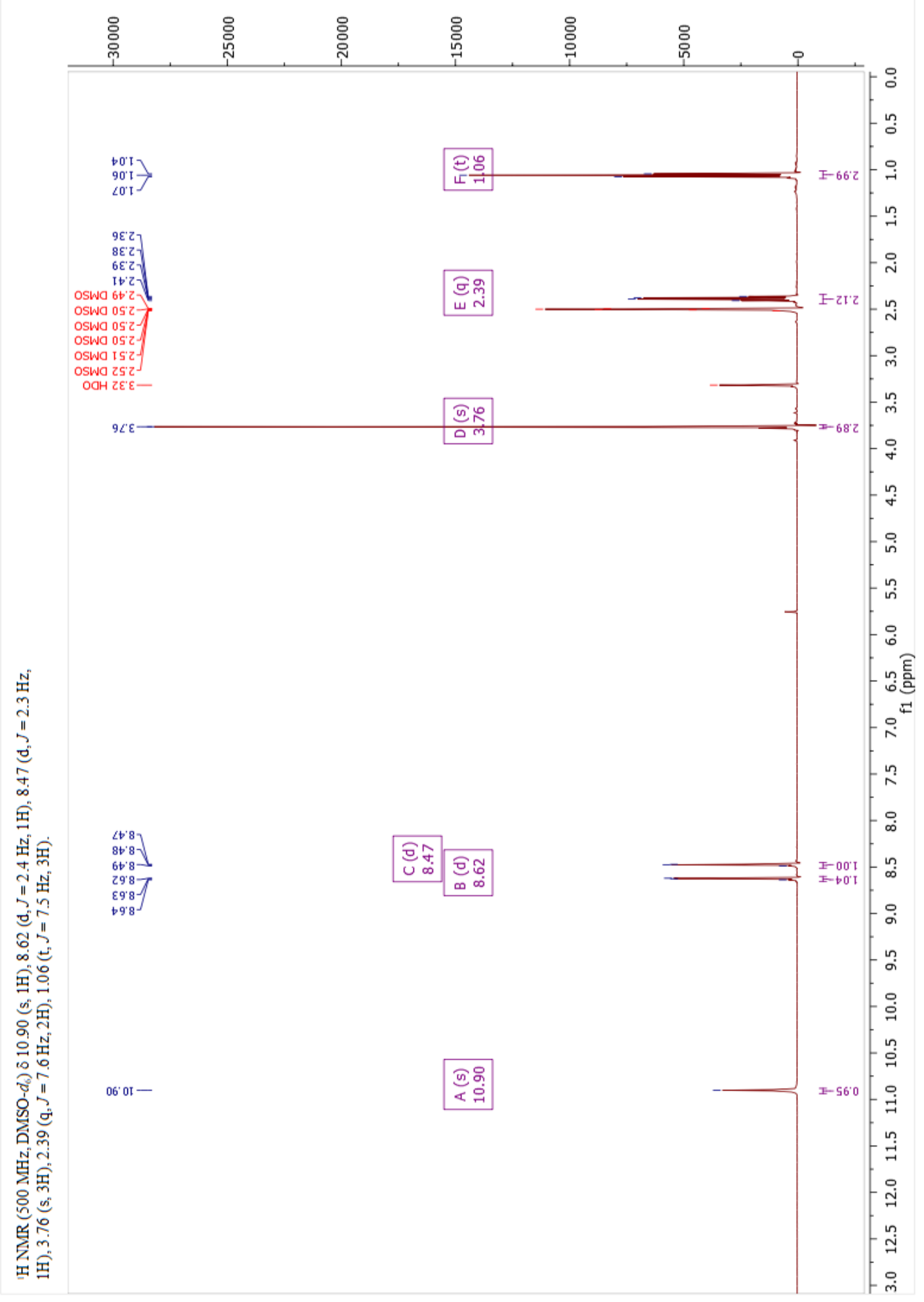




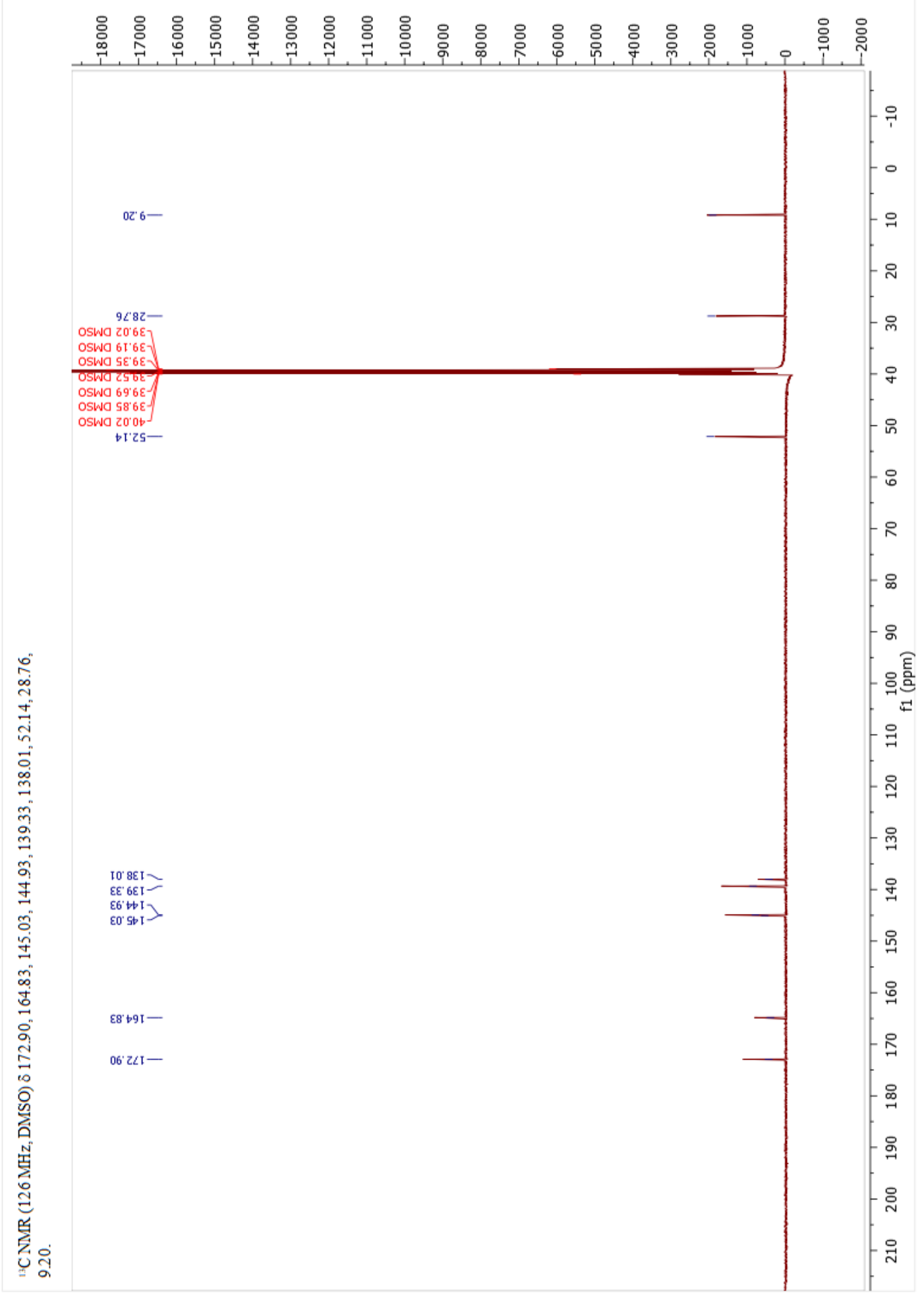




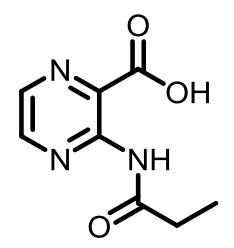

3-Propionamidopyrazine-2-carboxylic acid (7)

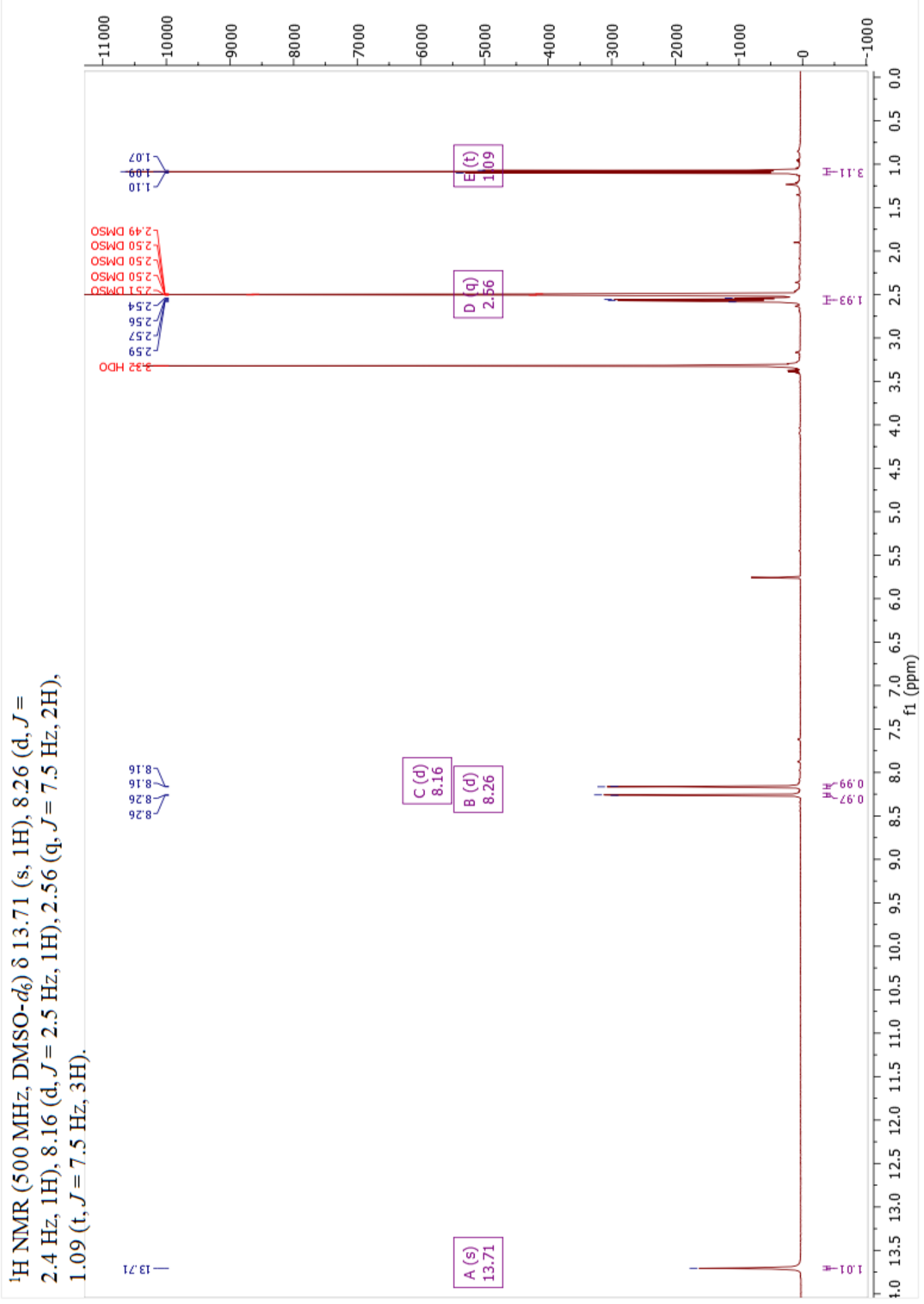




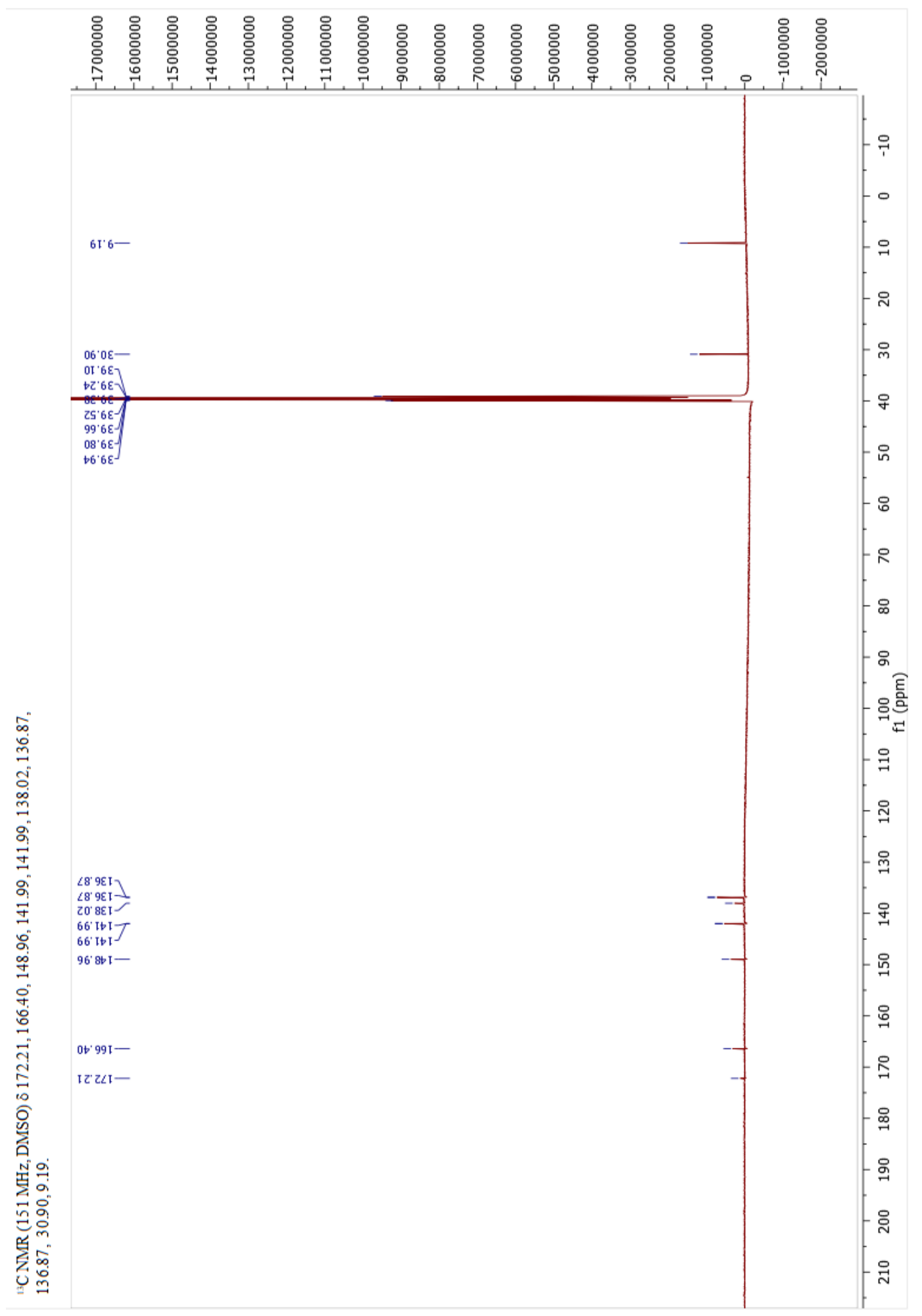


<smiles>CCNc1nccnc1C(=O)OC</smiles>

Methyl 3-(ethylamino)pyrazine-2-carboxylate (8-OMe)

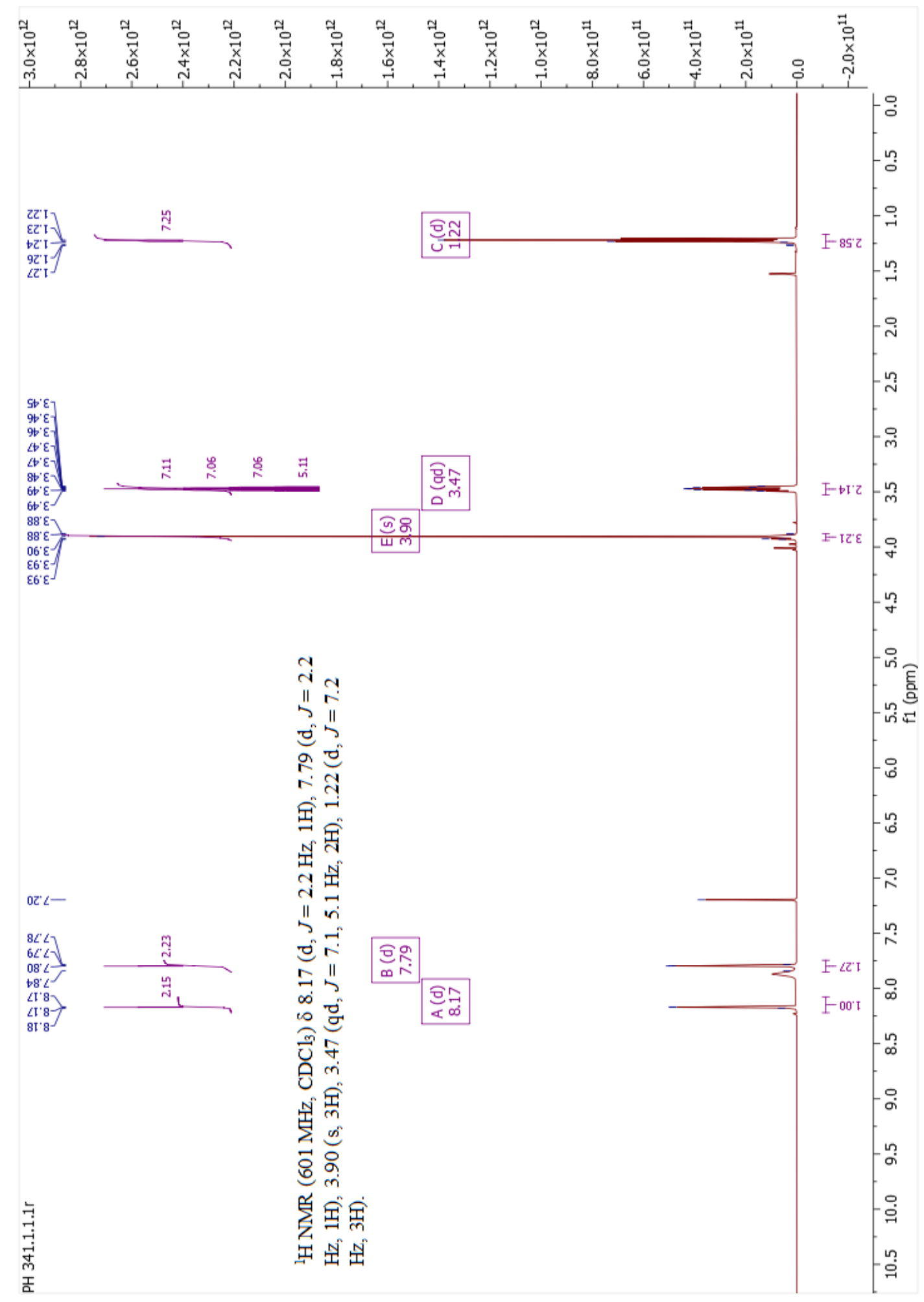




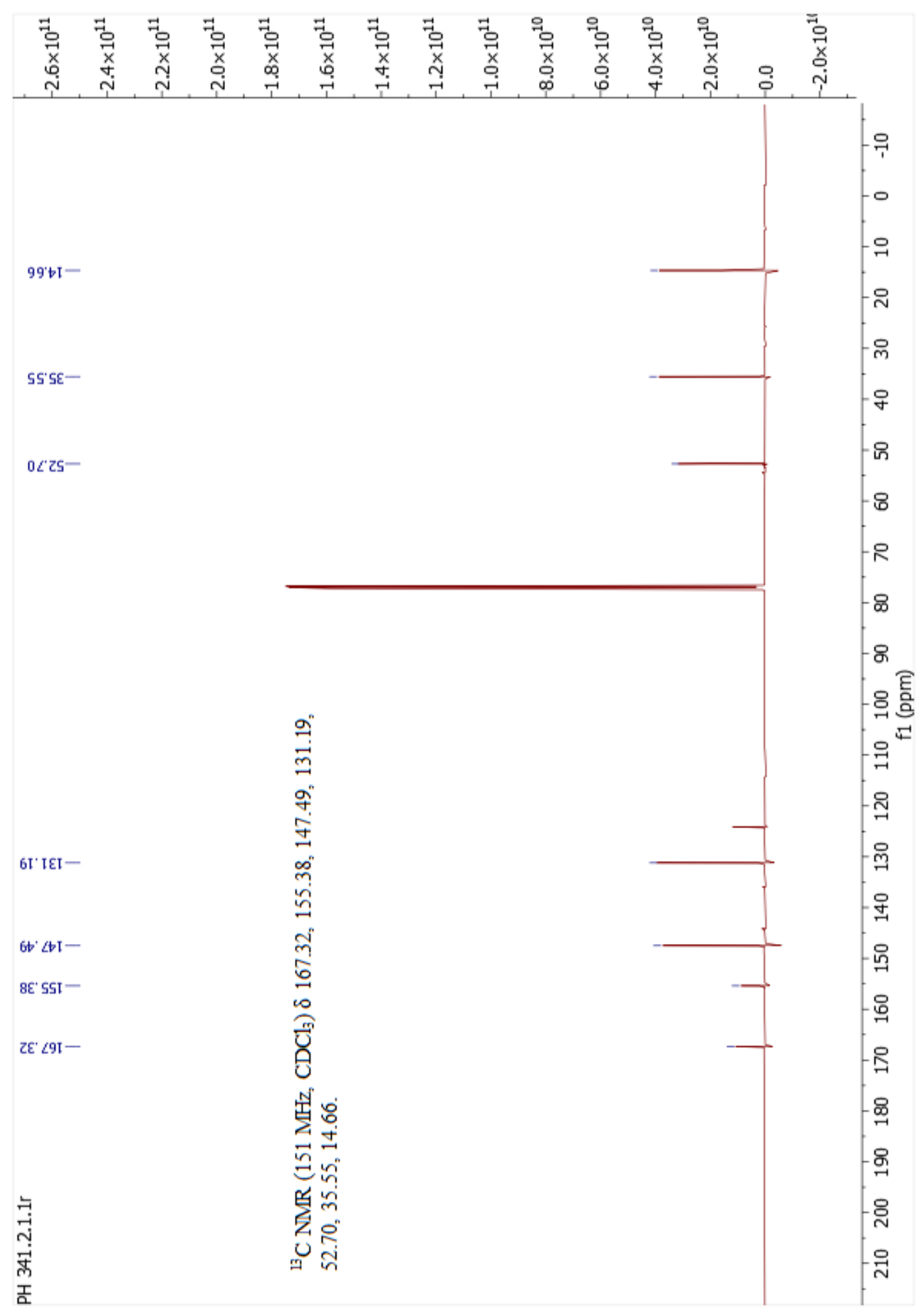




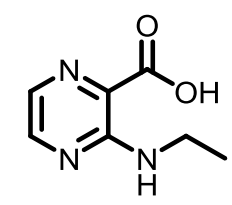

3-(ethylamino)pyrazine-2-carboxylic acid (8)

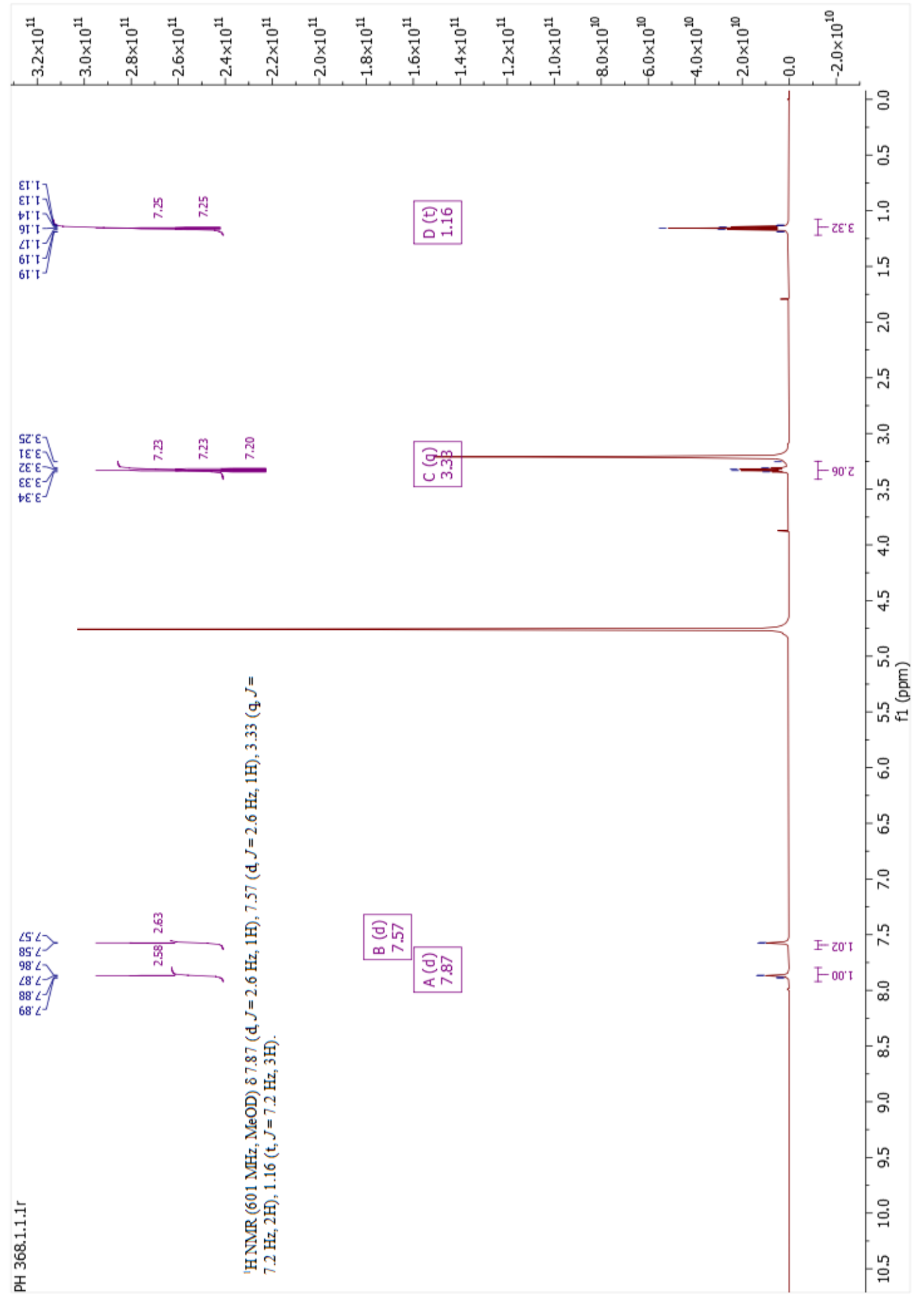




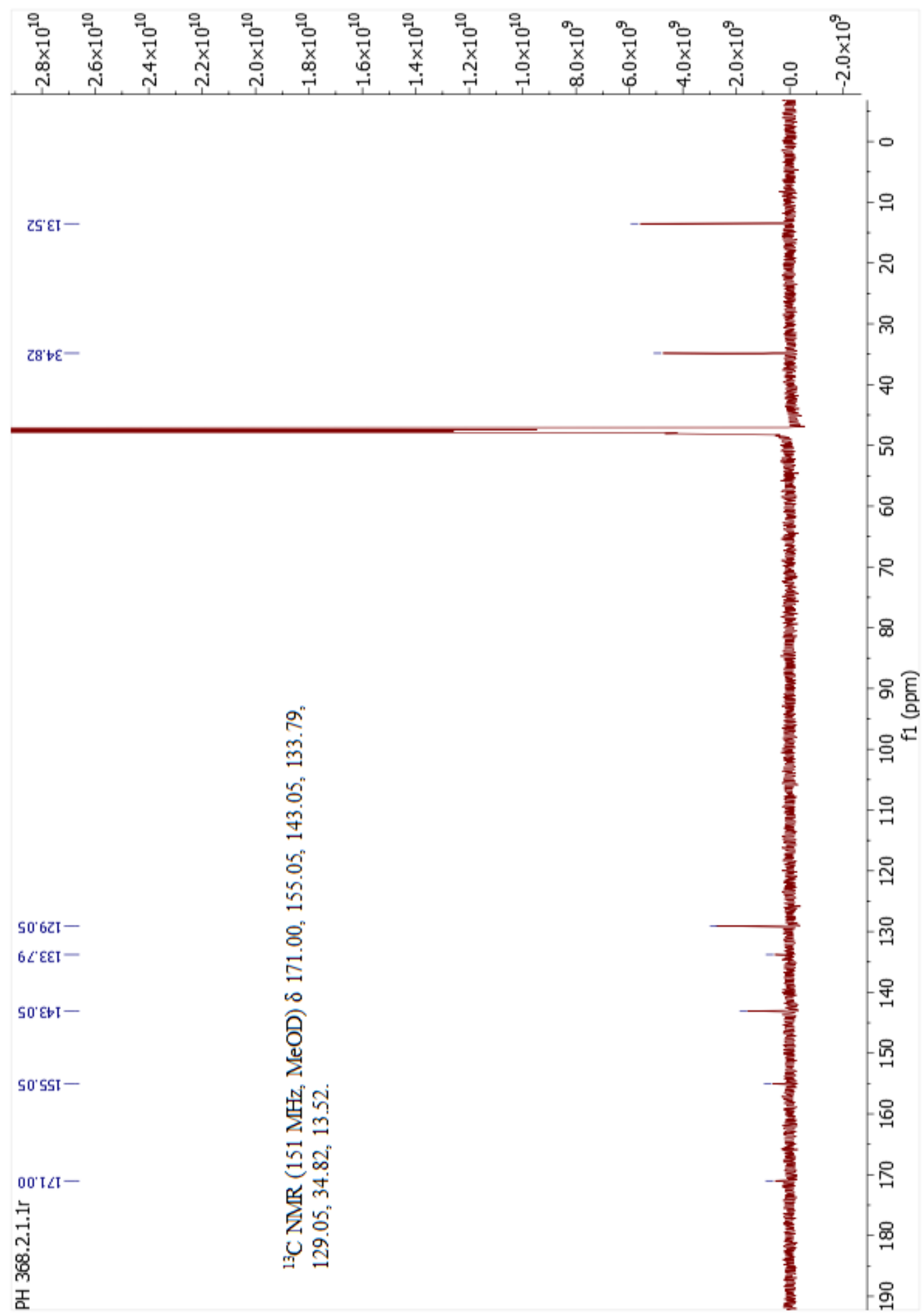

S50 


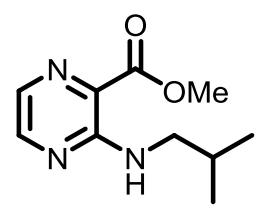

Methyl 3-(isobutylamino)pyrazine-2-carboxylate (9-OMe)

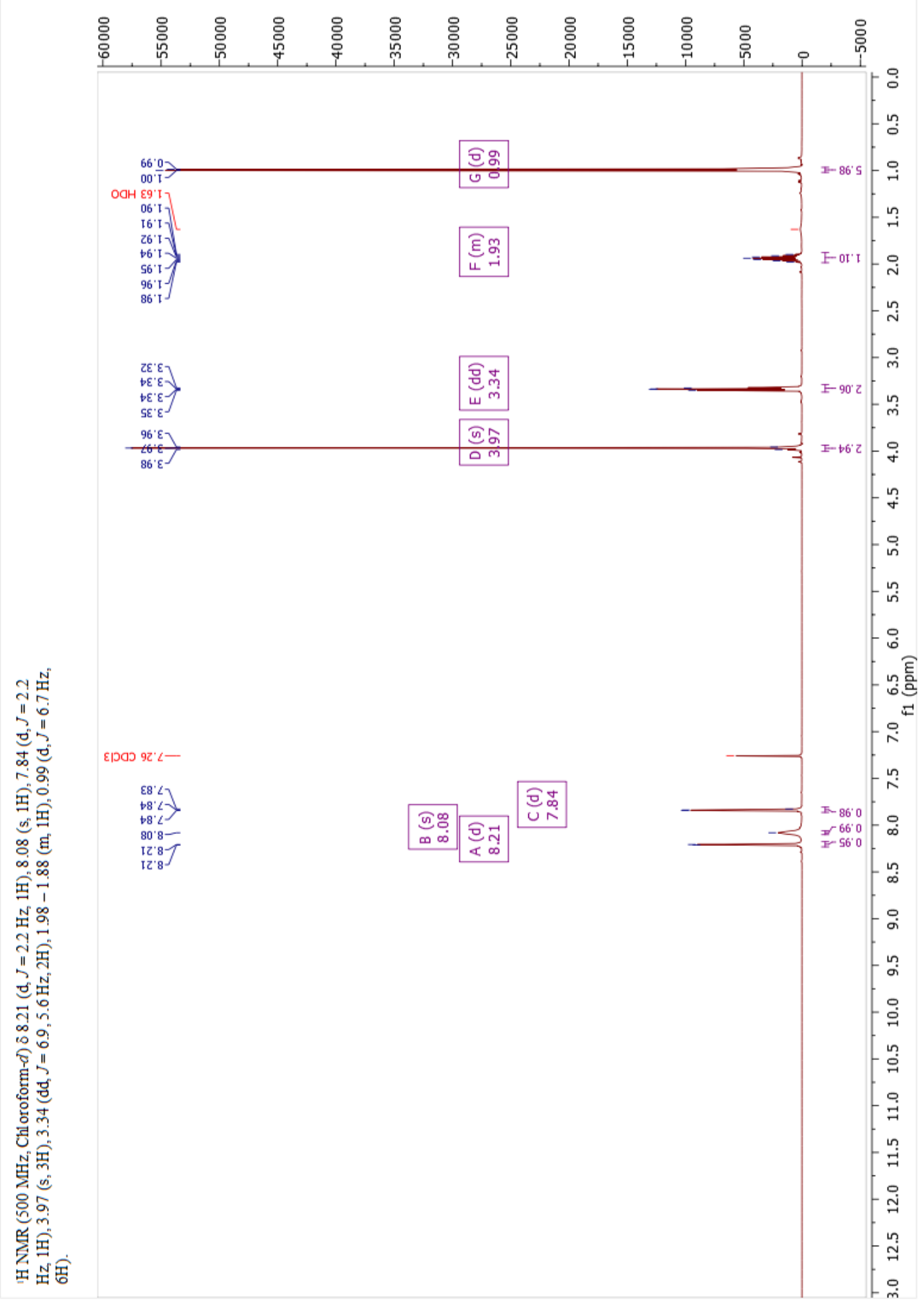




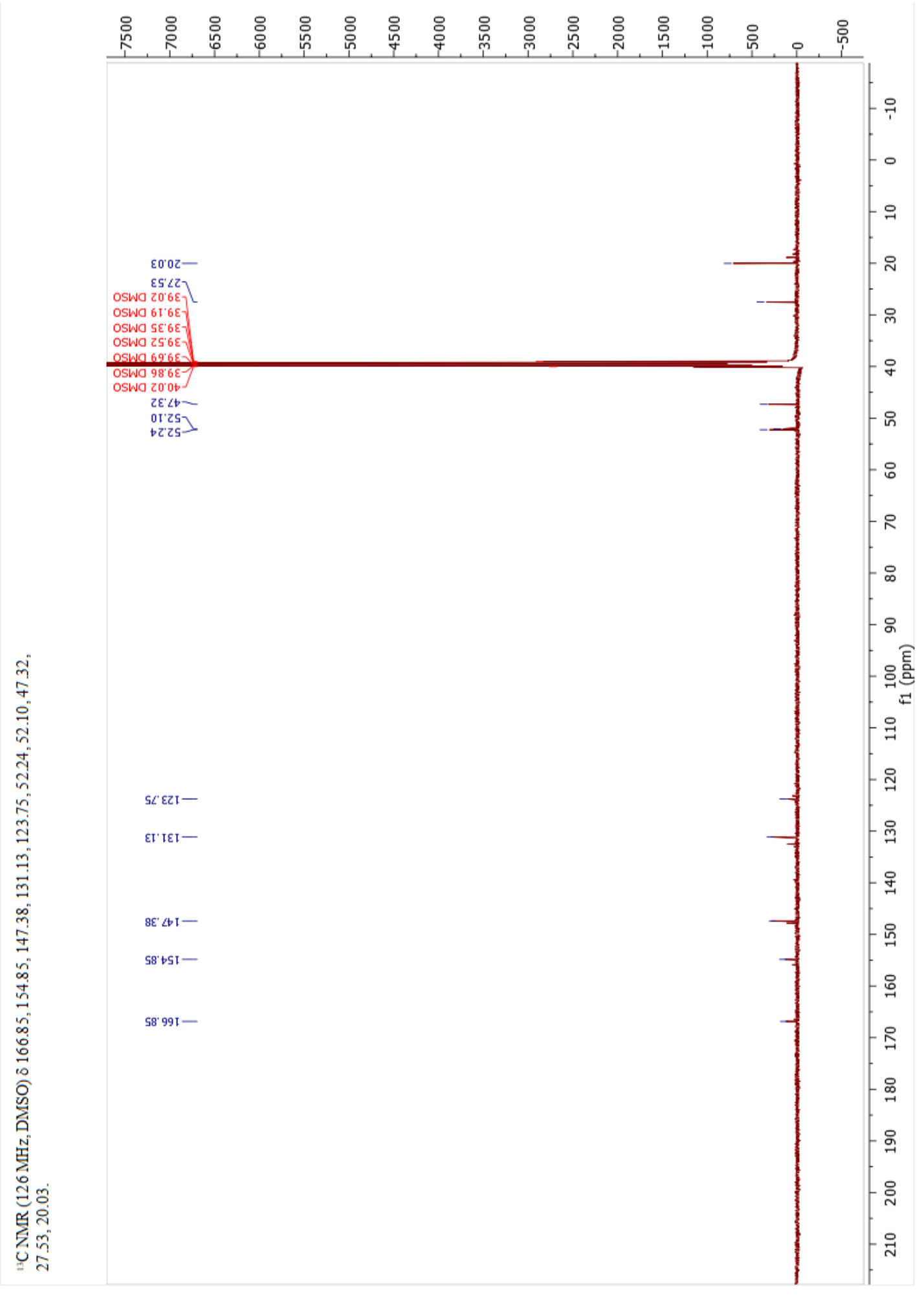


<smiles>CC(C)CNc1nccnc1C(=O)O</smiles>

3-(Isobutylamino)pyrazine-2-carboxylic acid (9)

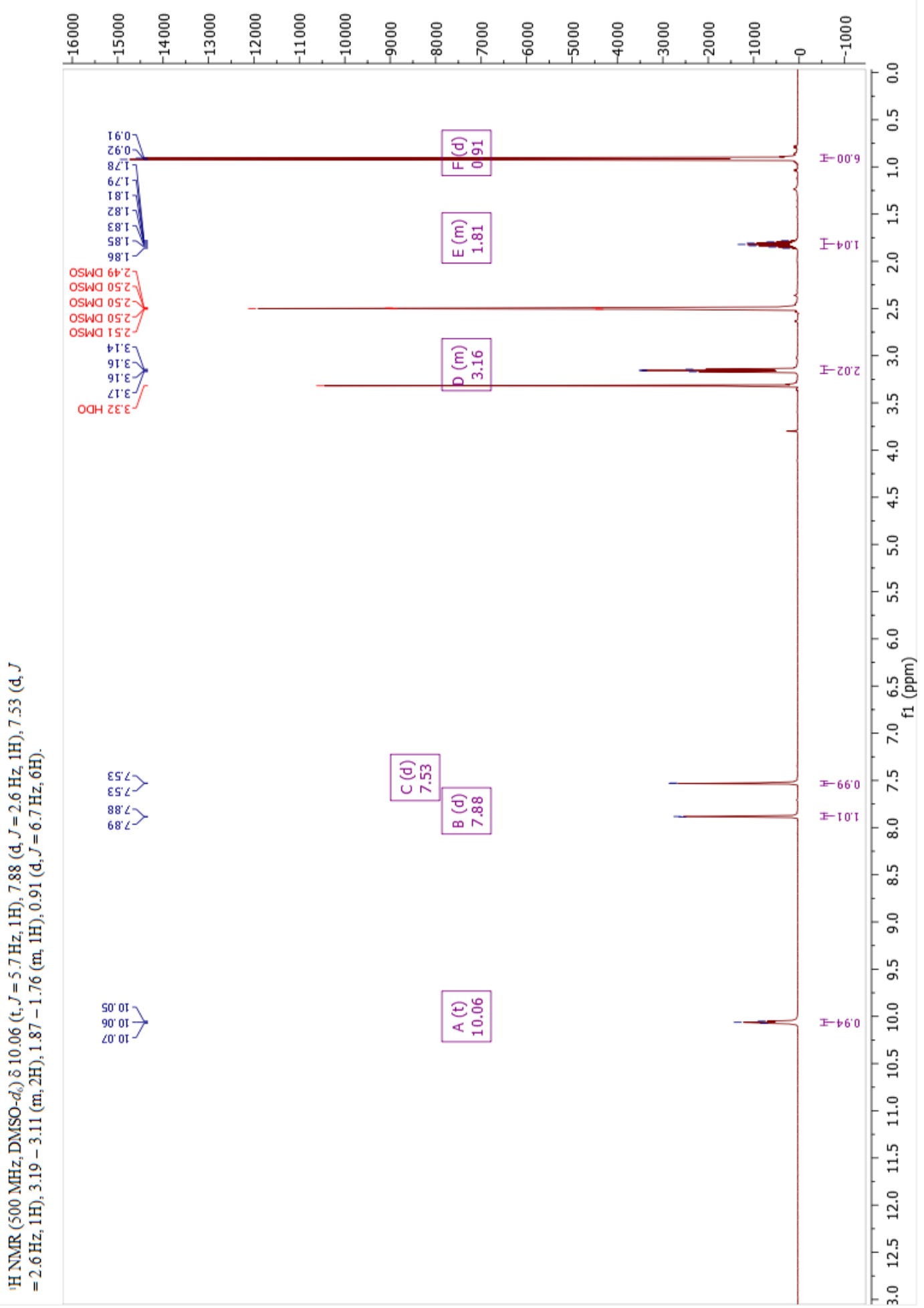




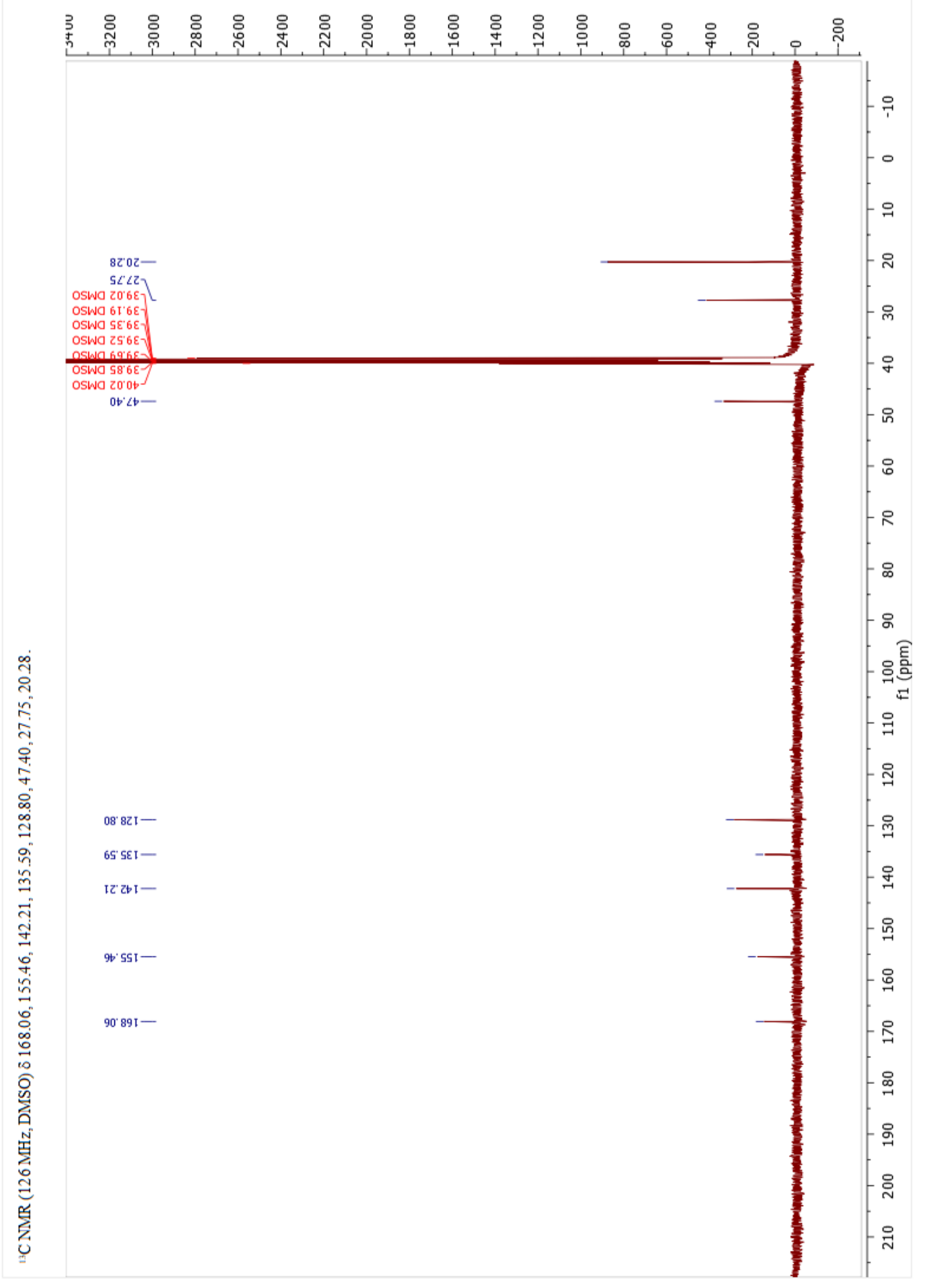




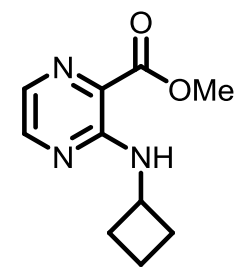

Methyl 3-(cyclobutylamino)pyrazine-2-carboxylate (10-OMe)

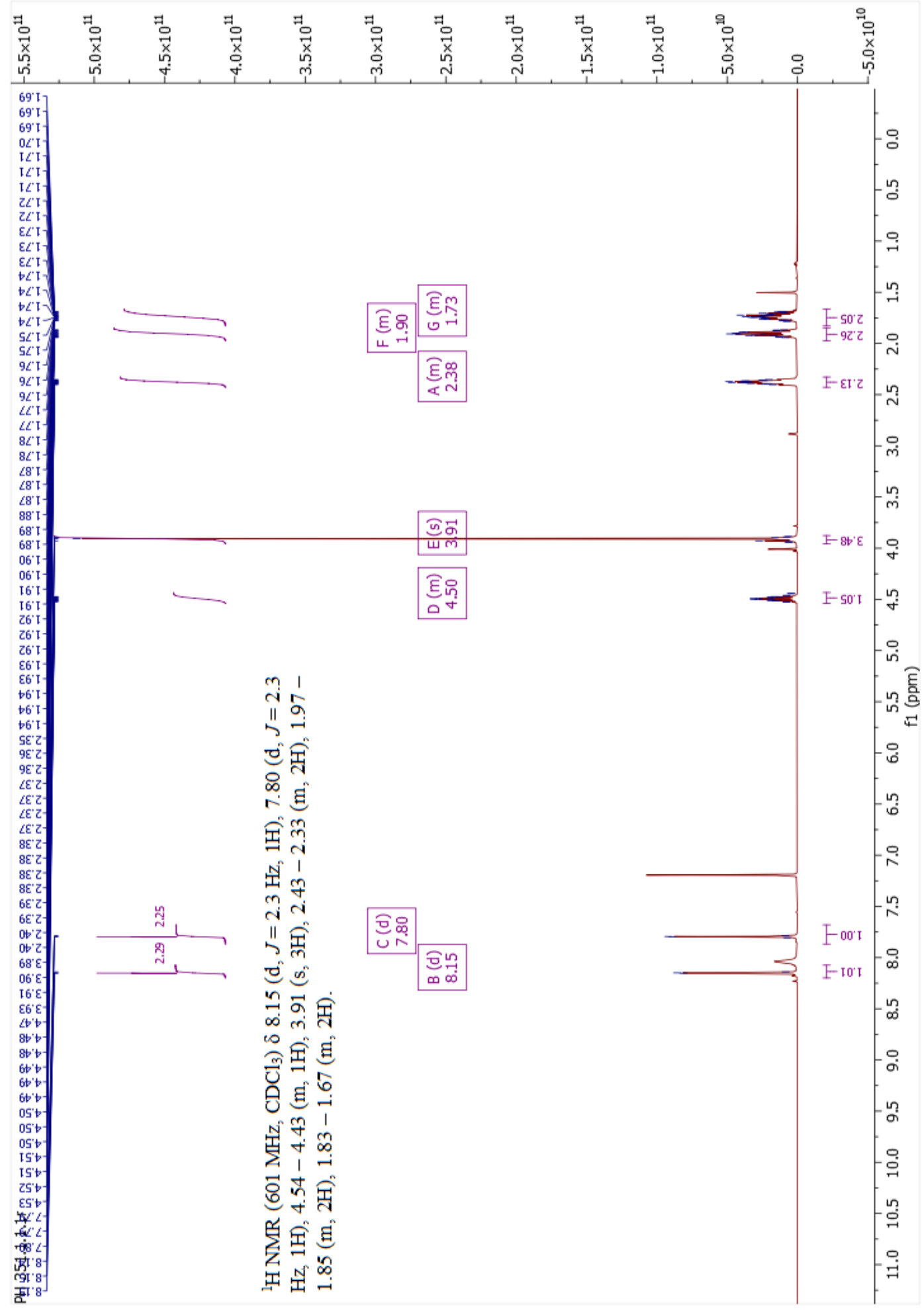




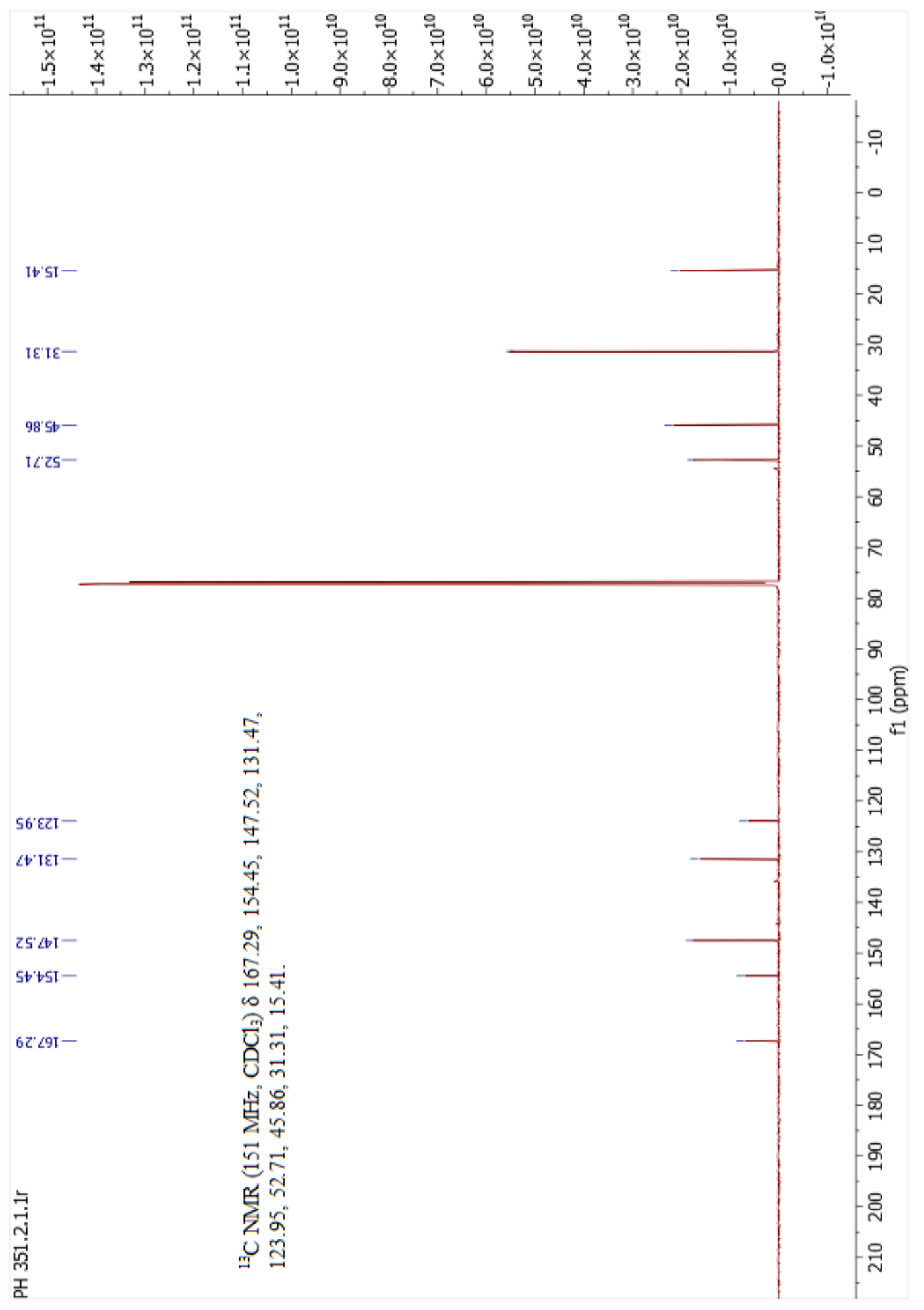




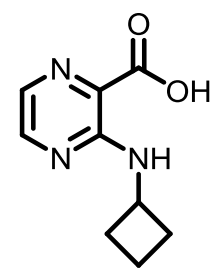

3-(Cyclobutylamino)pyrazine-2-carboxylic acid (10)

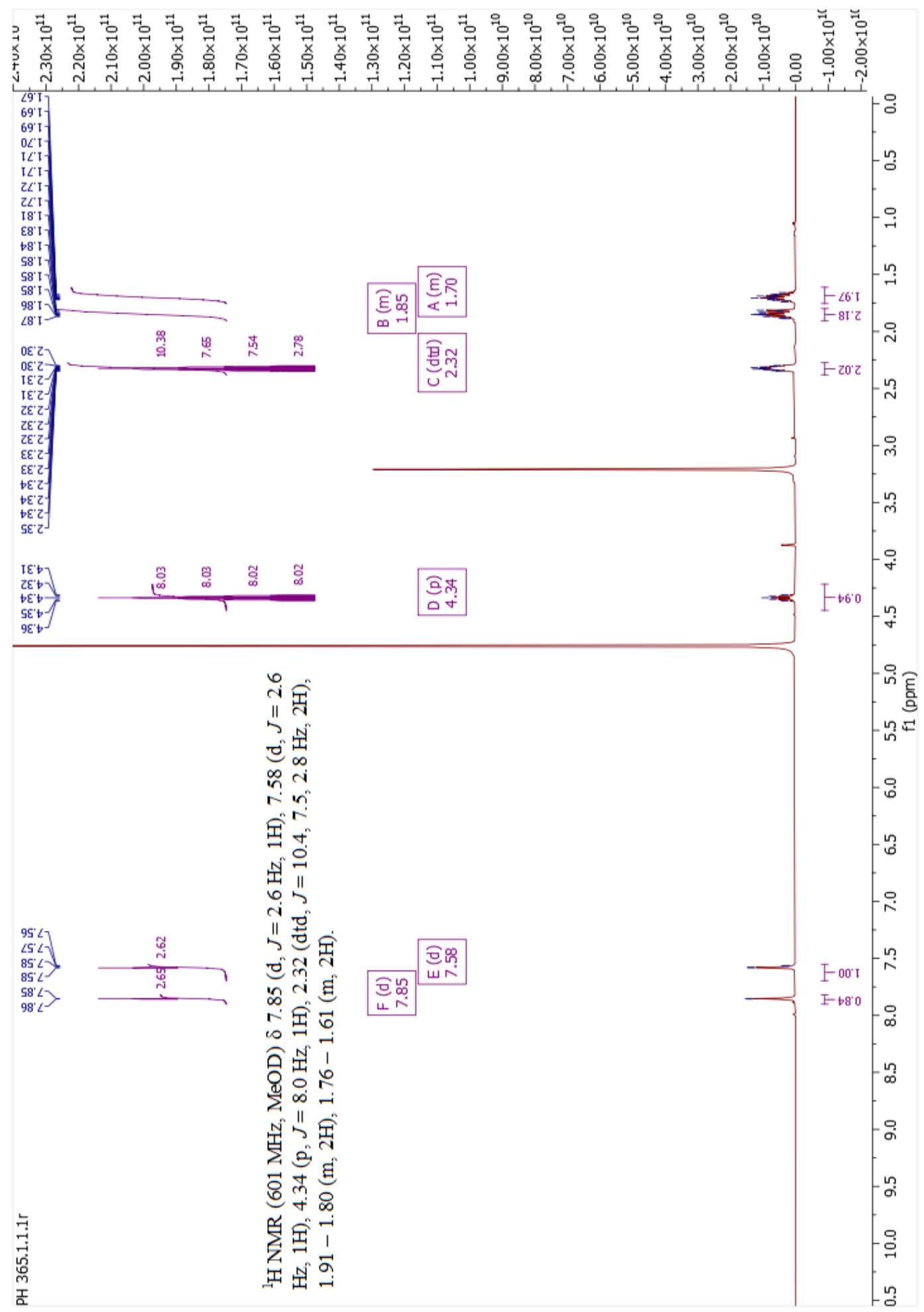




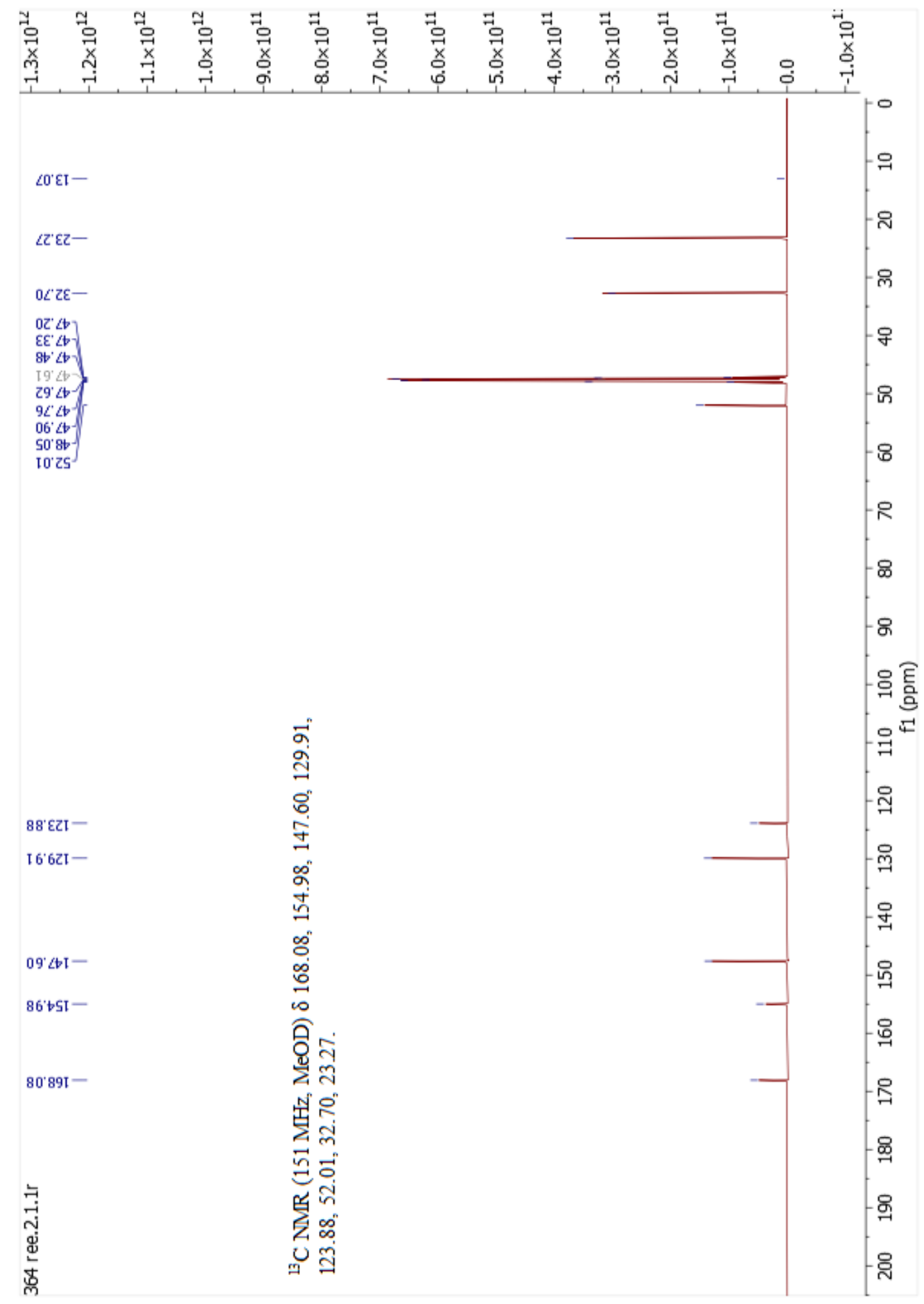




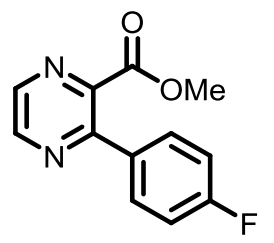

Methyl 3-(4-fluorophenyl)pyrazine-2-carboxylate (11-OMe)

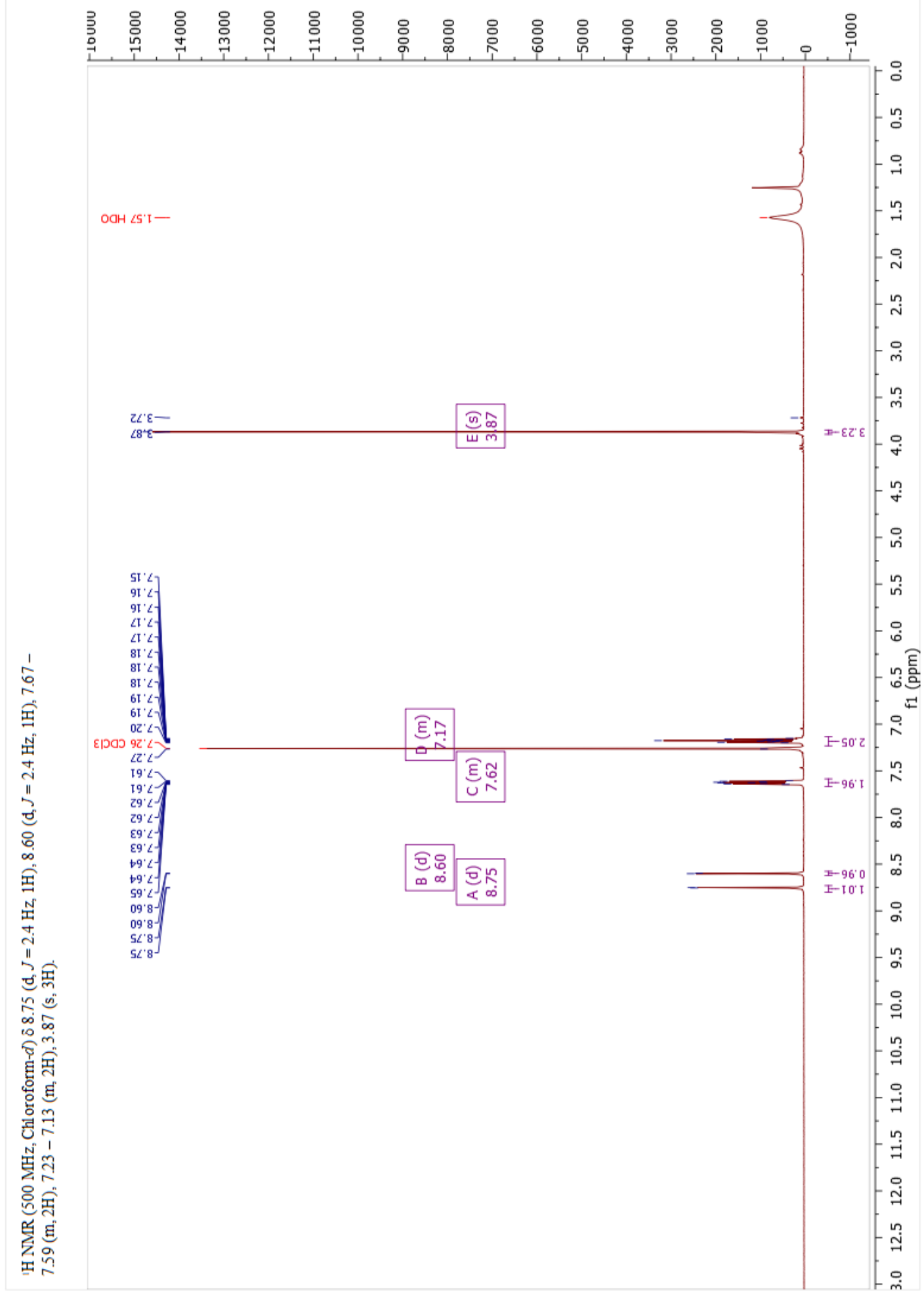




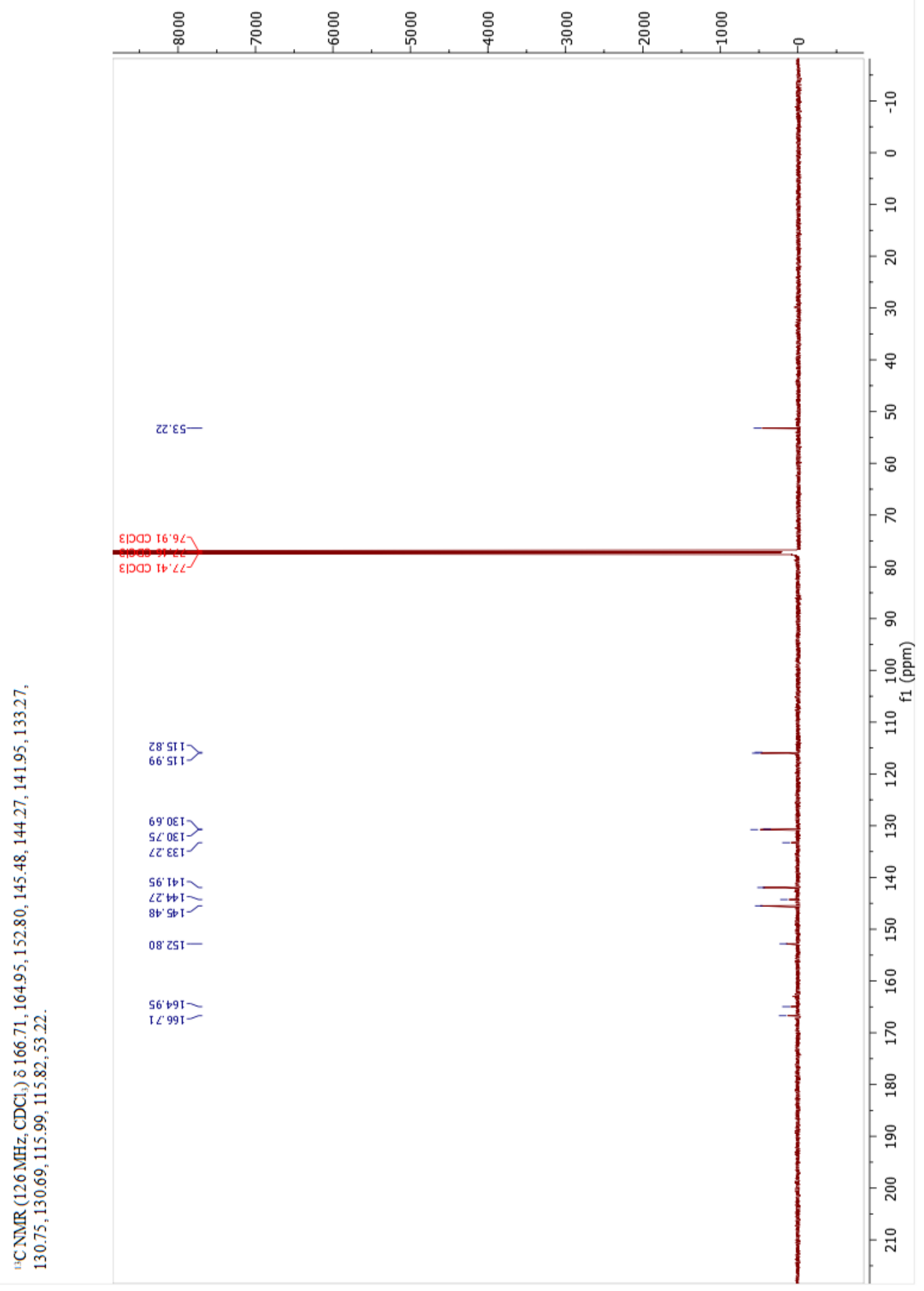




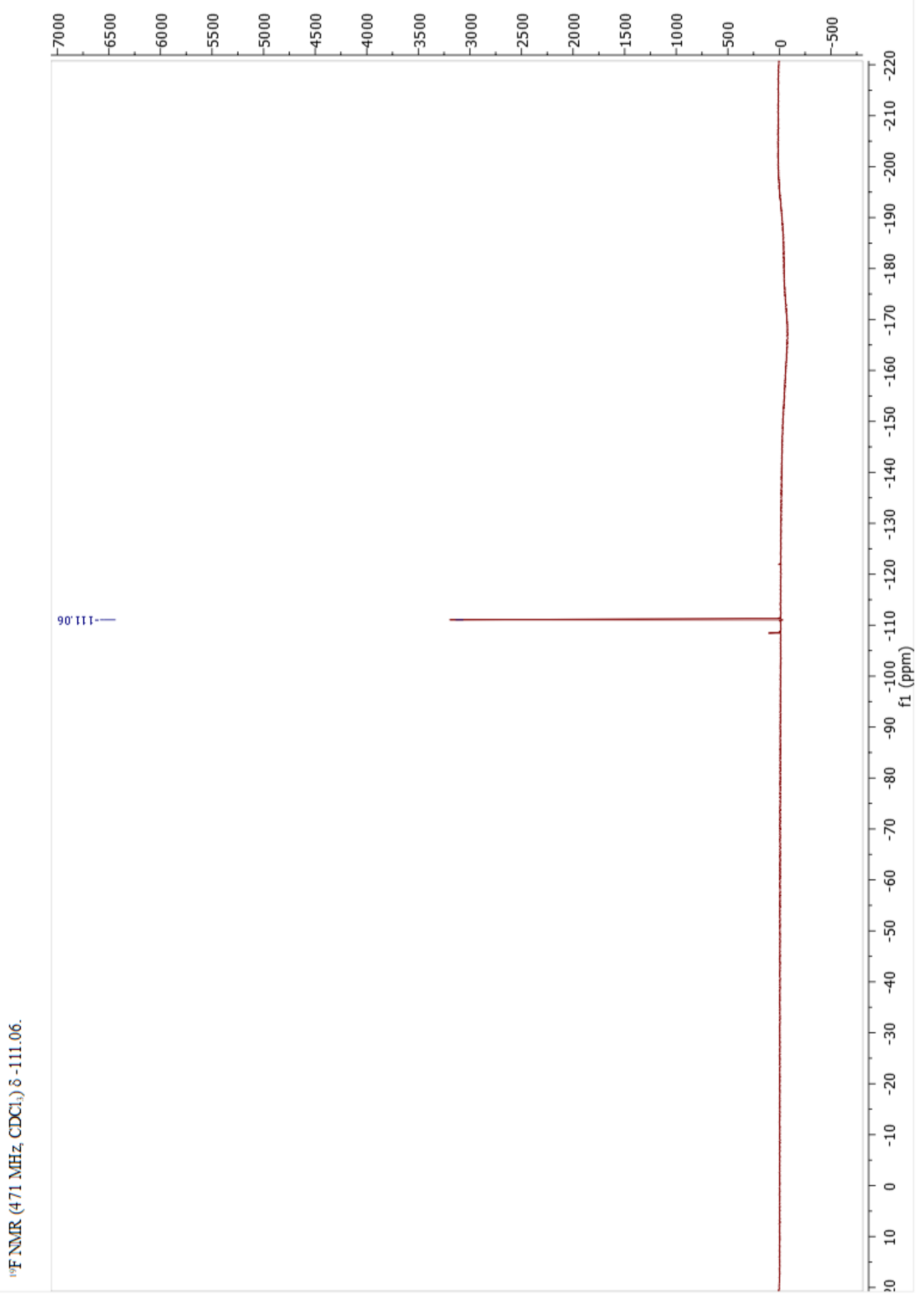




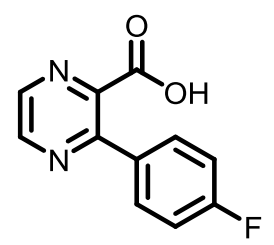

3-(4-Fluorophenyl)pyrazine-2-carboxylic acid (11)

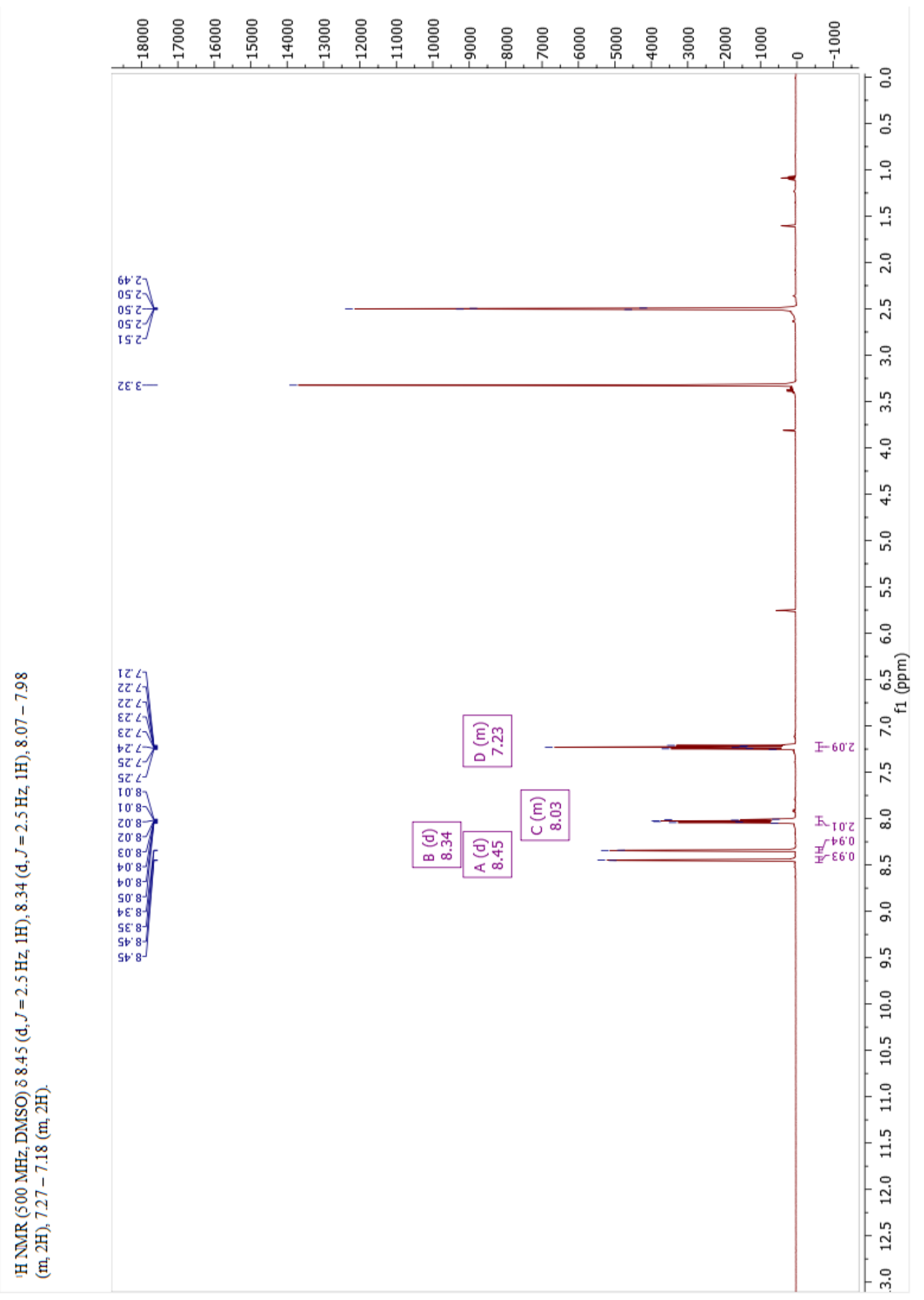




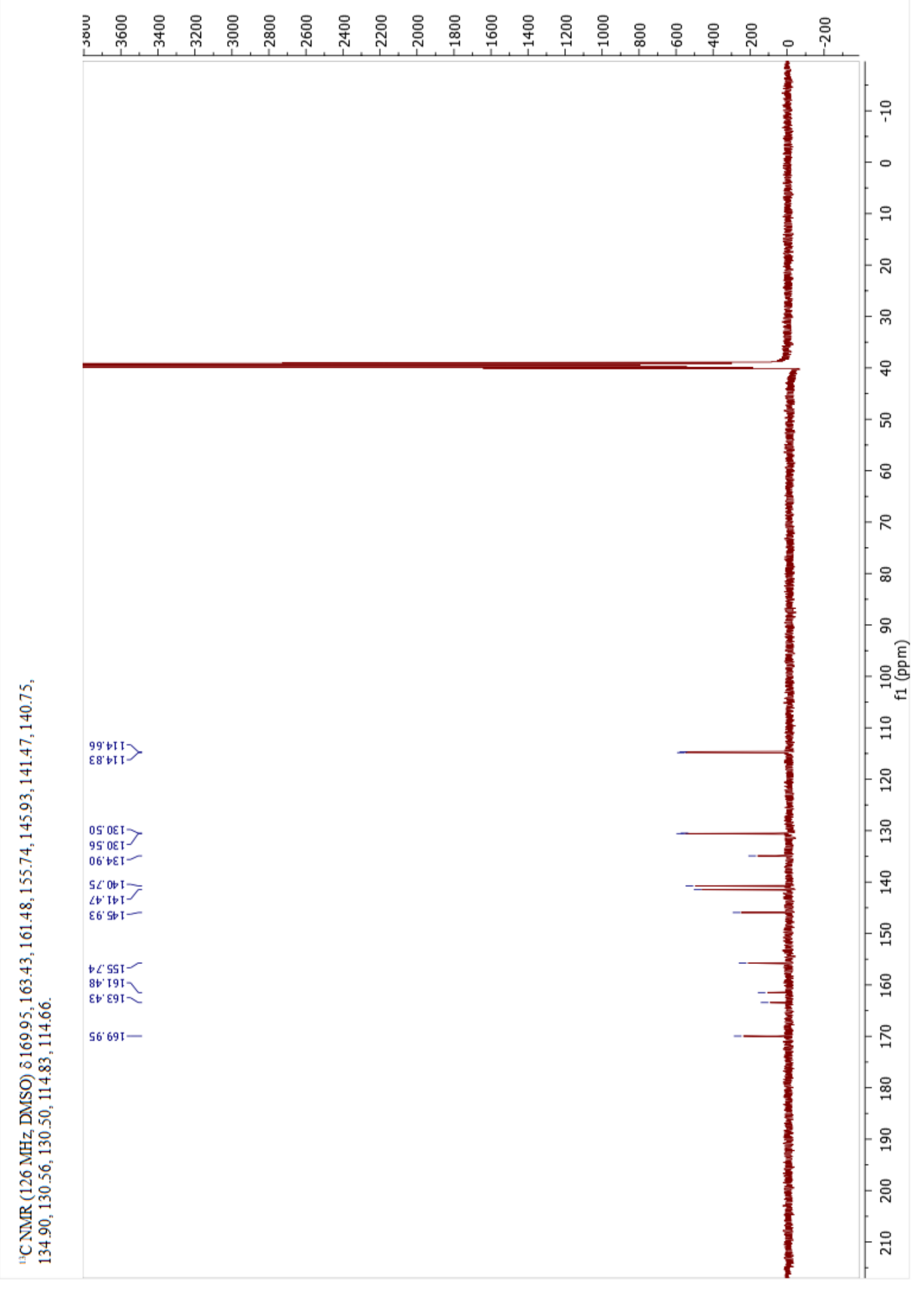




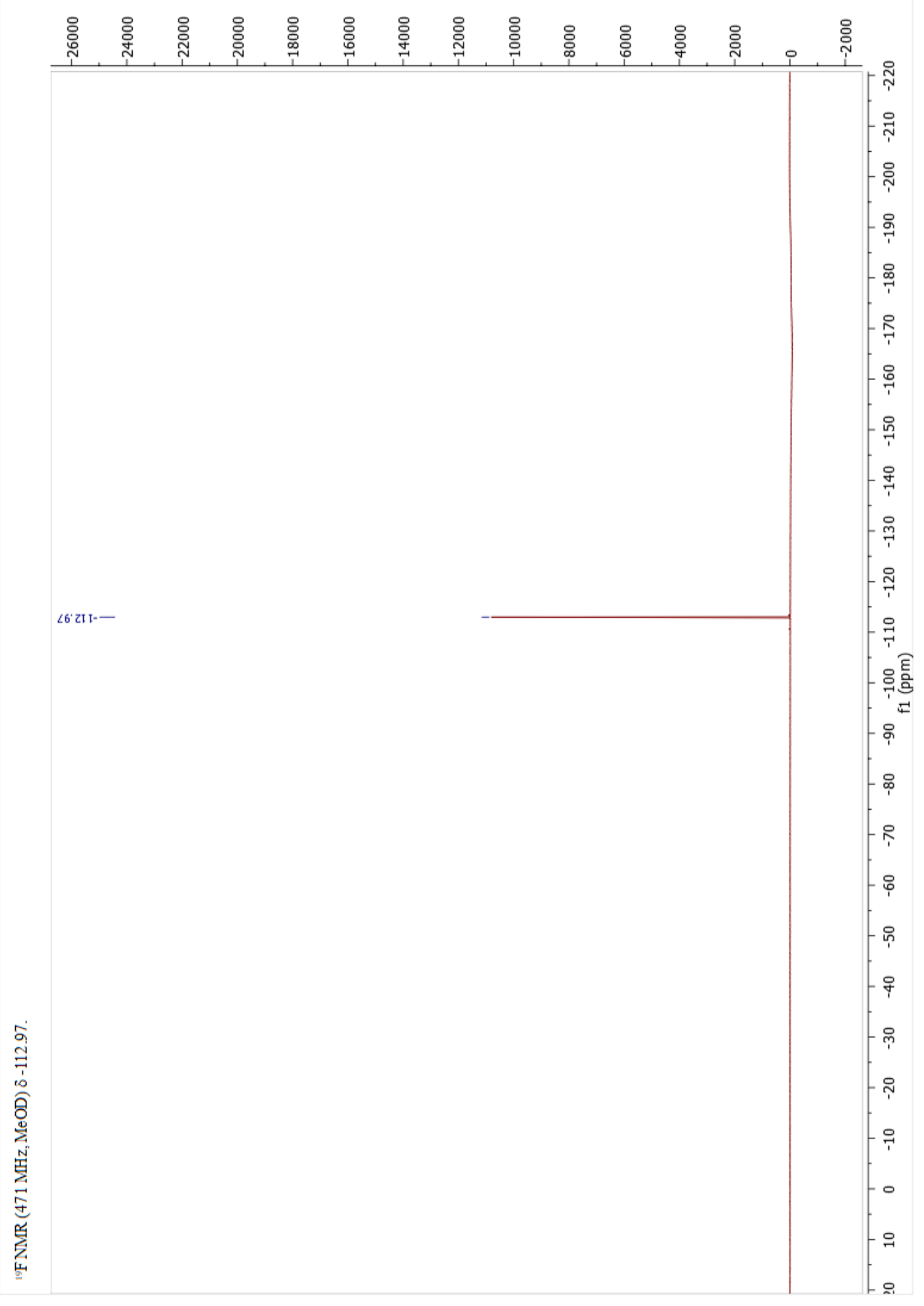




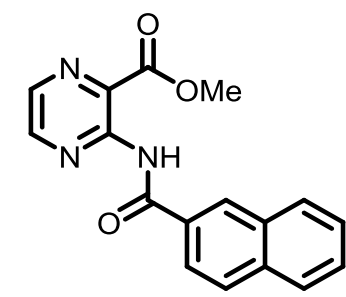

Methyl 3-(2-naphthamido)pyrazine-2-carboxylate (12-OMe)

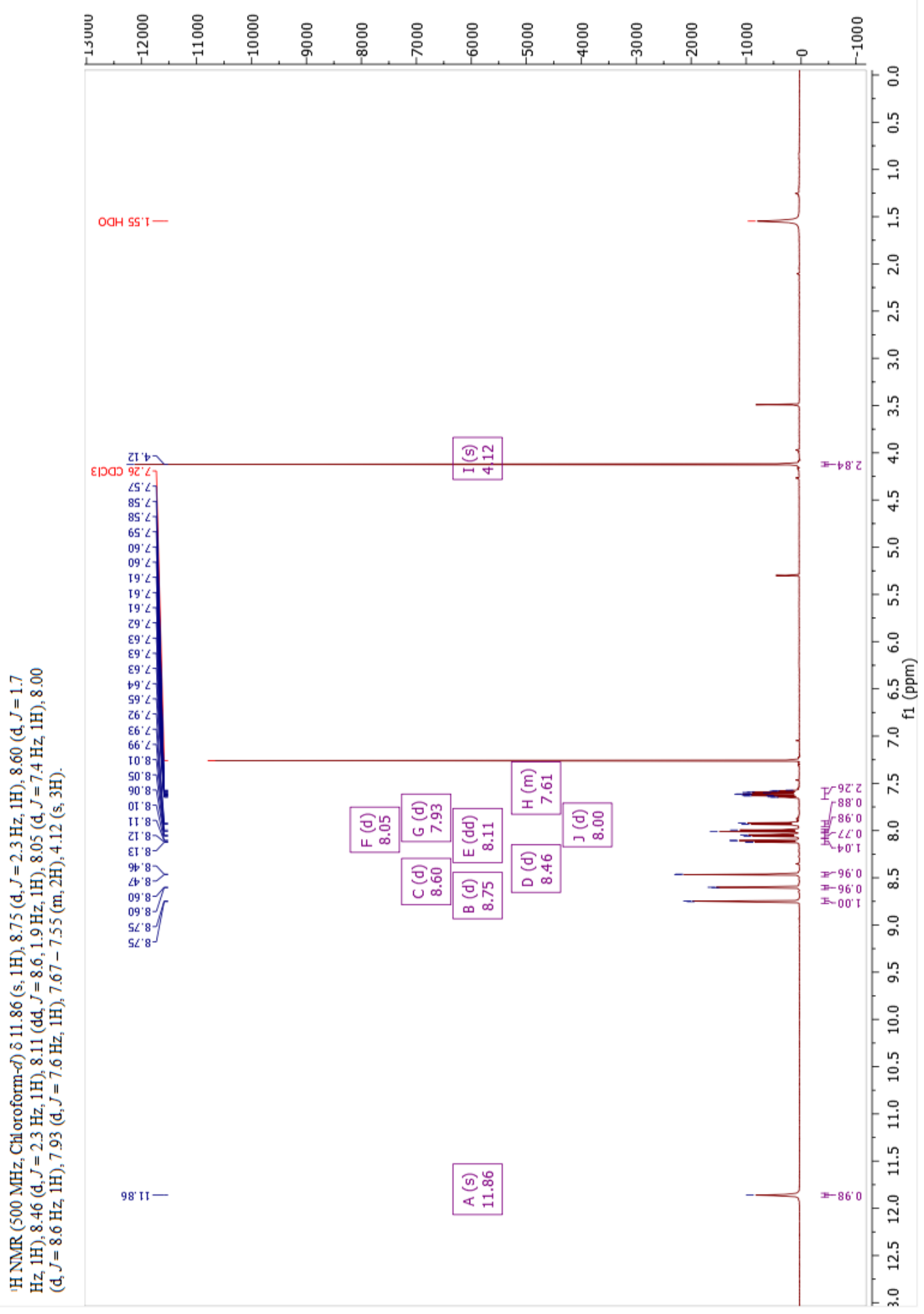




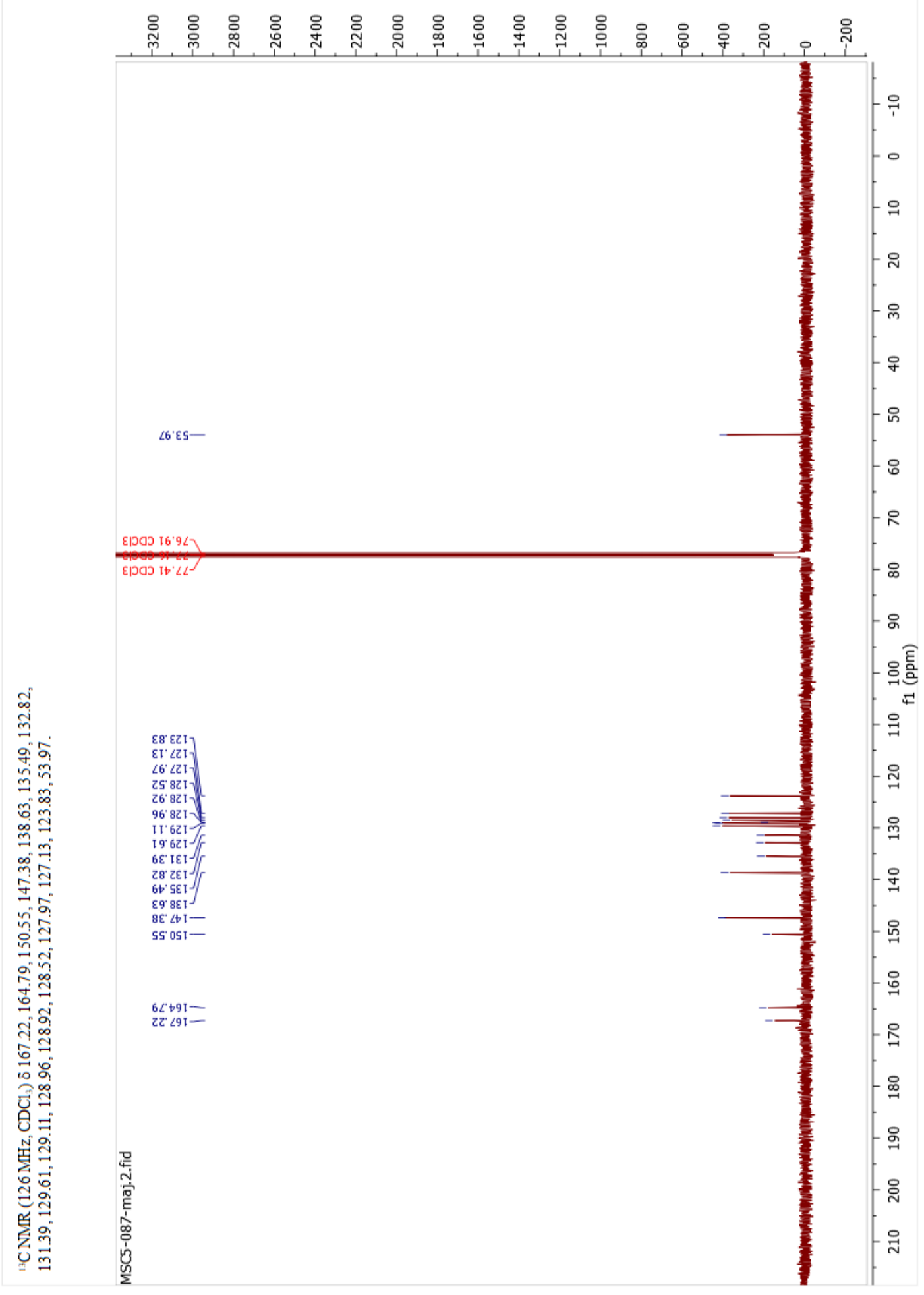


<smiles>O=C(Nc1nccnc1C(=O)O)c1ccc2ccccc2c1</smiles>

3-(2-Naphthamido)pyrazine-2-carboxylic acid (12)

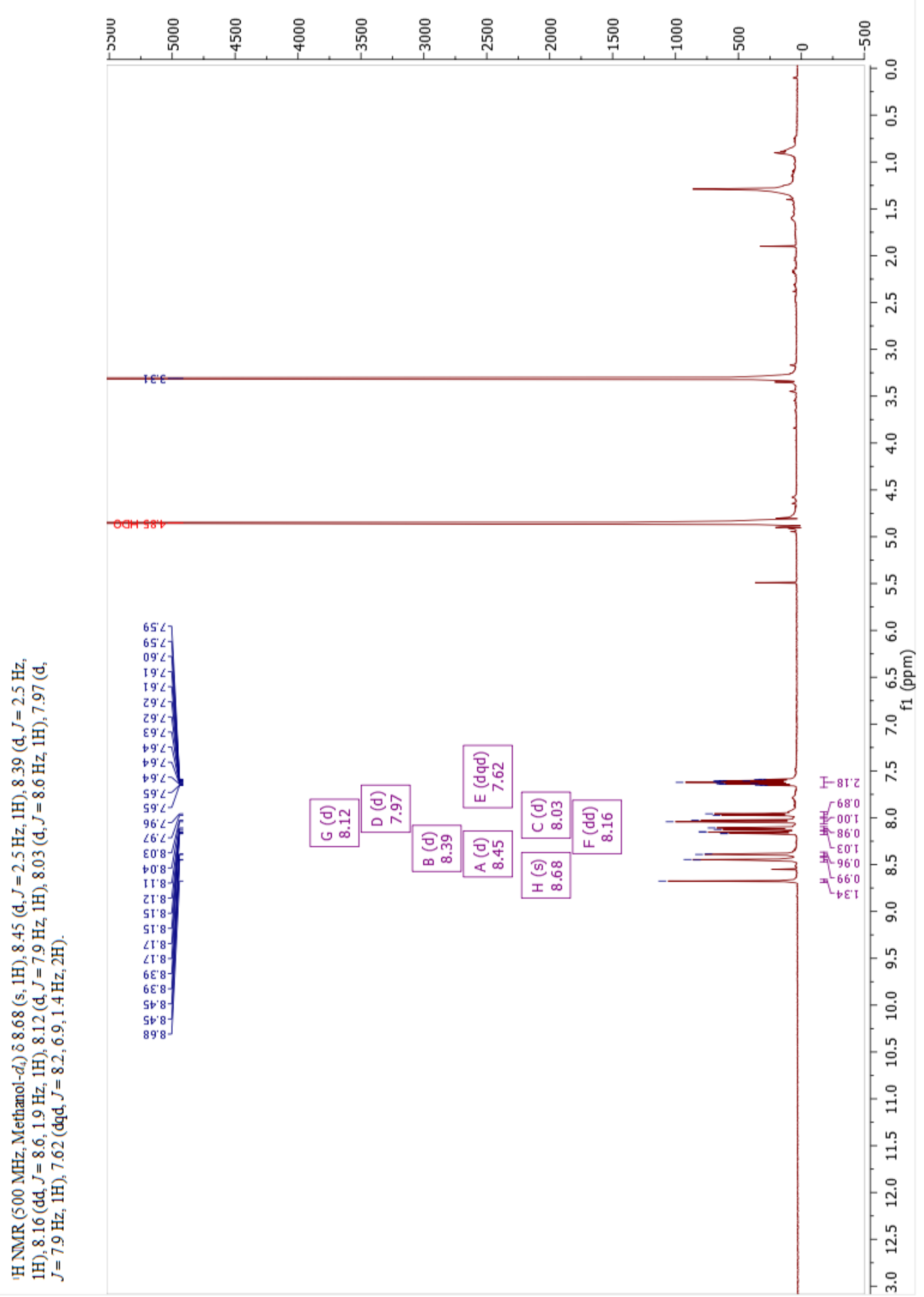




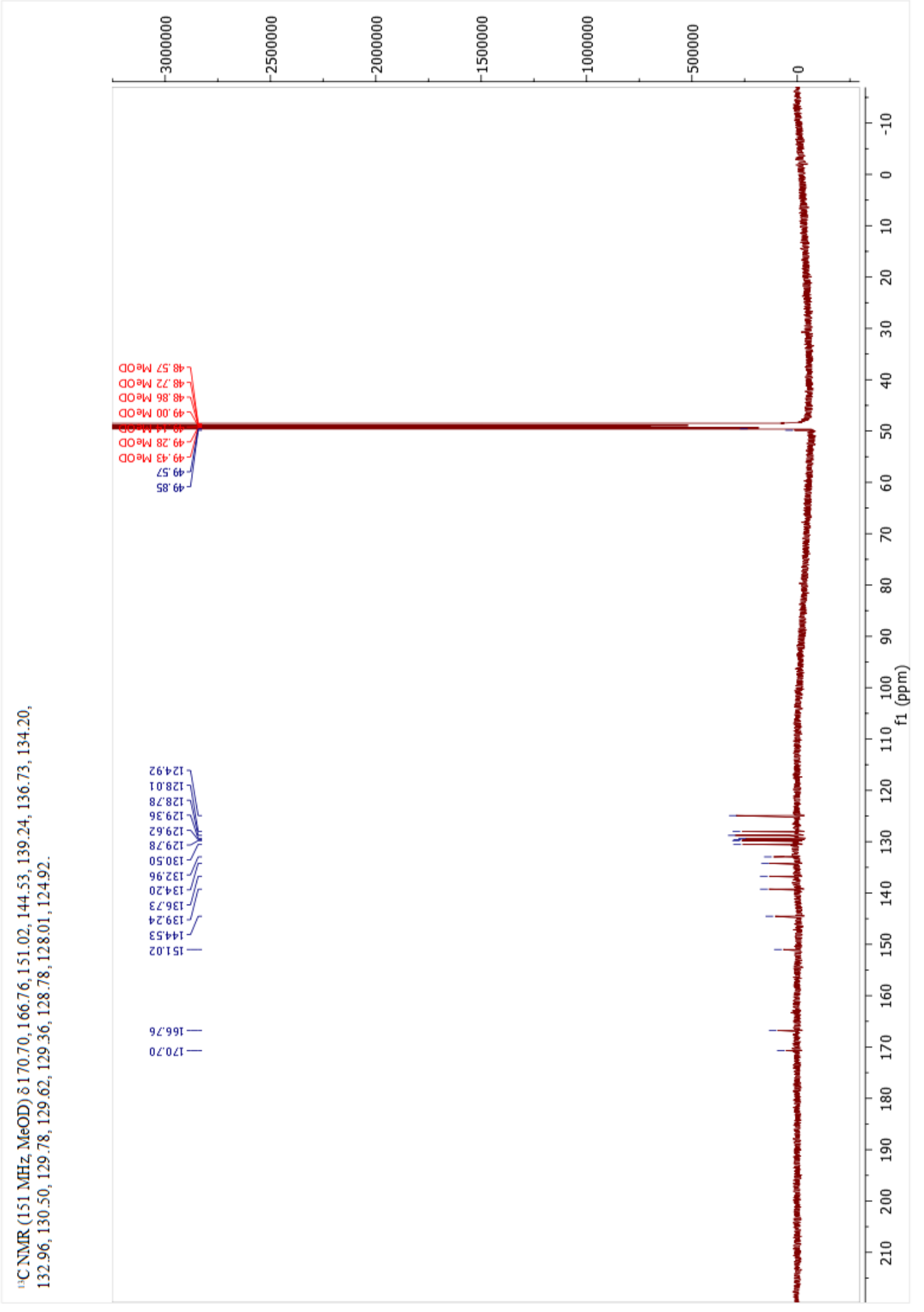




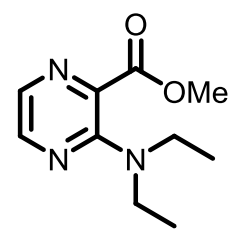

Methyl 3-(diethylamino)pyrazine-2-carboxylate (13-OMe)

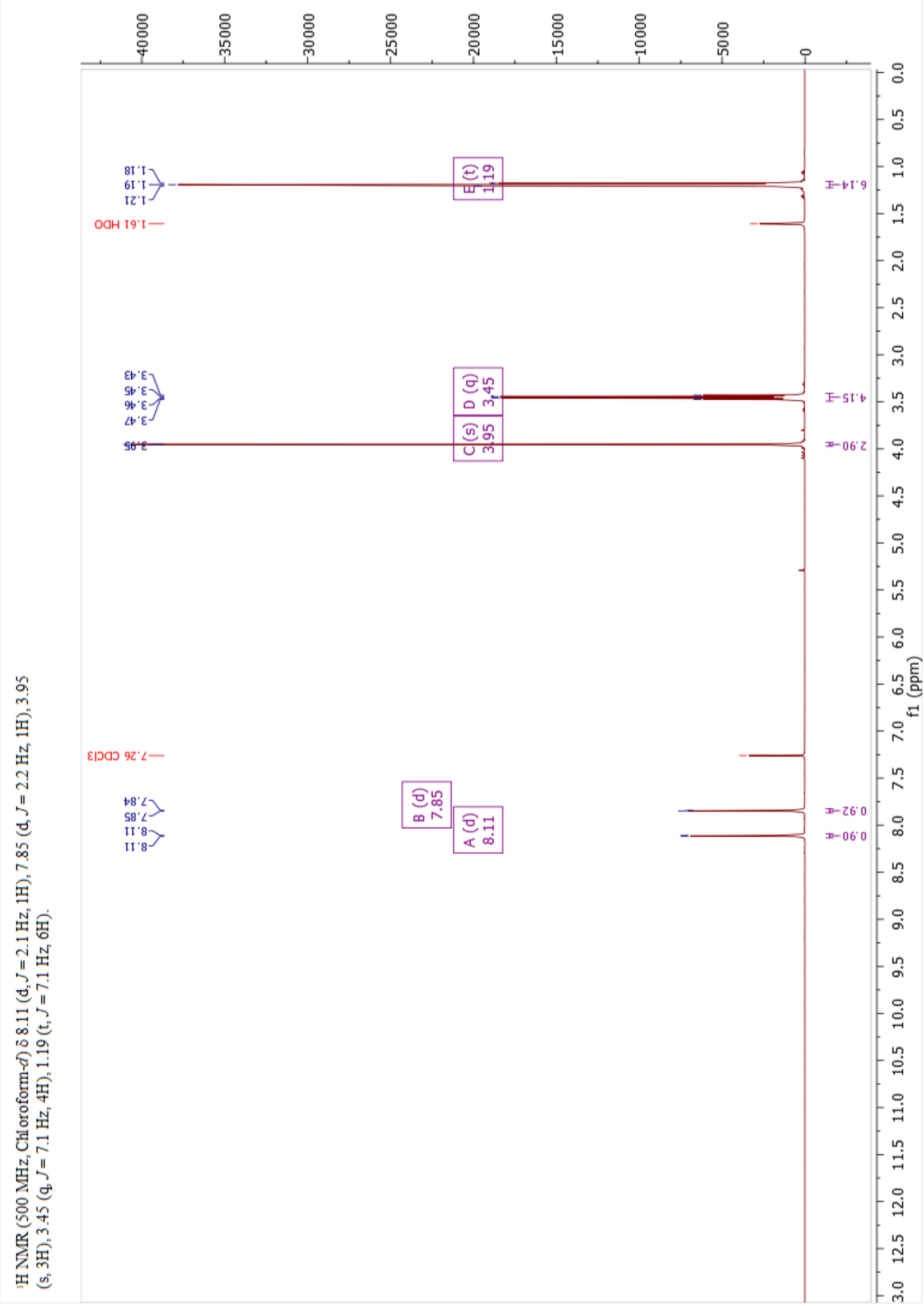




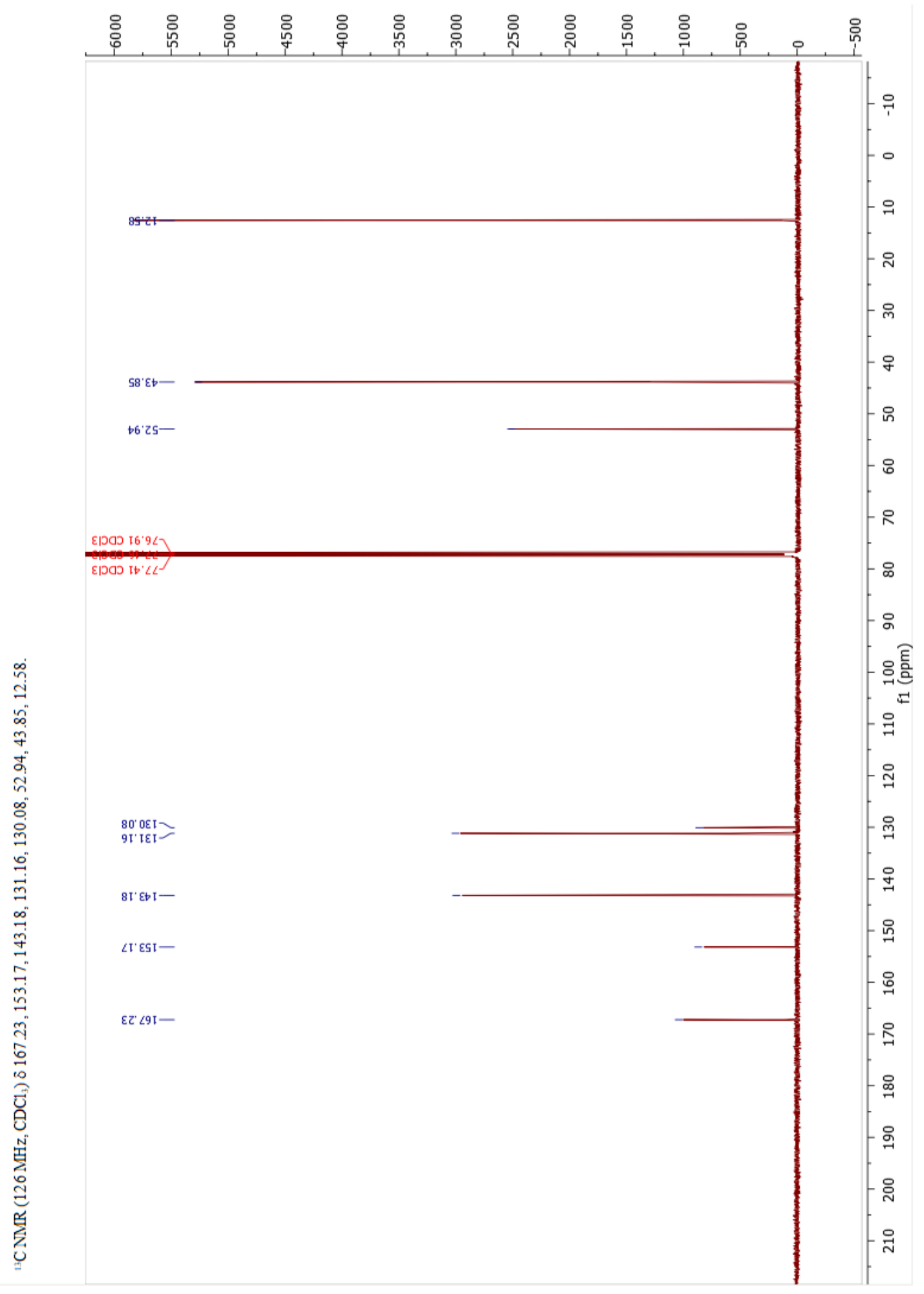




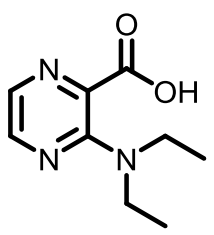

3-(Diethylamino)pyrazine-2-carboxylic acid (13)

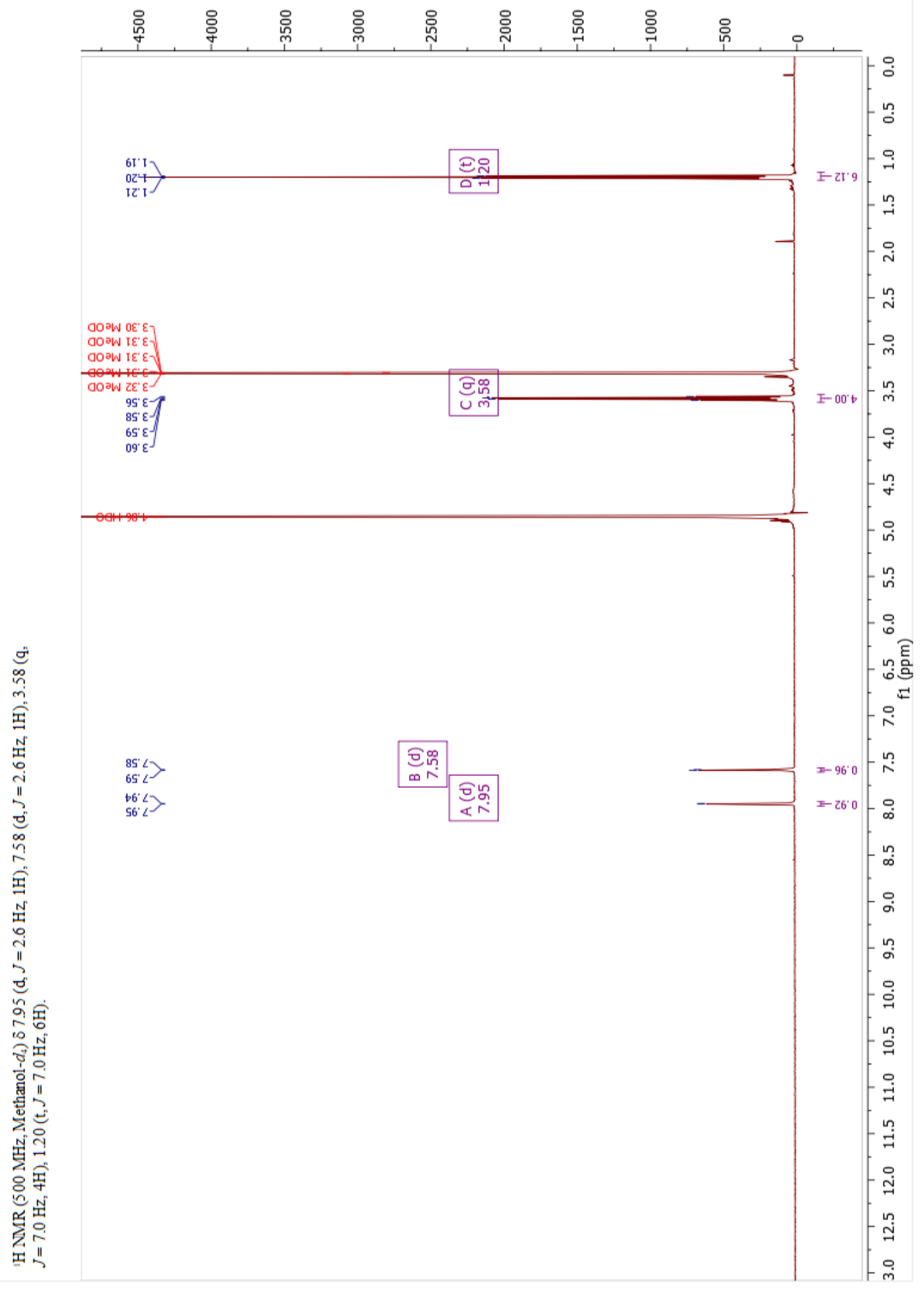




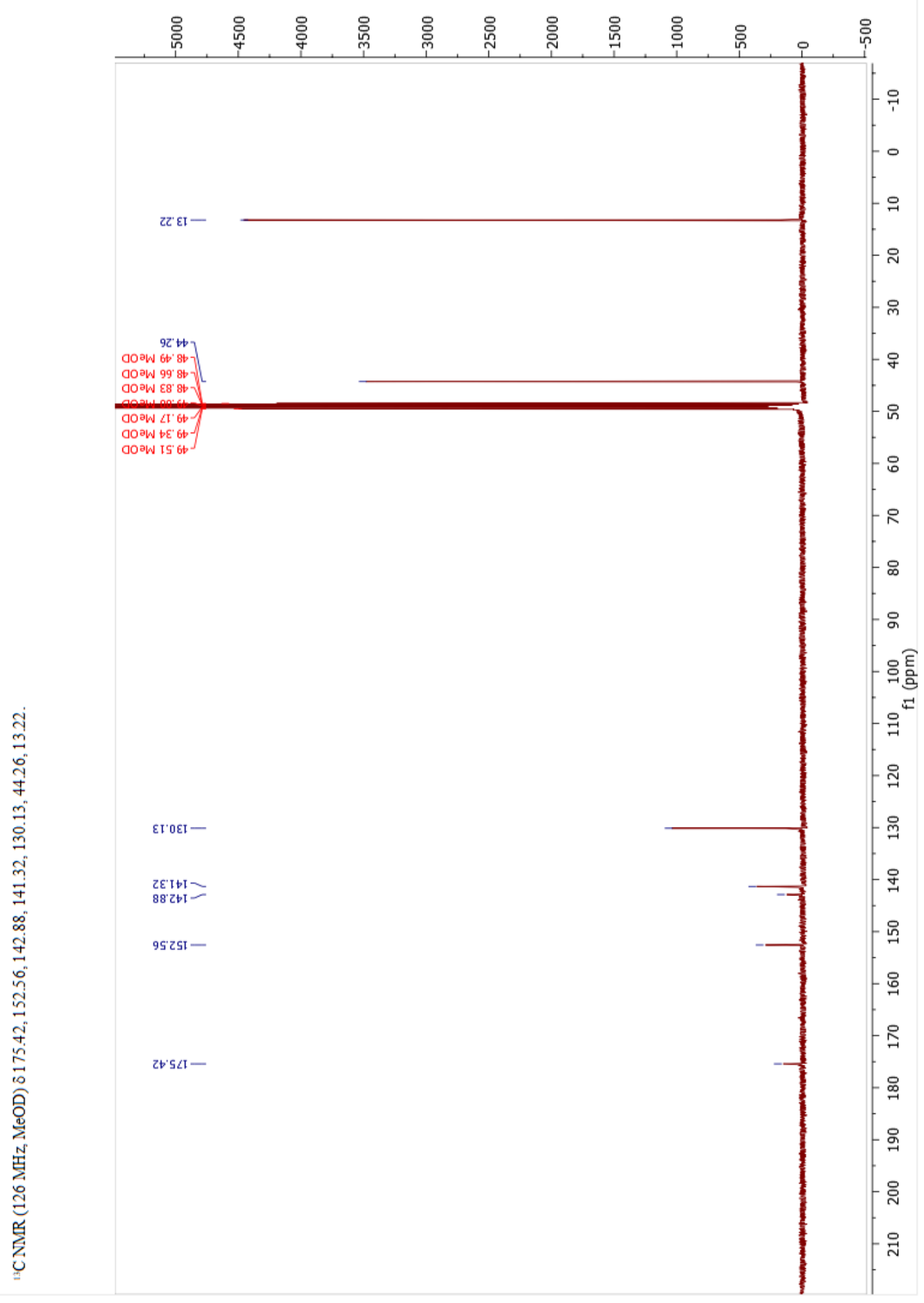


<smiles>COC(=O)c1nccnc1-c1ccccc1</smiles>

Methyl 3-phenylpyrazine-2-carboxylate (14-OMe)

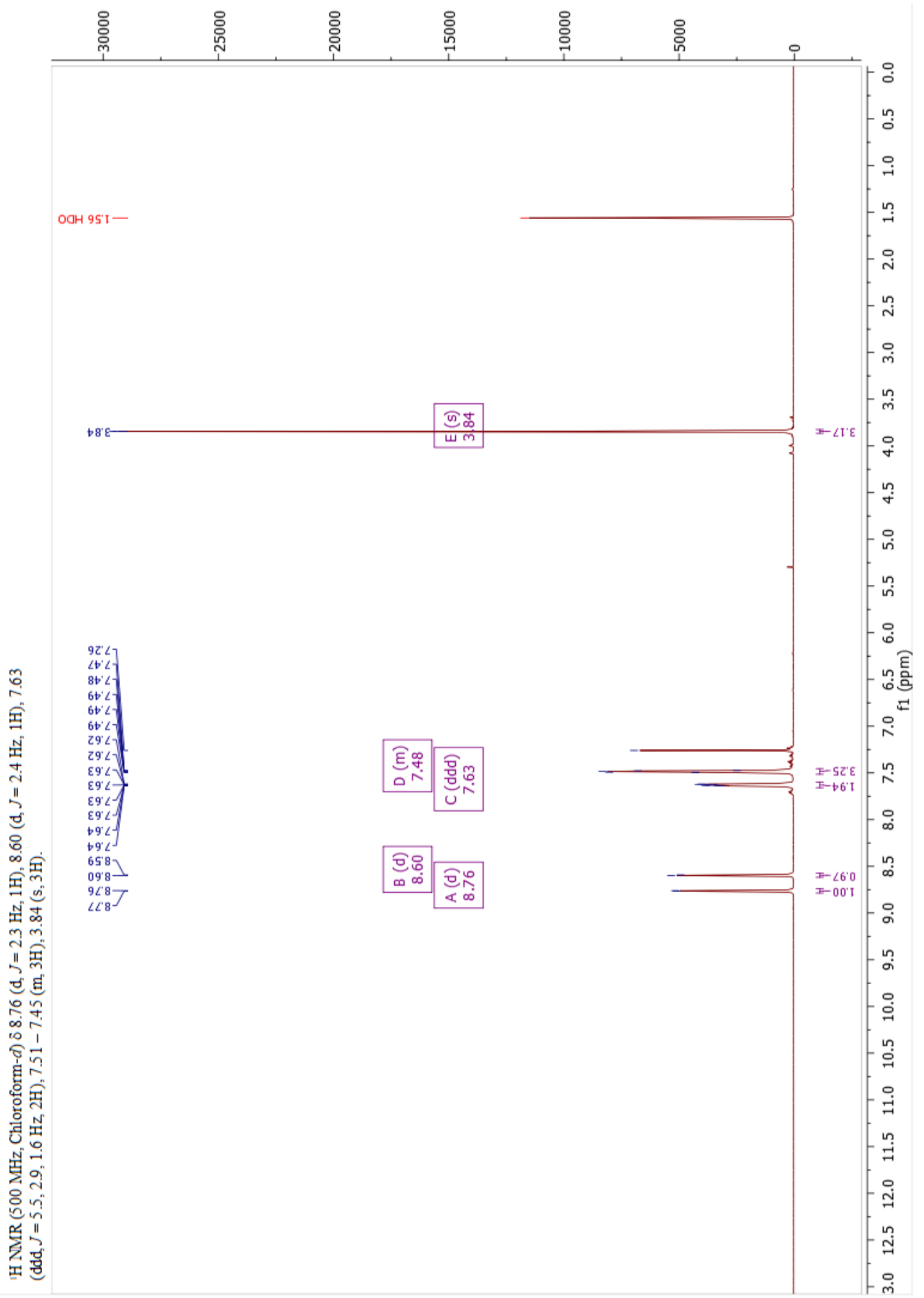


<smiles>O=C(O)c1nccnc1-c1ccccc1</smiles>

3-Phenylpyrazine-2-carboxylic acid (14)

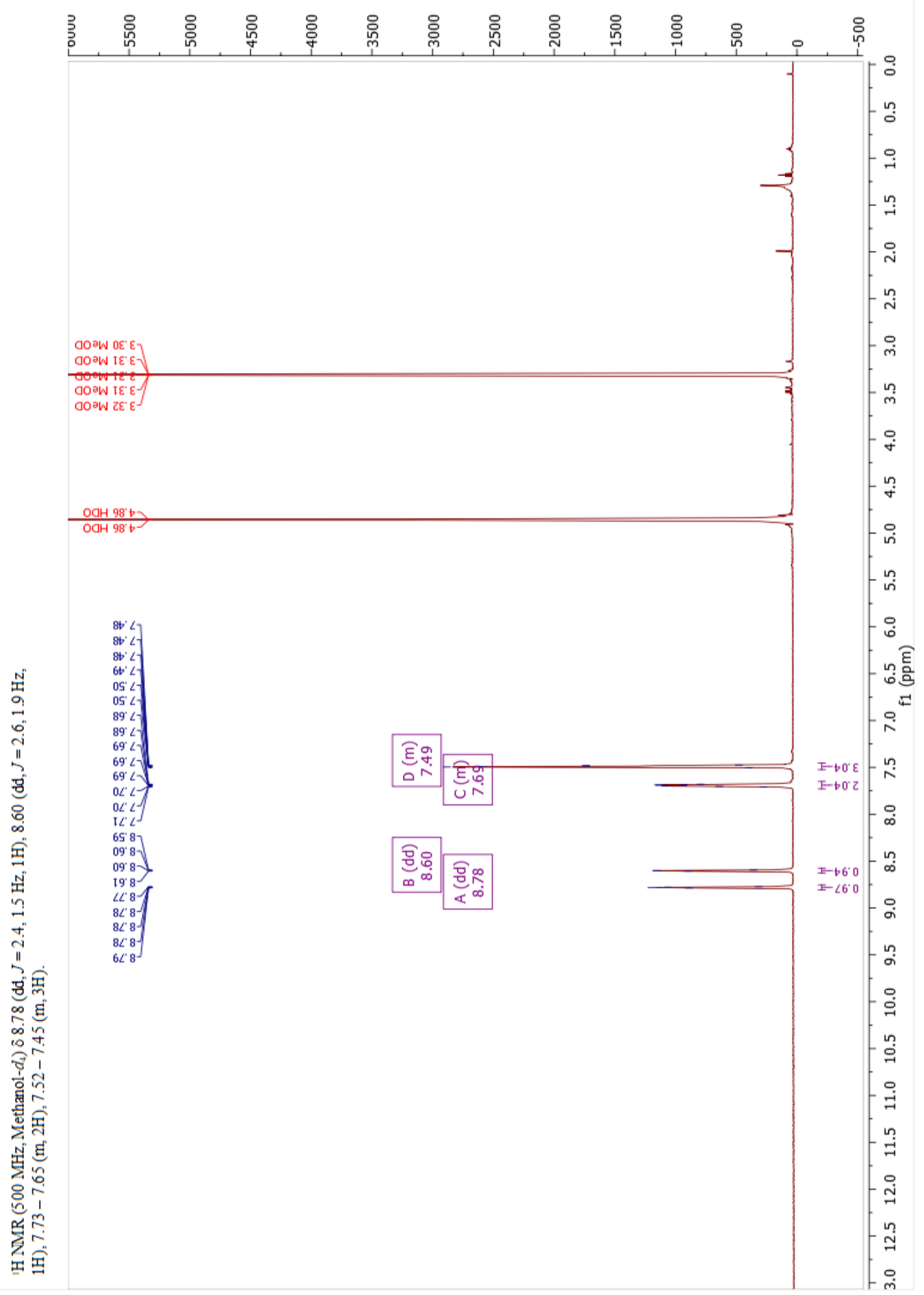


<smiles>COC(=O)c1nccnc1-c1ccc(C)cc1</smiles>

Methyl 3-(4-(methylthio)phenyl)pyrazine-2-carboxylate (15-OMe)

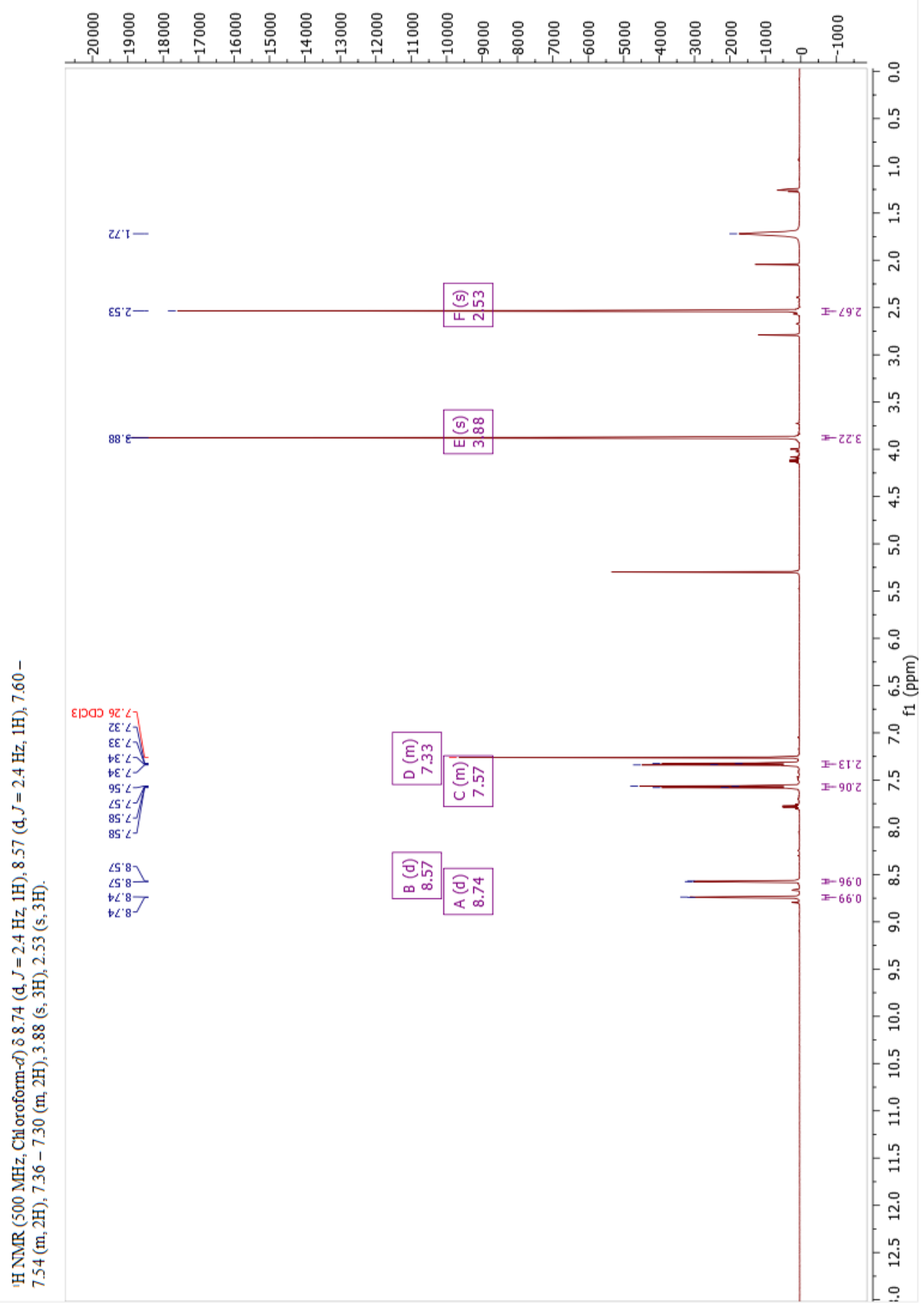




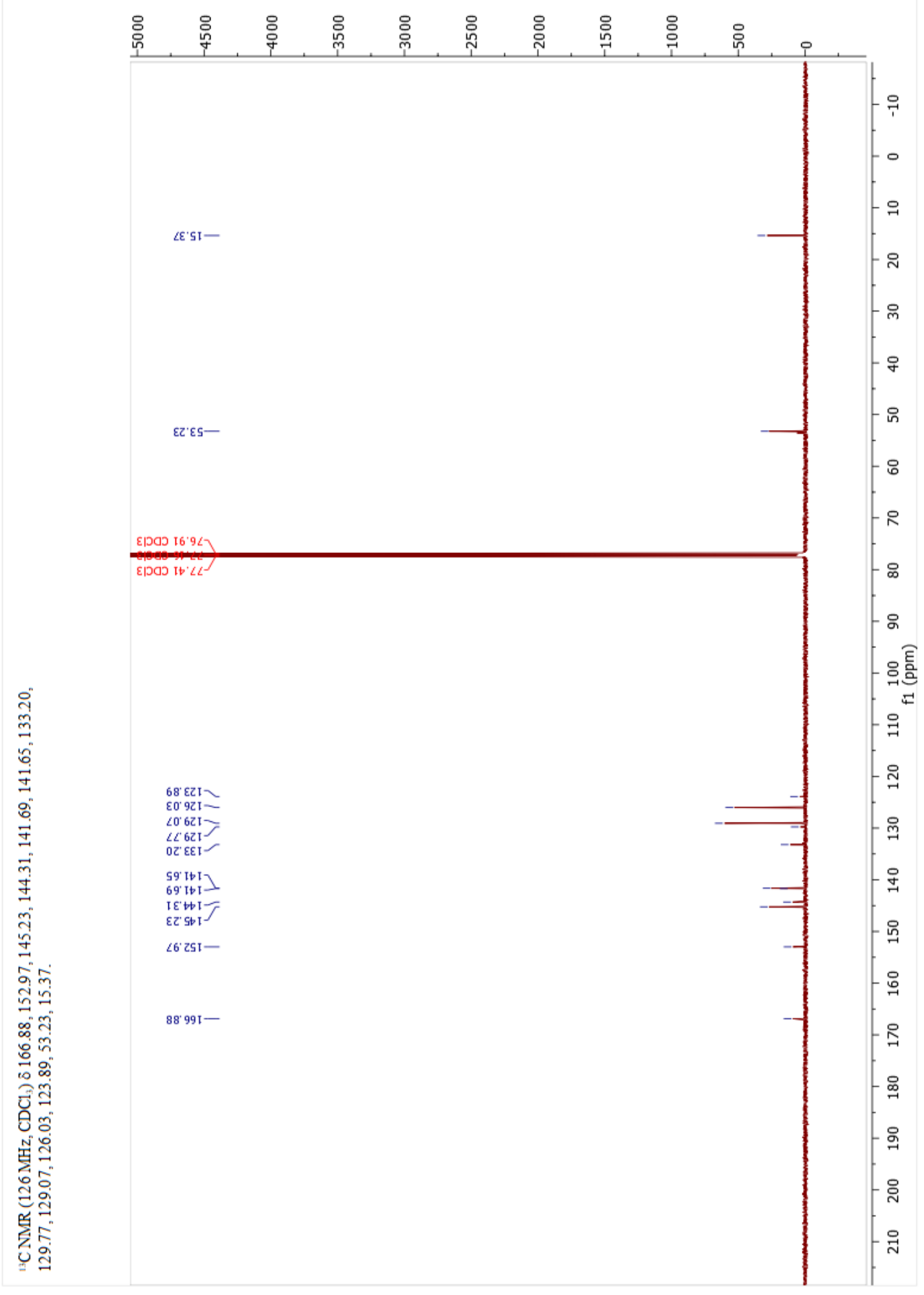


<smiles>CSc1ccc(-c2nccnc2C(=O)O)cc1</smiles>

3-(4-(Methylthio)phenyl)pyrazine-2-carboxylic acid (15)

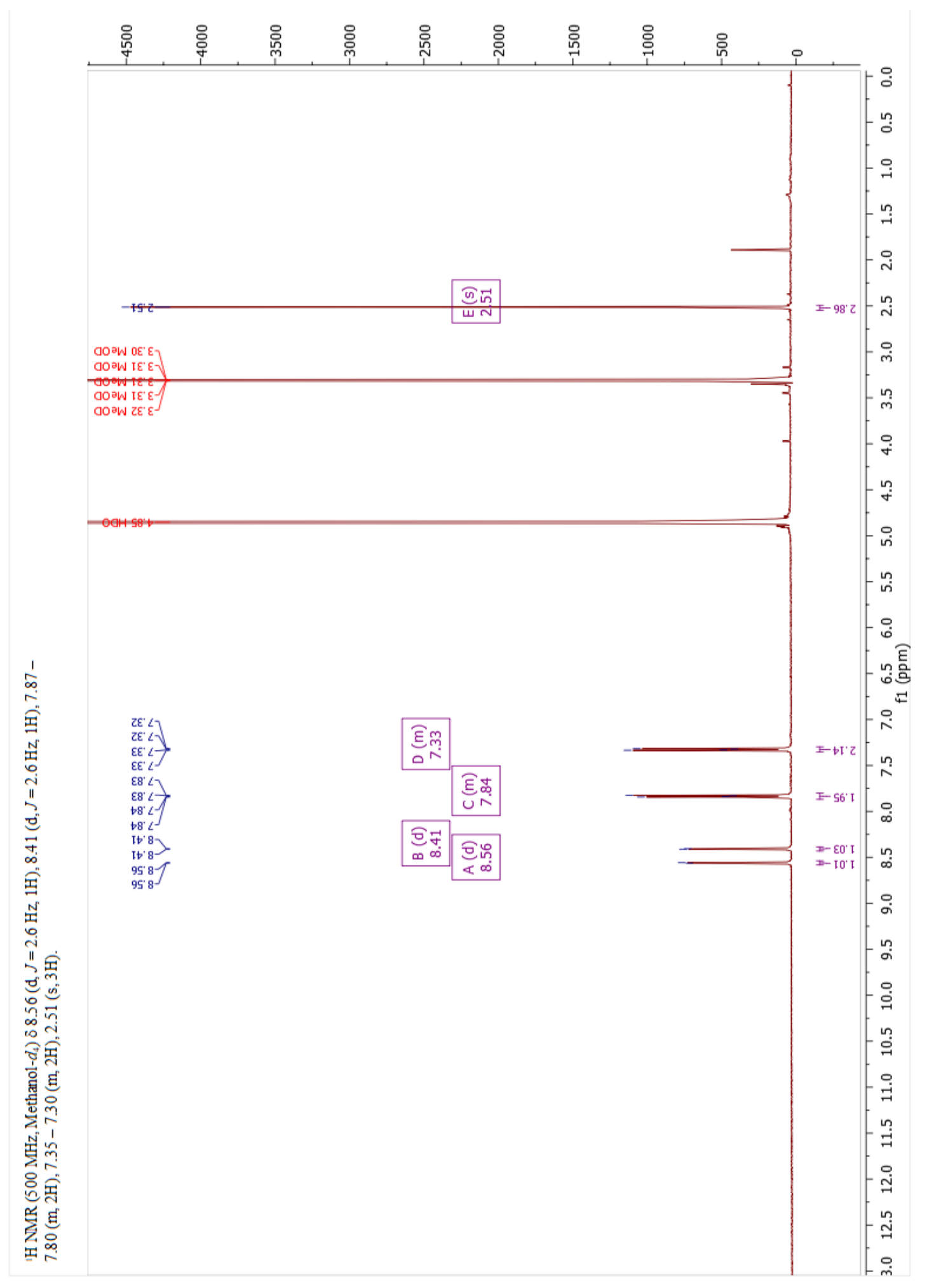

S77 


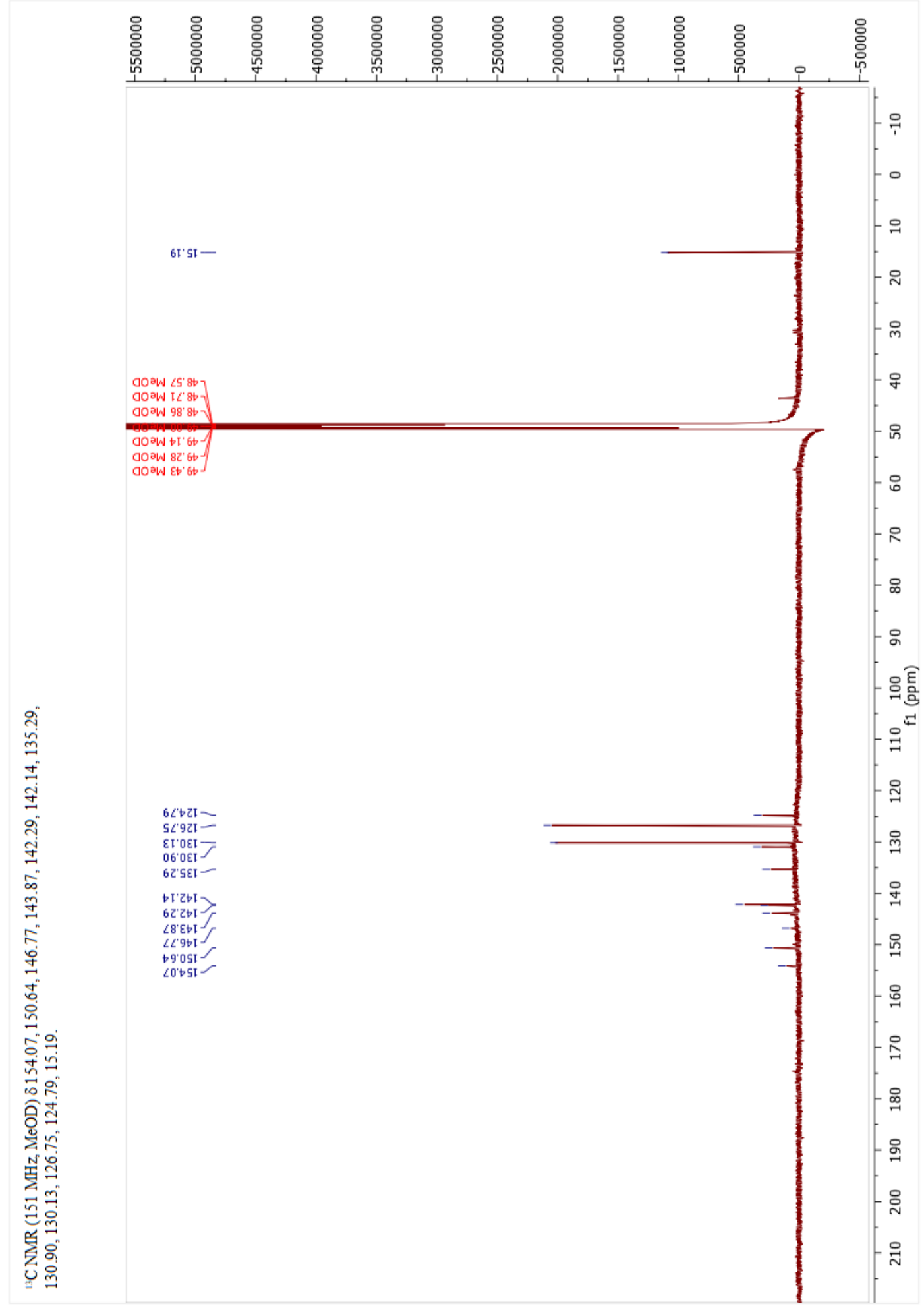


<smiles>COC(=O)c1nccnc1-c1ccccc1C</smiles>

Methyl 3-(o-tolyl)pyrazine-2-carboxylate (16-OMe)

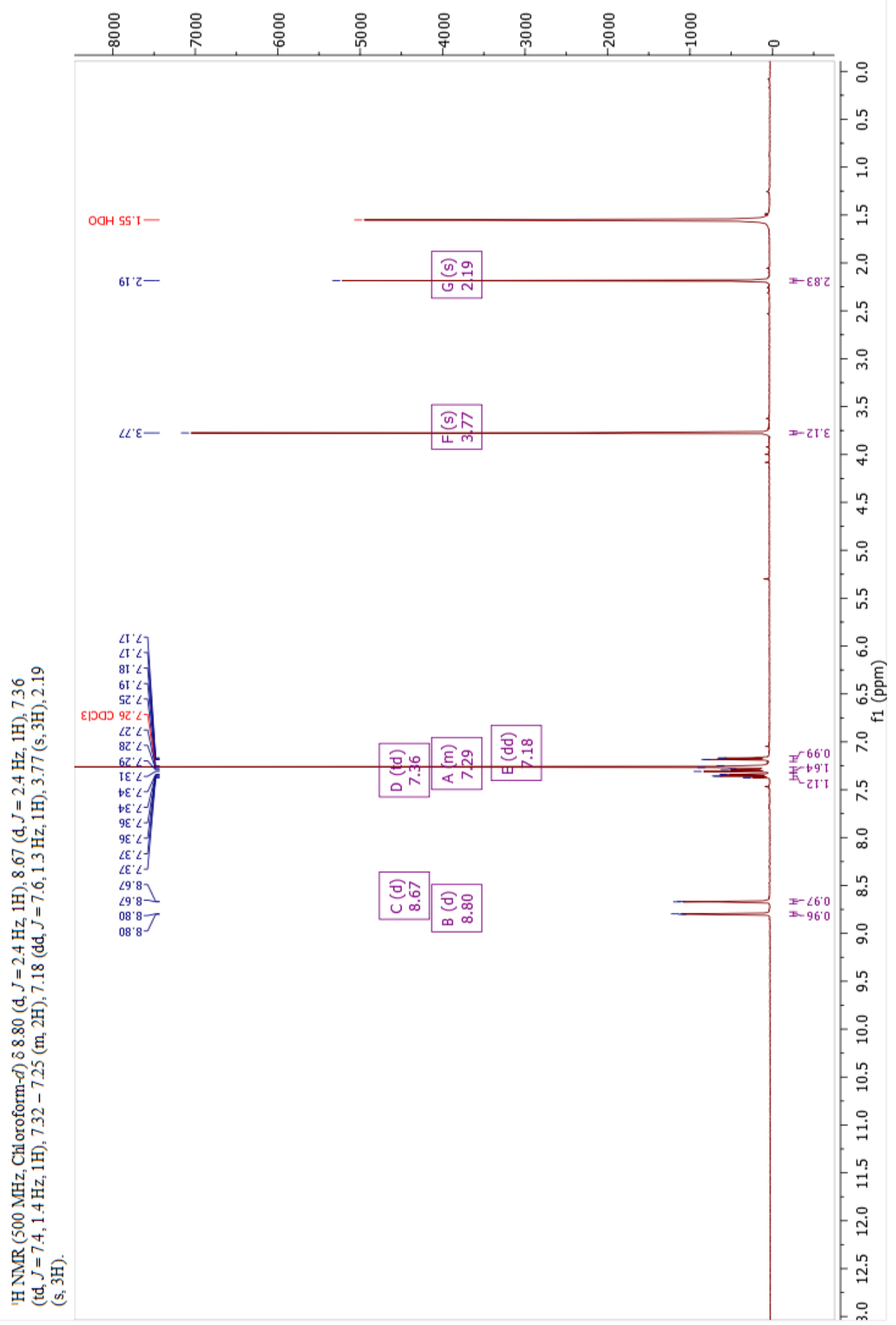




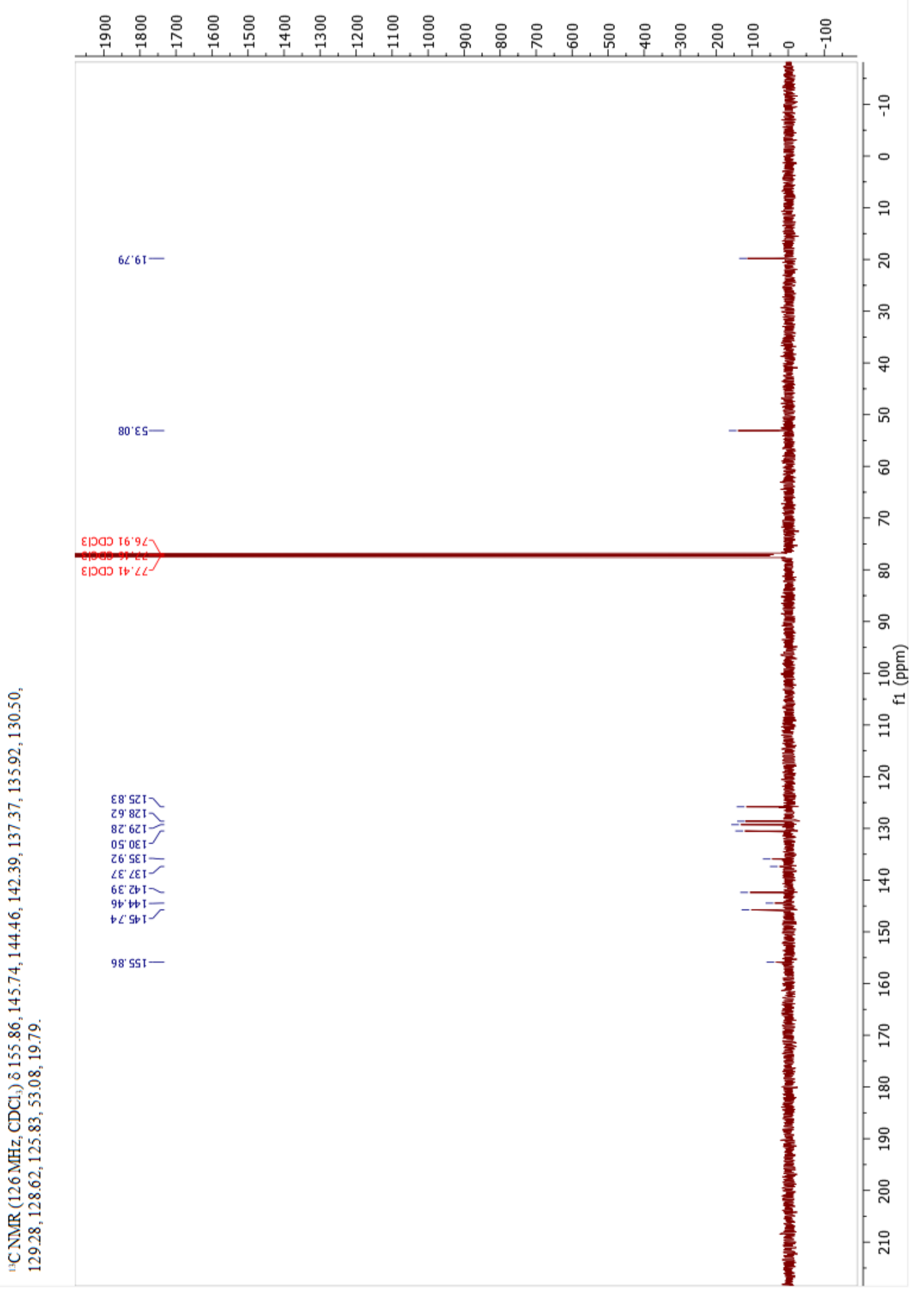




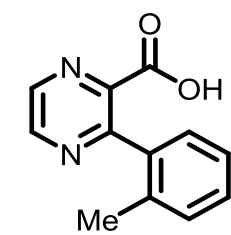

3-(o-Tolyl)pyrazine-2-carboxylic acid (16)

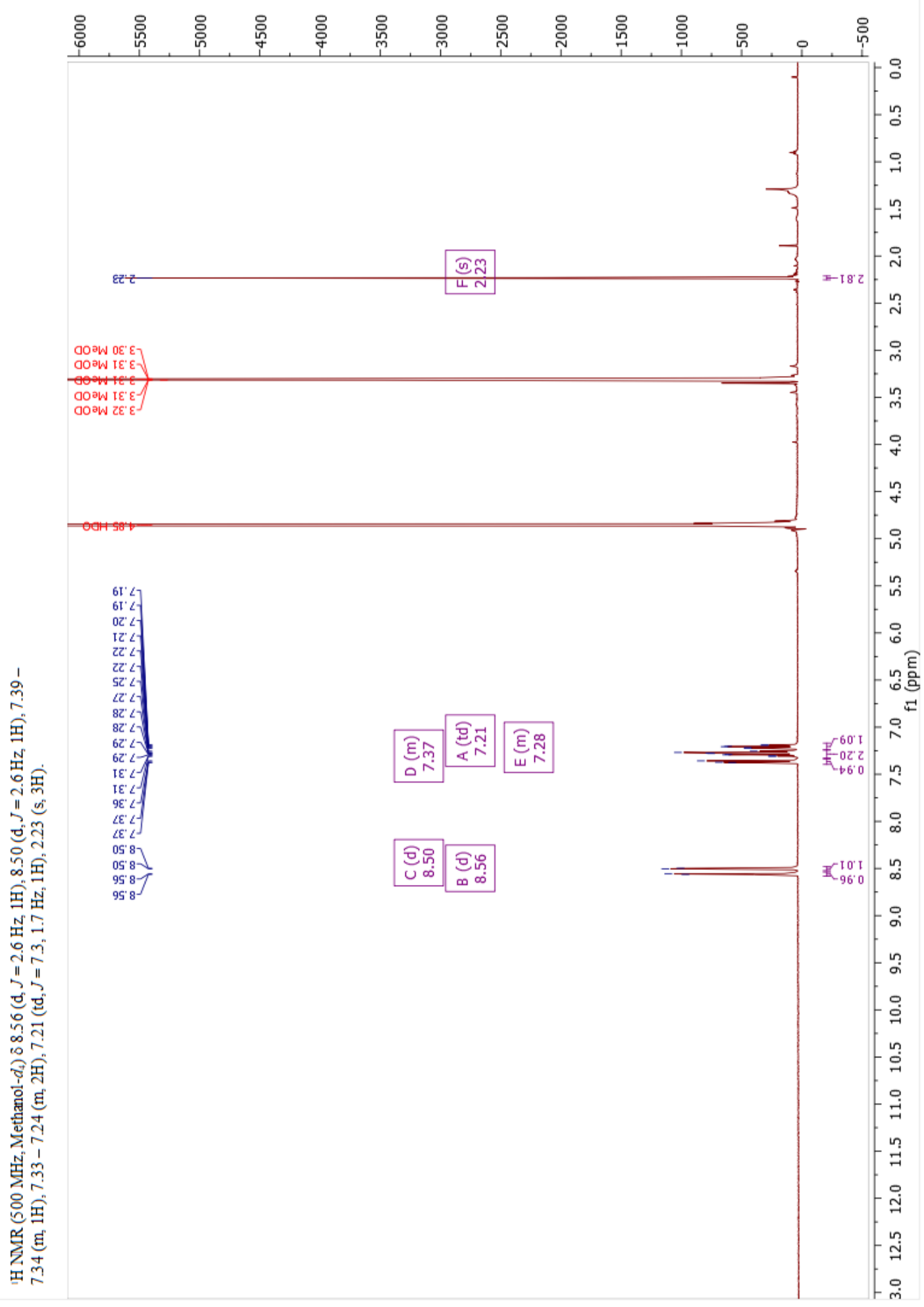




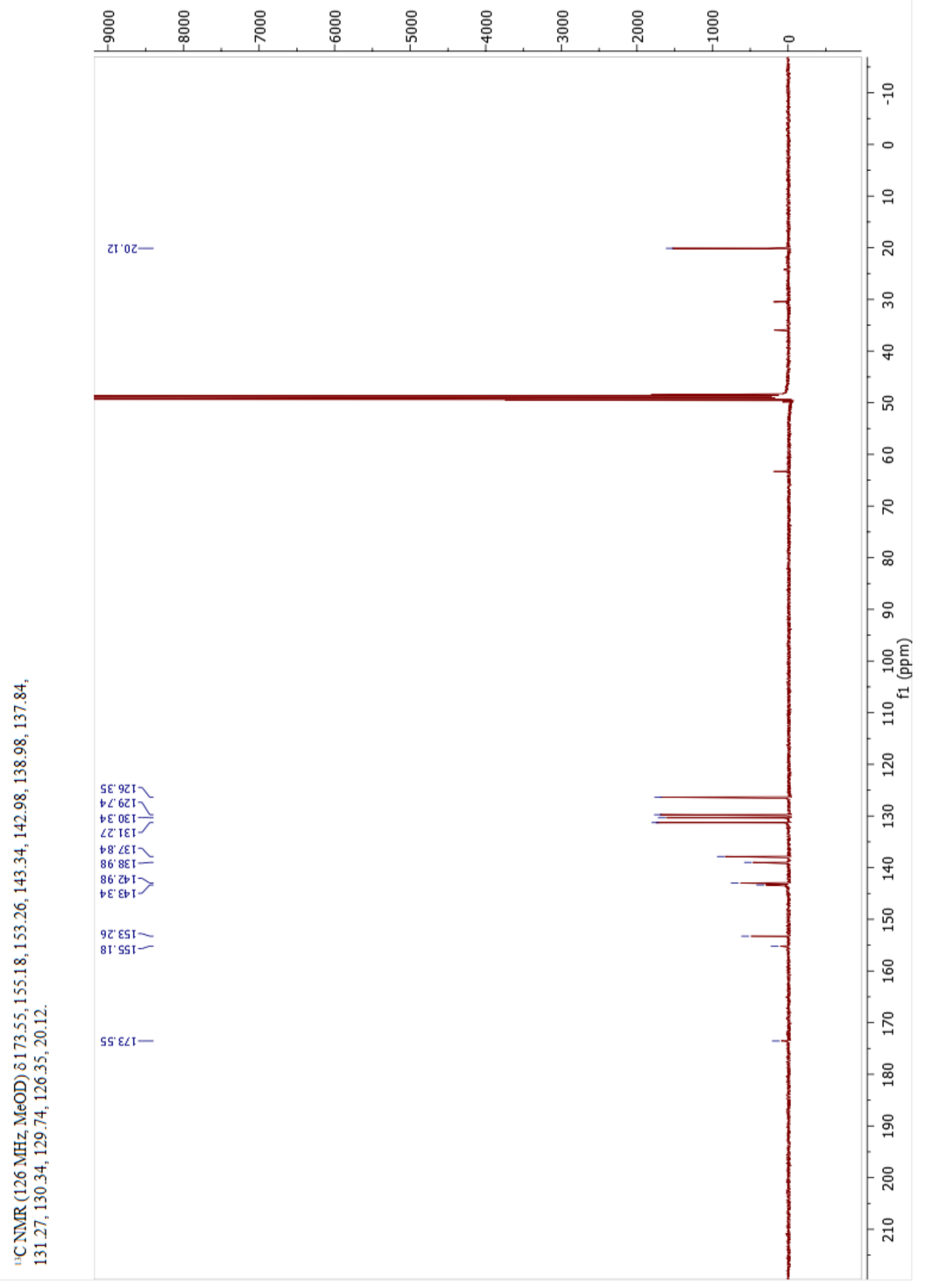




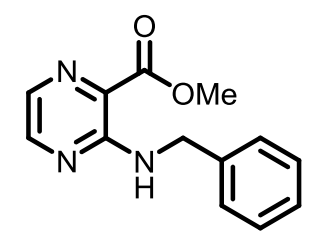

Methyl 3-(benzylamino)pyrazine-2-carboxylate (17-OMe)

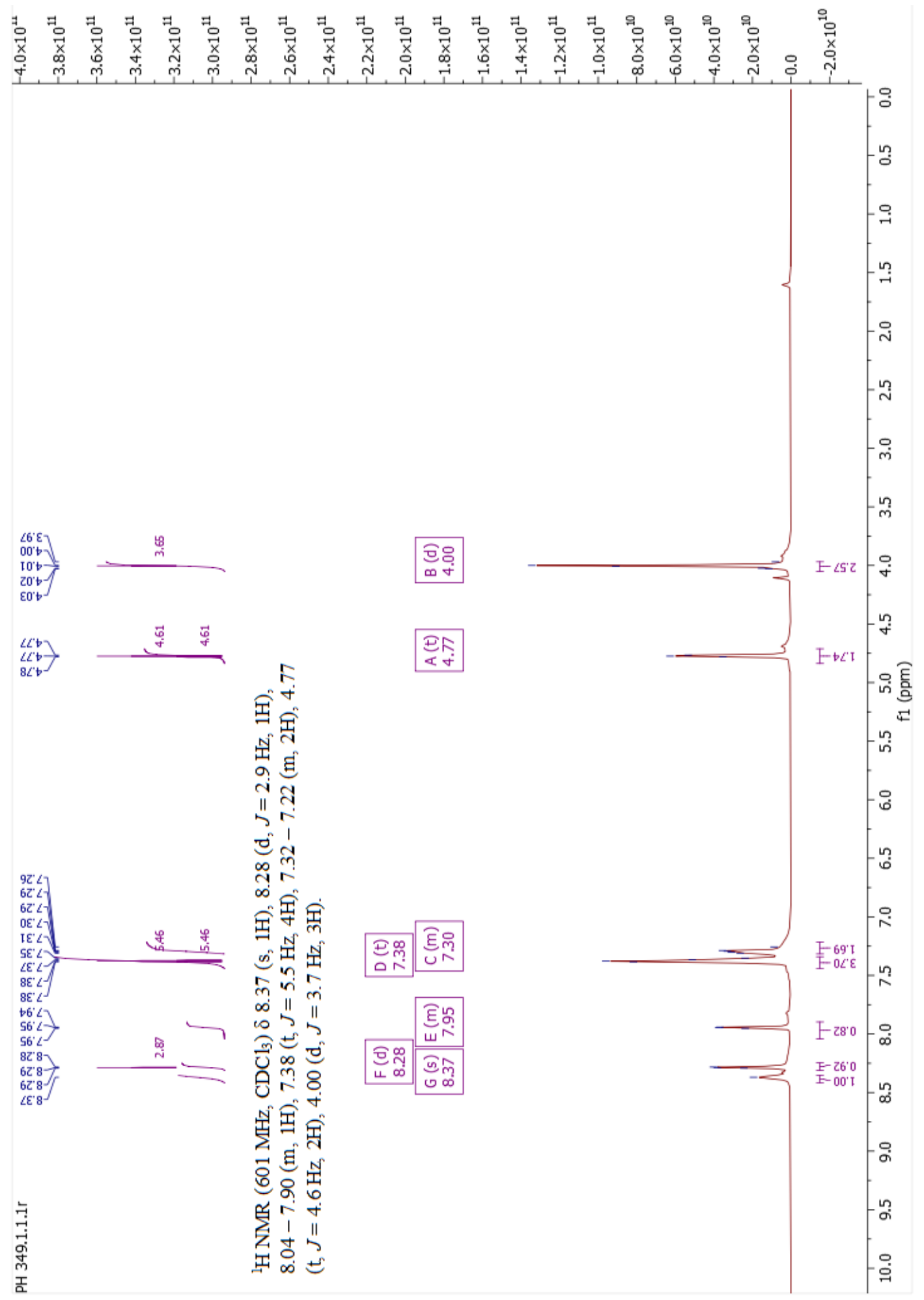




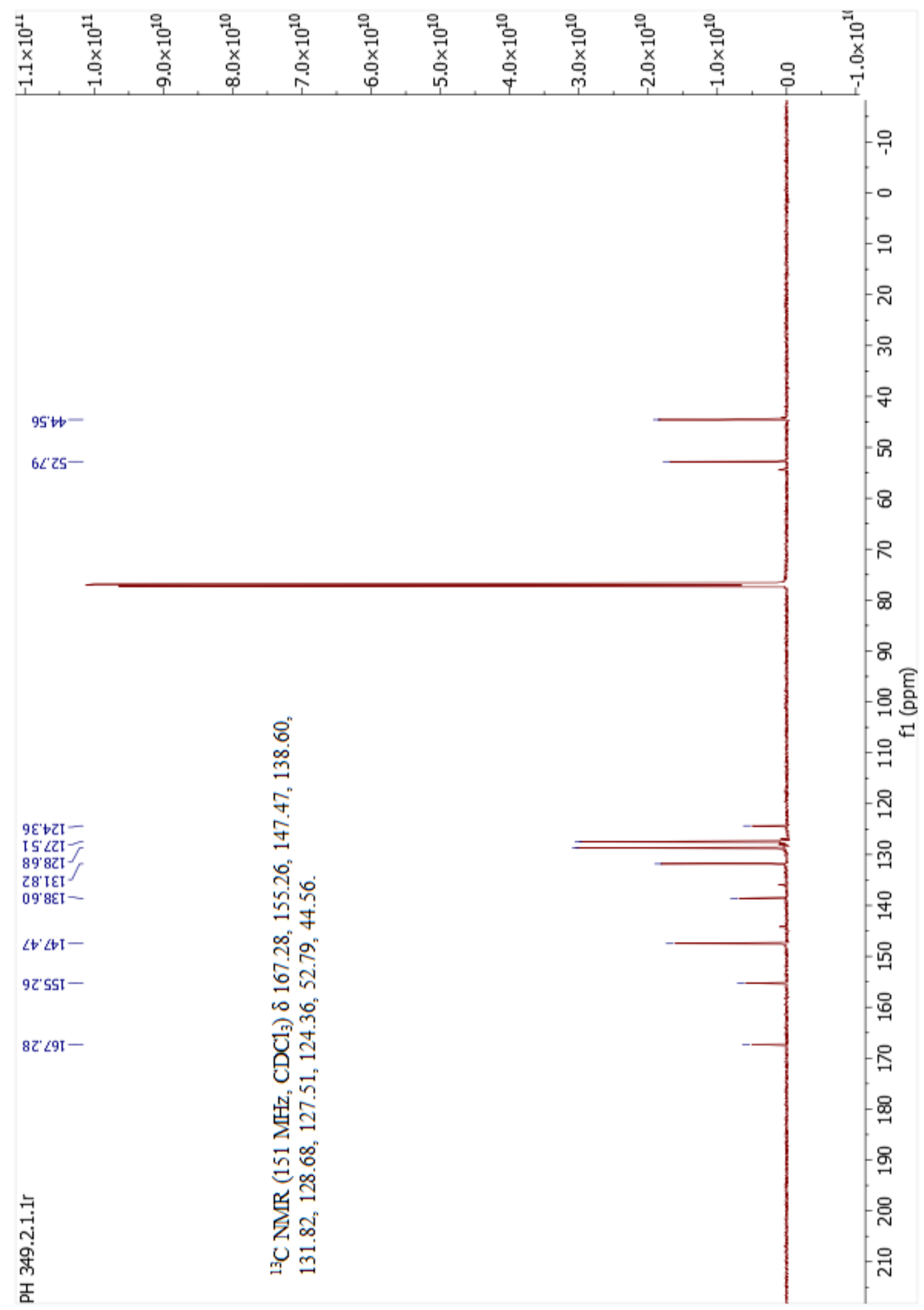


<smiles>O=C(O)c1nccnc1NCc1ccccc1</smiles>

3-(Benzylamino)pyrazine-2-carboxylic acid (17)

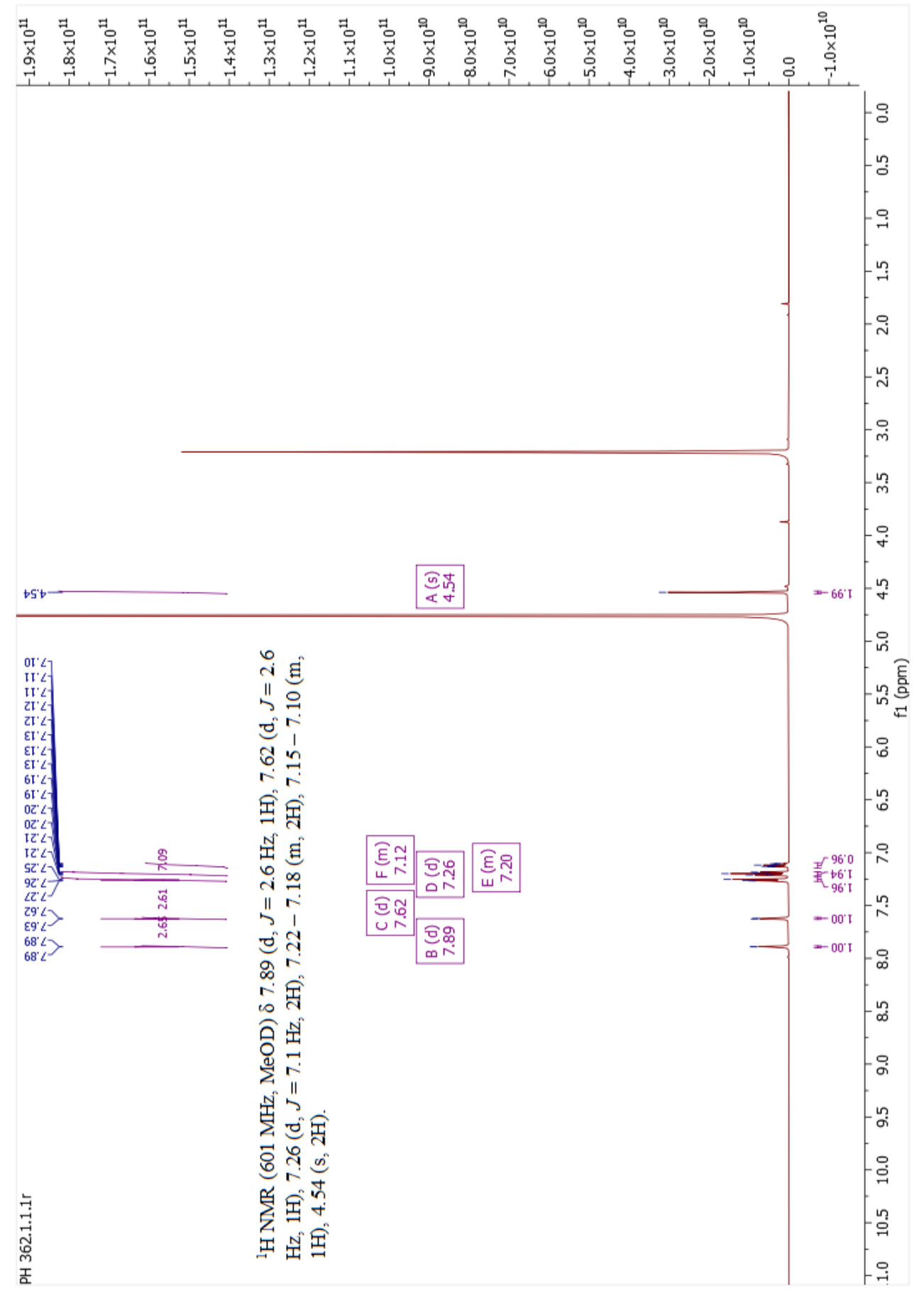




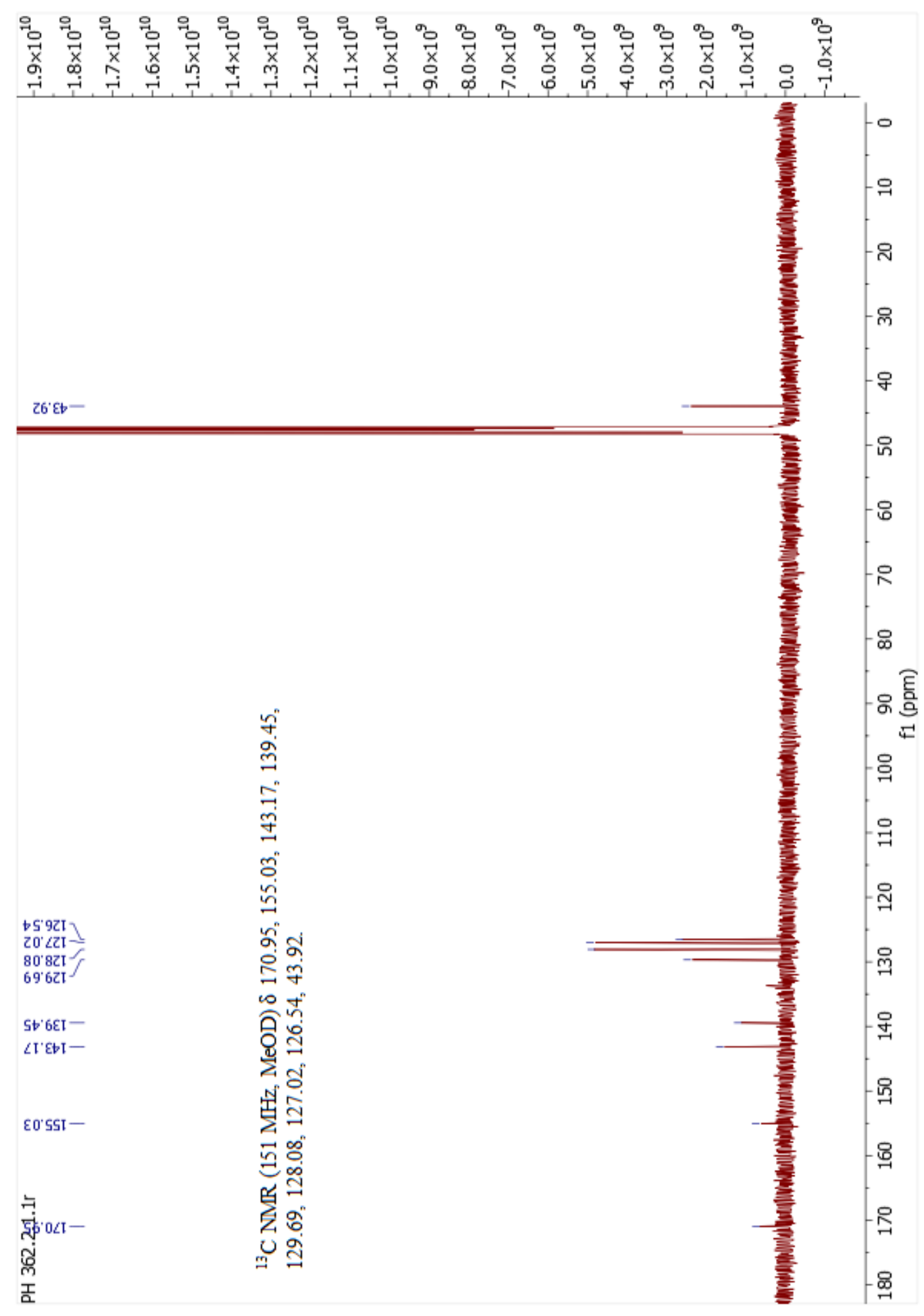




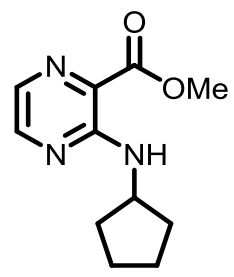

Methyl 3-(cyclopentylamino)pyrazine-2-carboxylate (18-OMe)

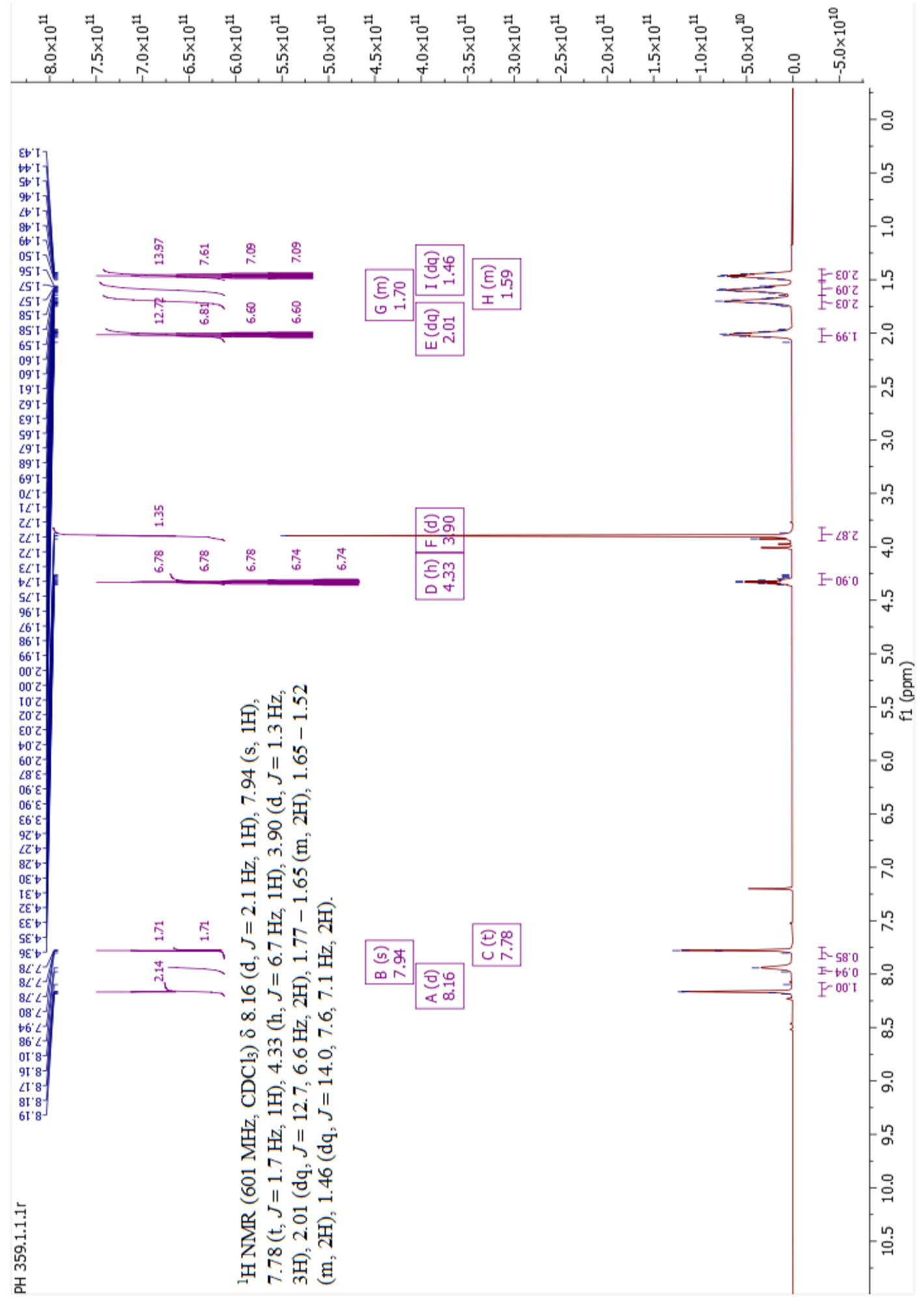




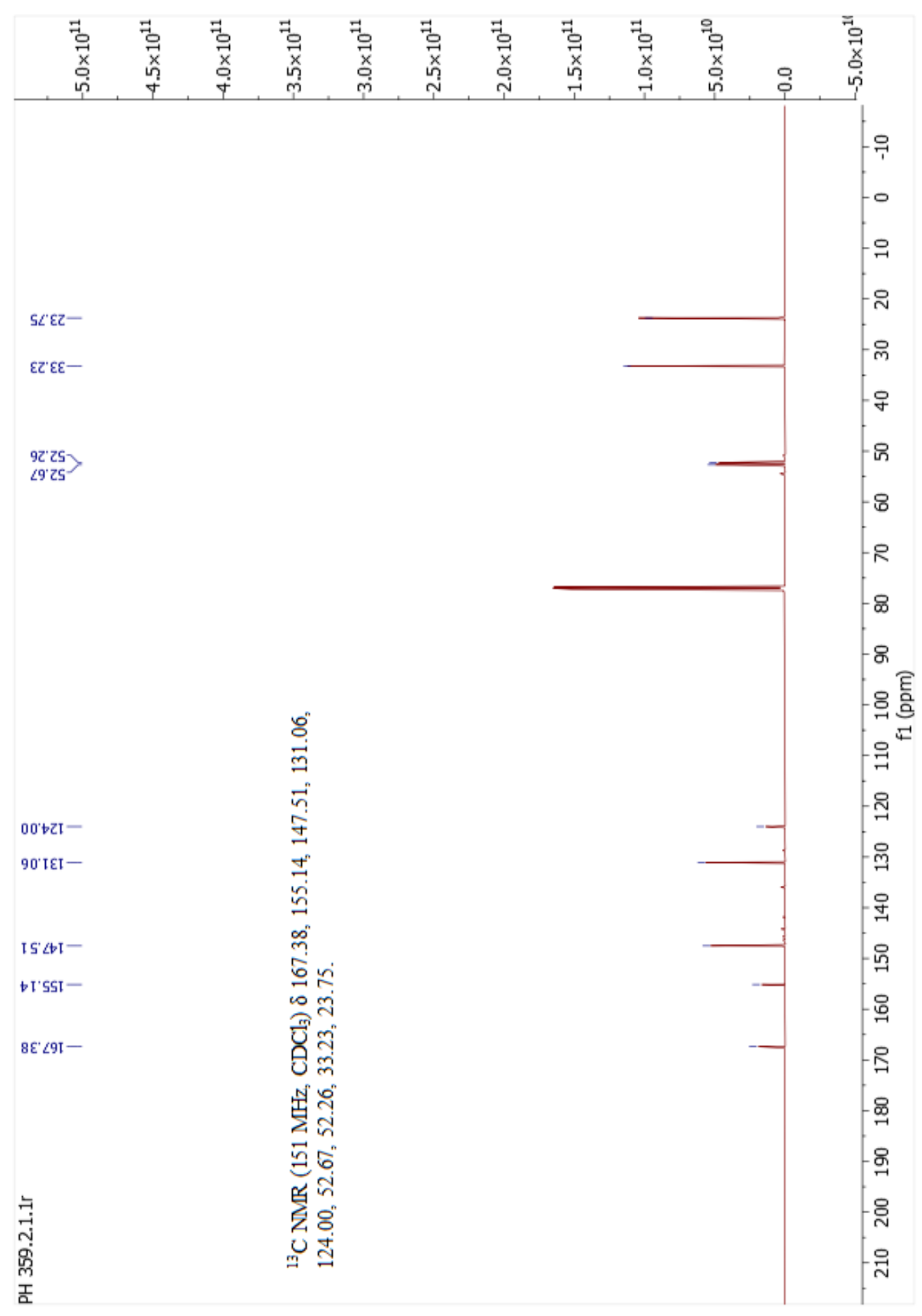




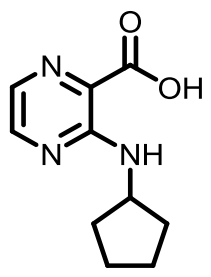

3-(Cyclopentylamino)pyrazine-2-carboxylic acid (18)

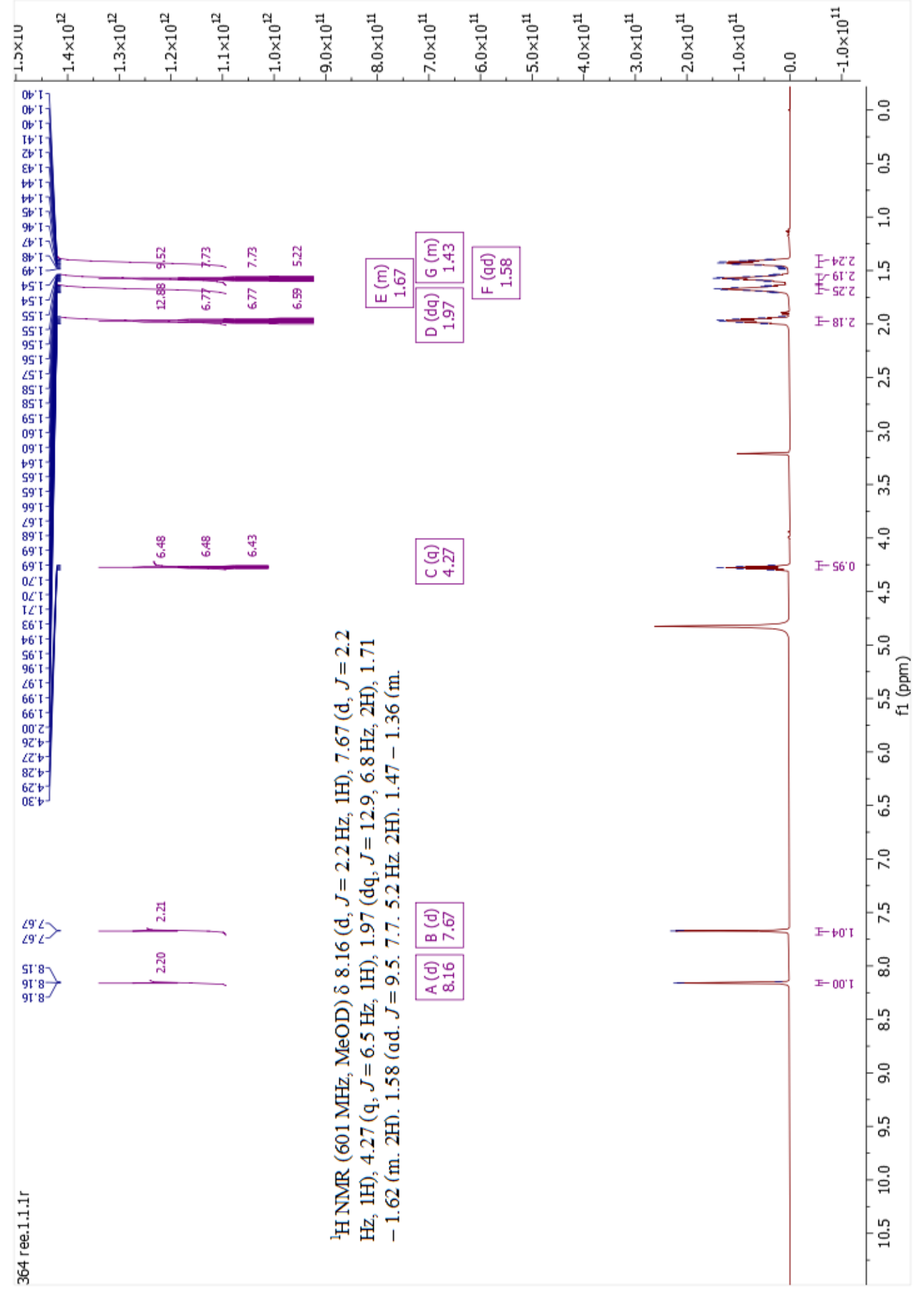




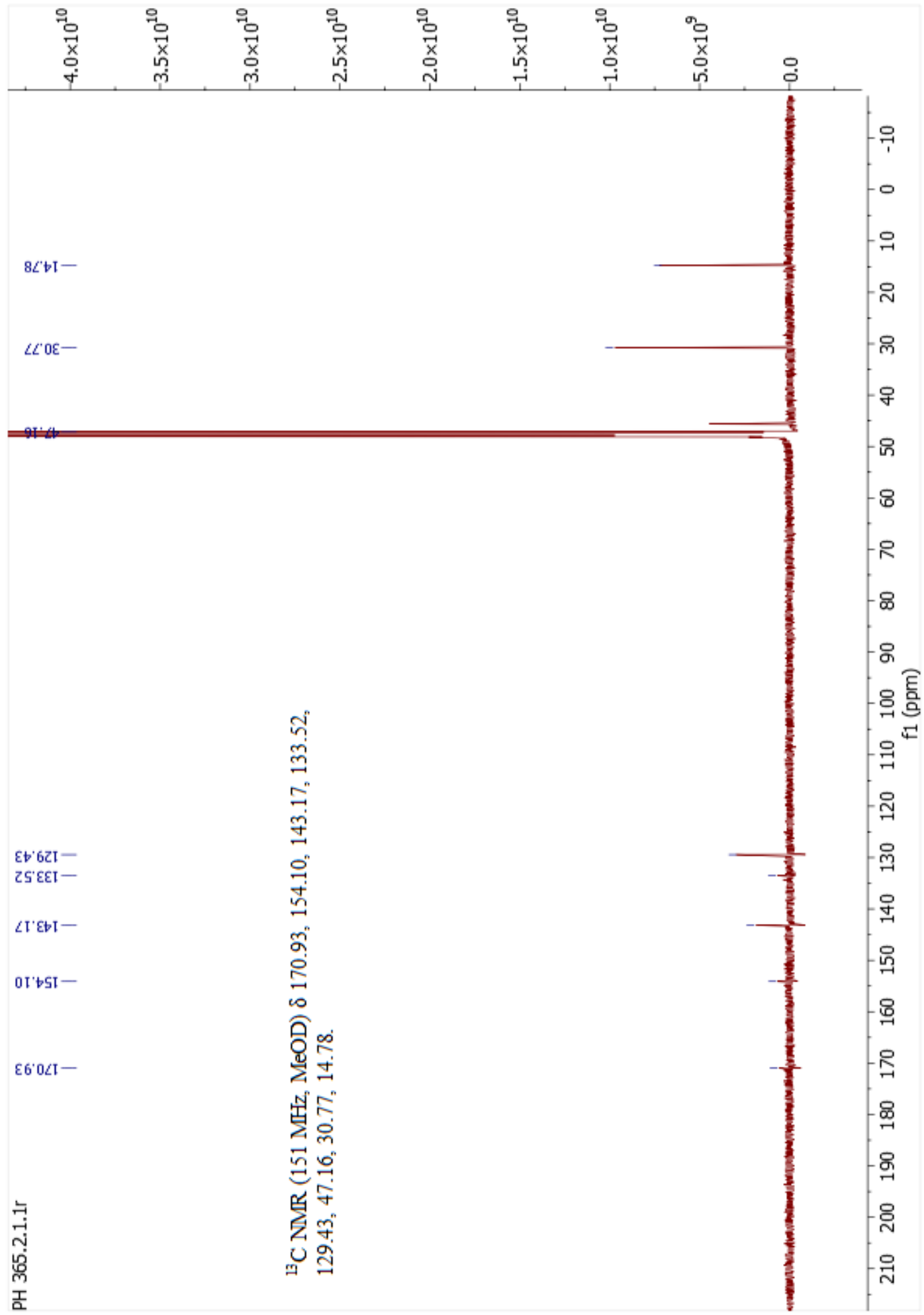

S90 


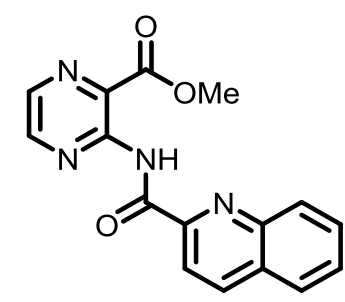

Methyl 3-(quinoline-2-carboxamido)pyrazine-2-carboxylate (19-OMe)

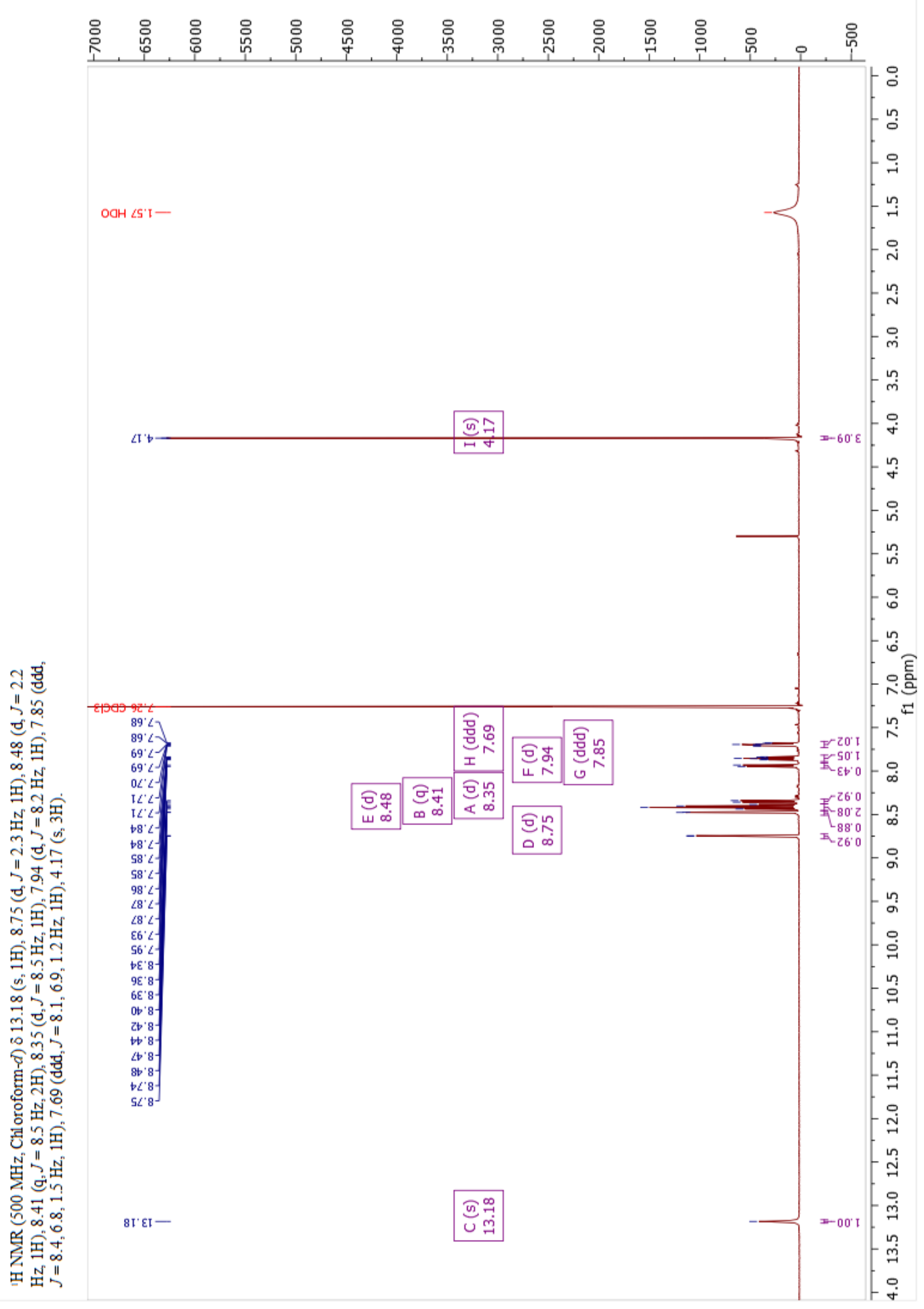




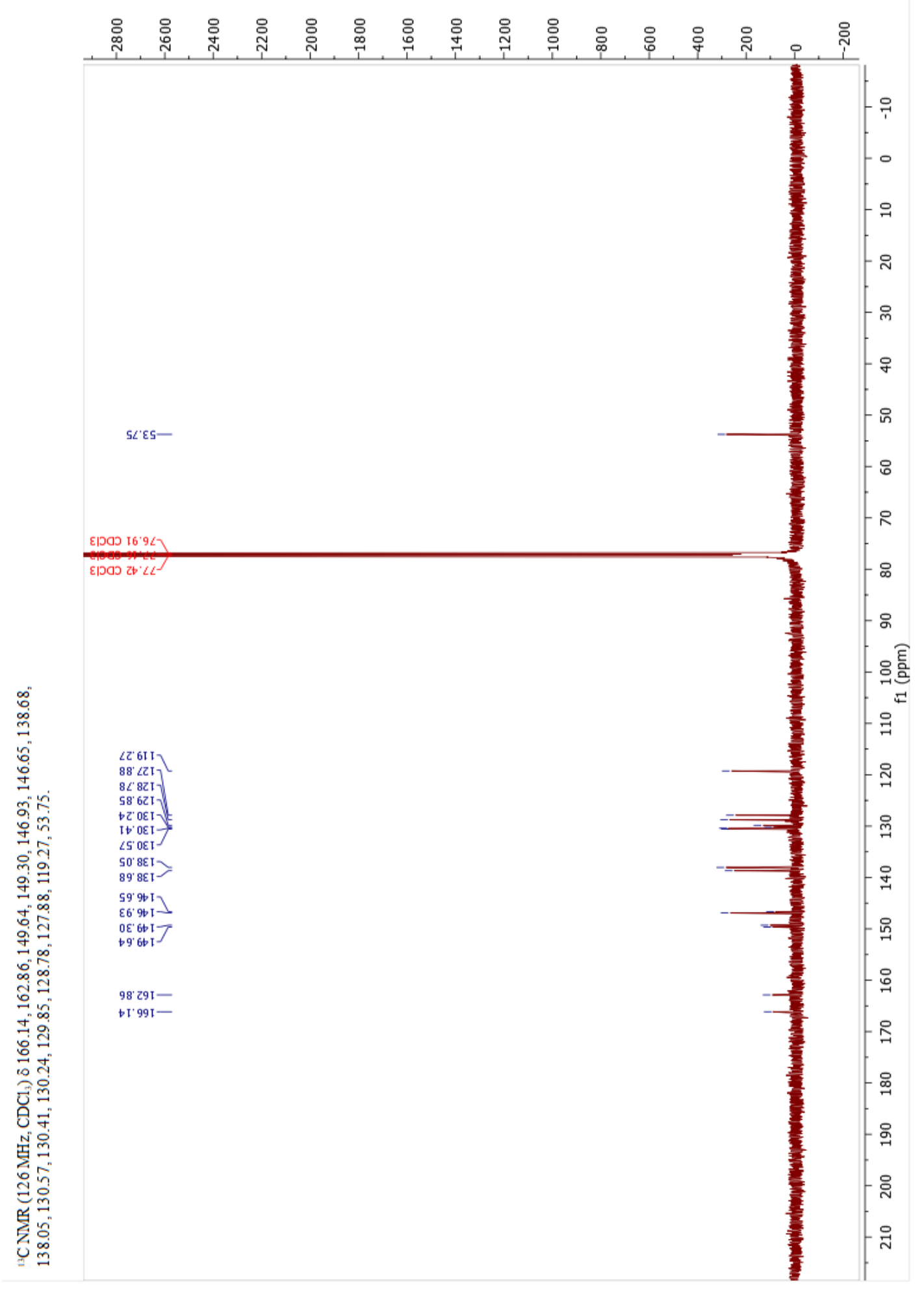


<smiles>O=C(Nc1nccnc1C(=O)O)c1ccc2ccccc2n1</smiles>

3-(Quinoline-2-carboxamido)pyrazine-2-carboxylic acid (19)

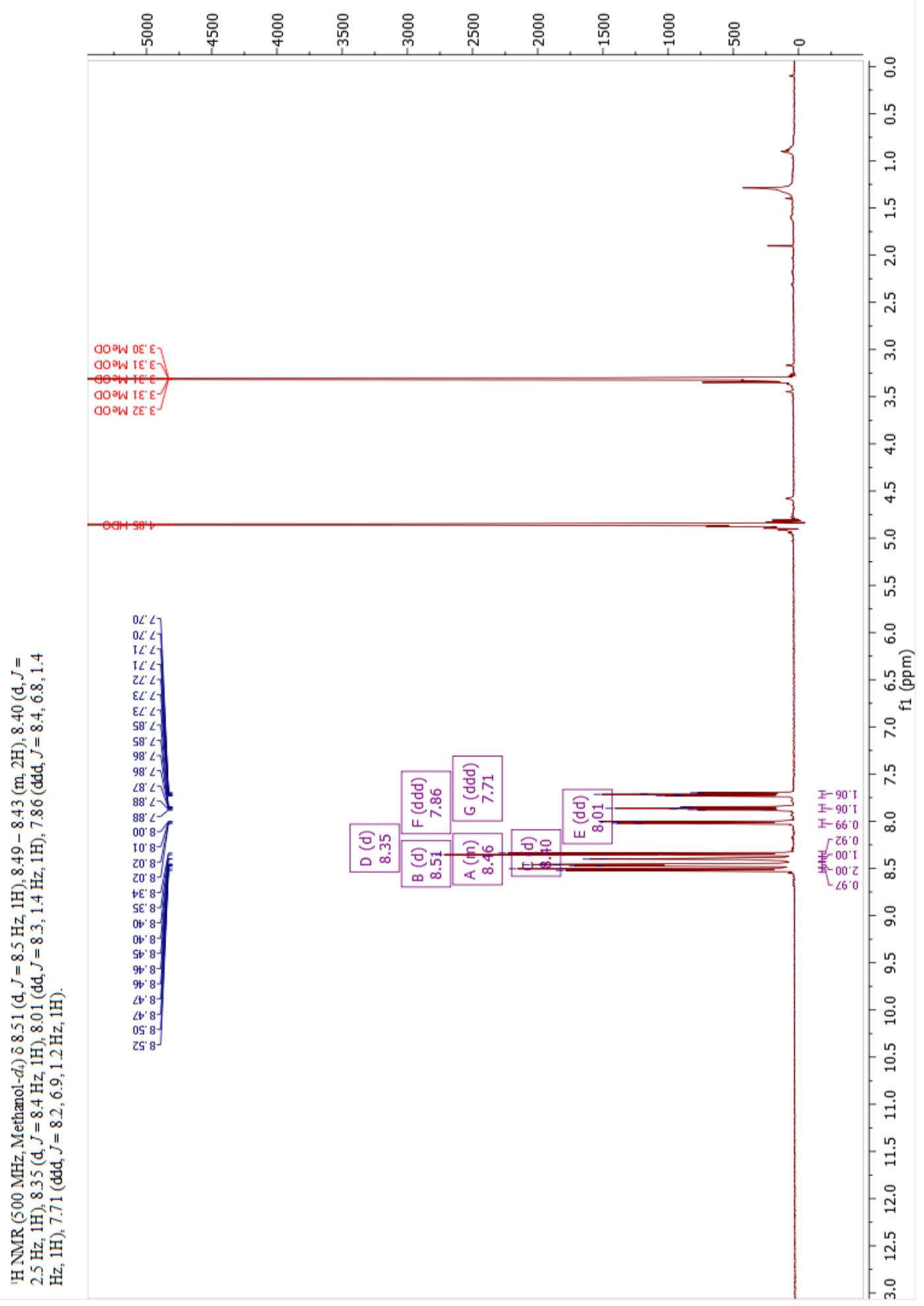




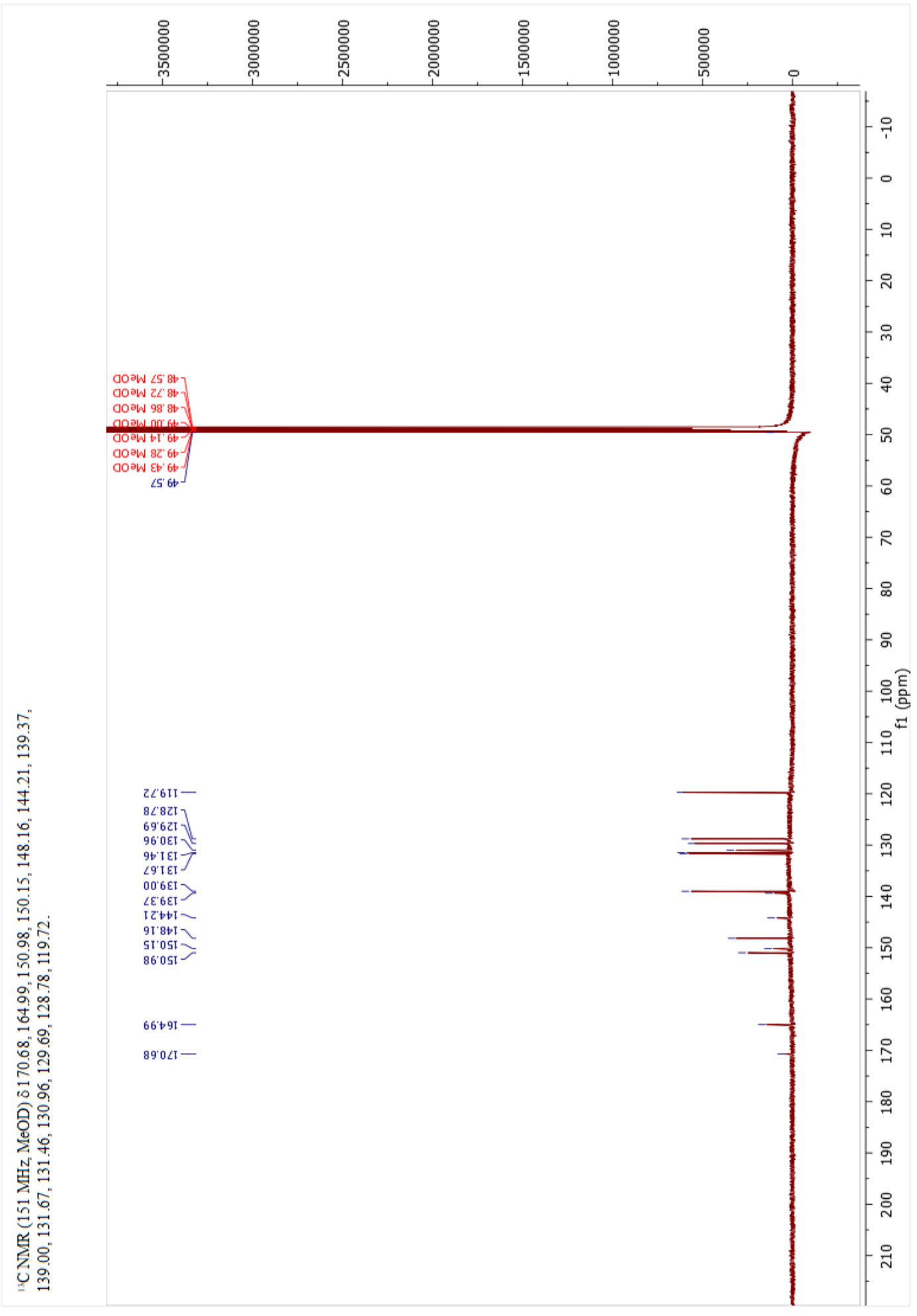




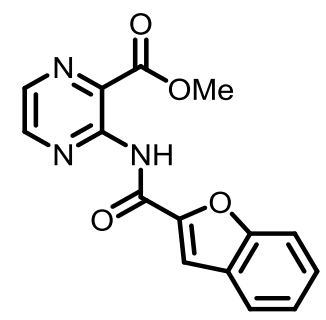

Methyl 3-(benzofuran-2-carboxamido)pyrazine-2-carboxylate (20-OMe)

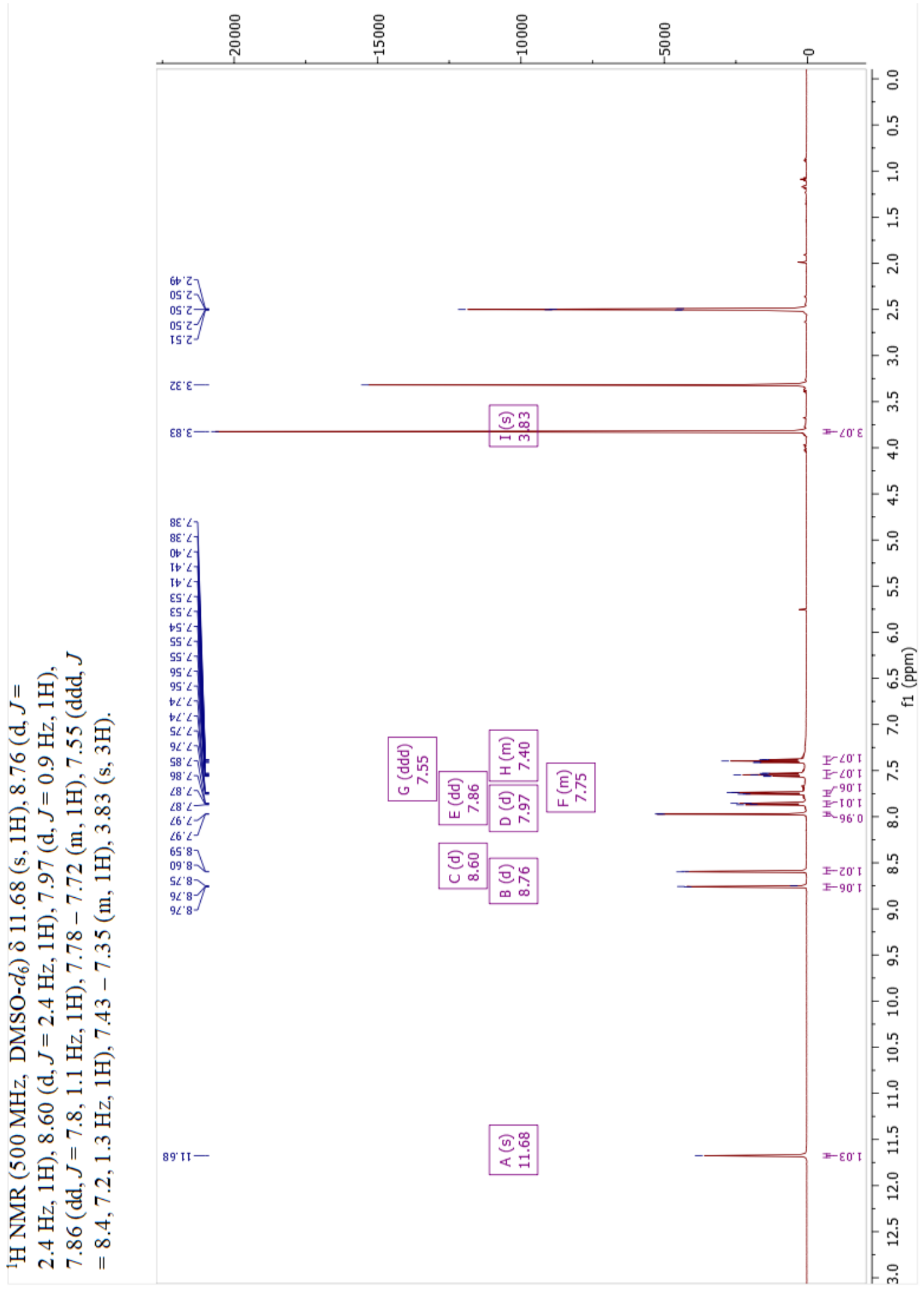




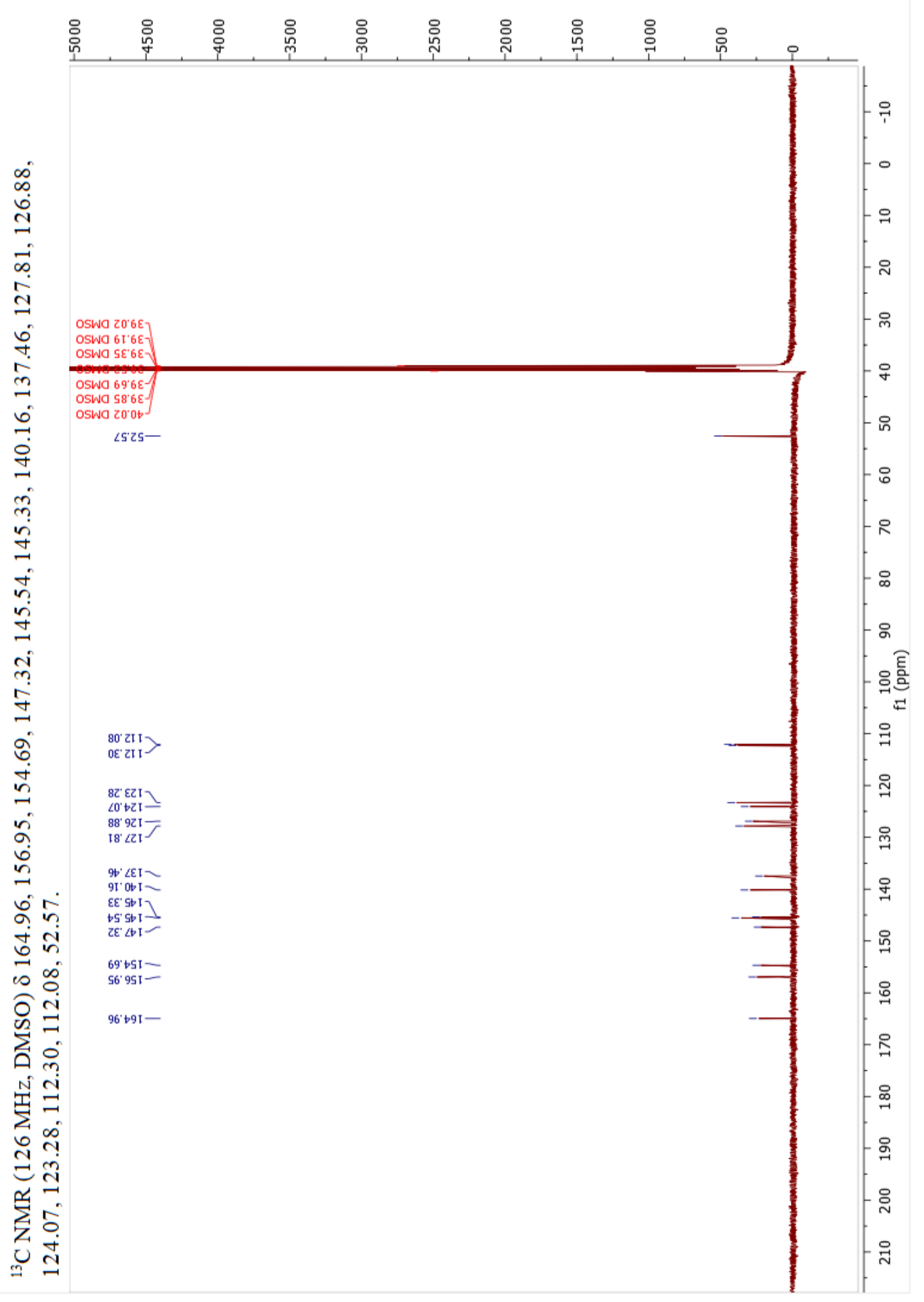




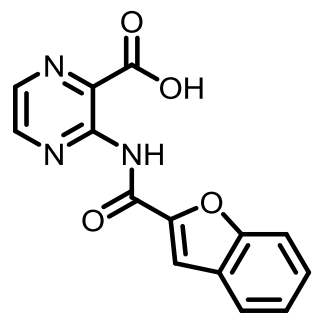

3-(Benzofuran-2-carboxamido)pyrazine-2-carboxylic acid (20)

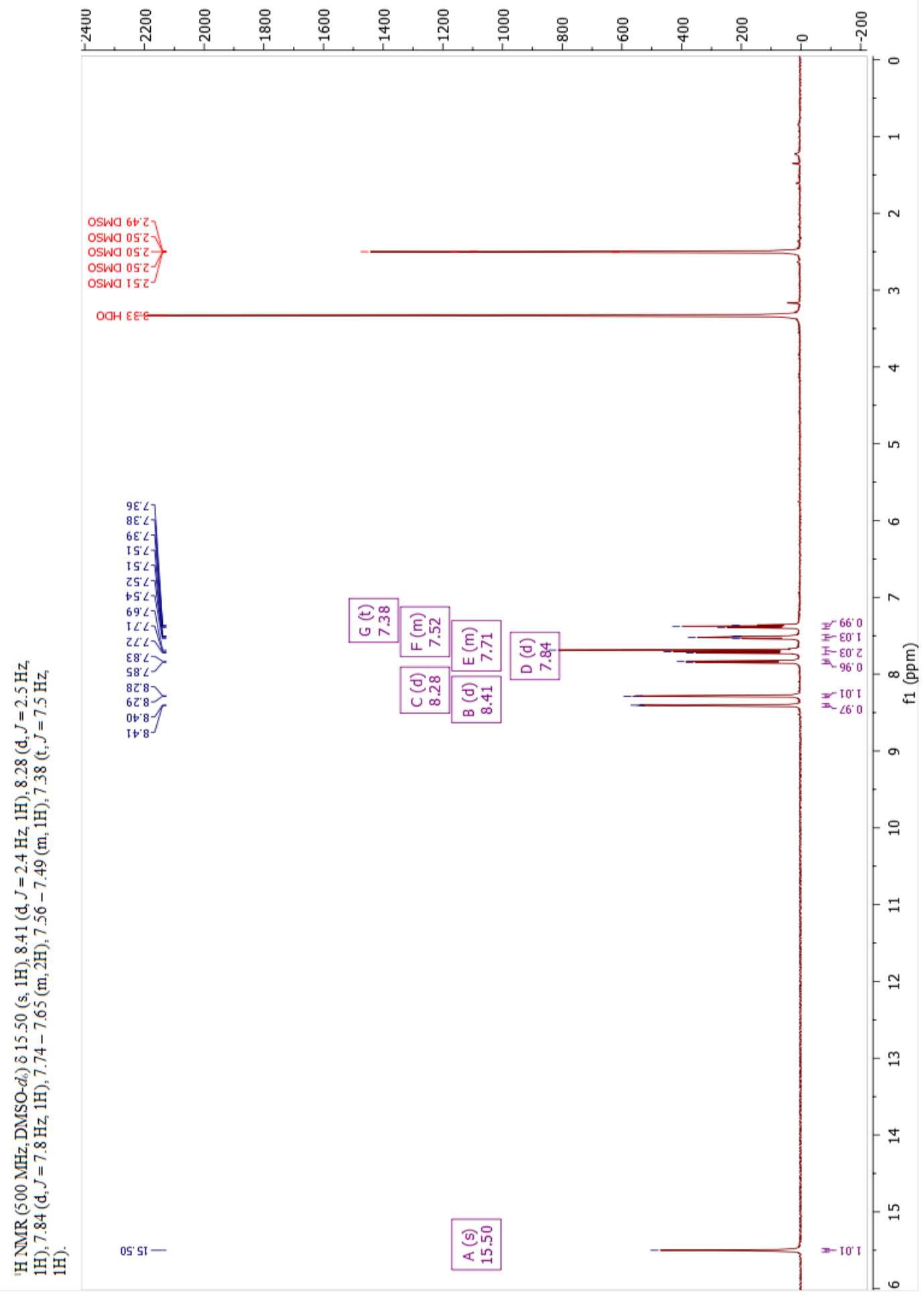




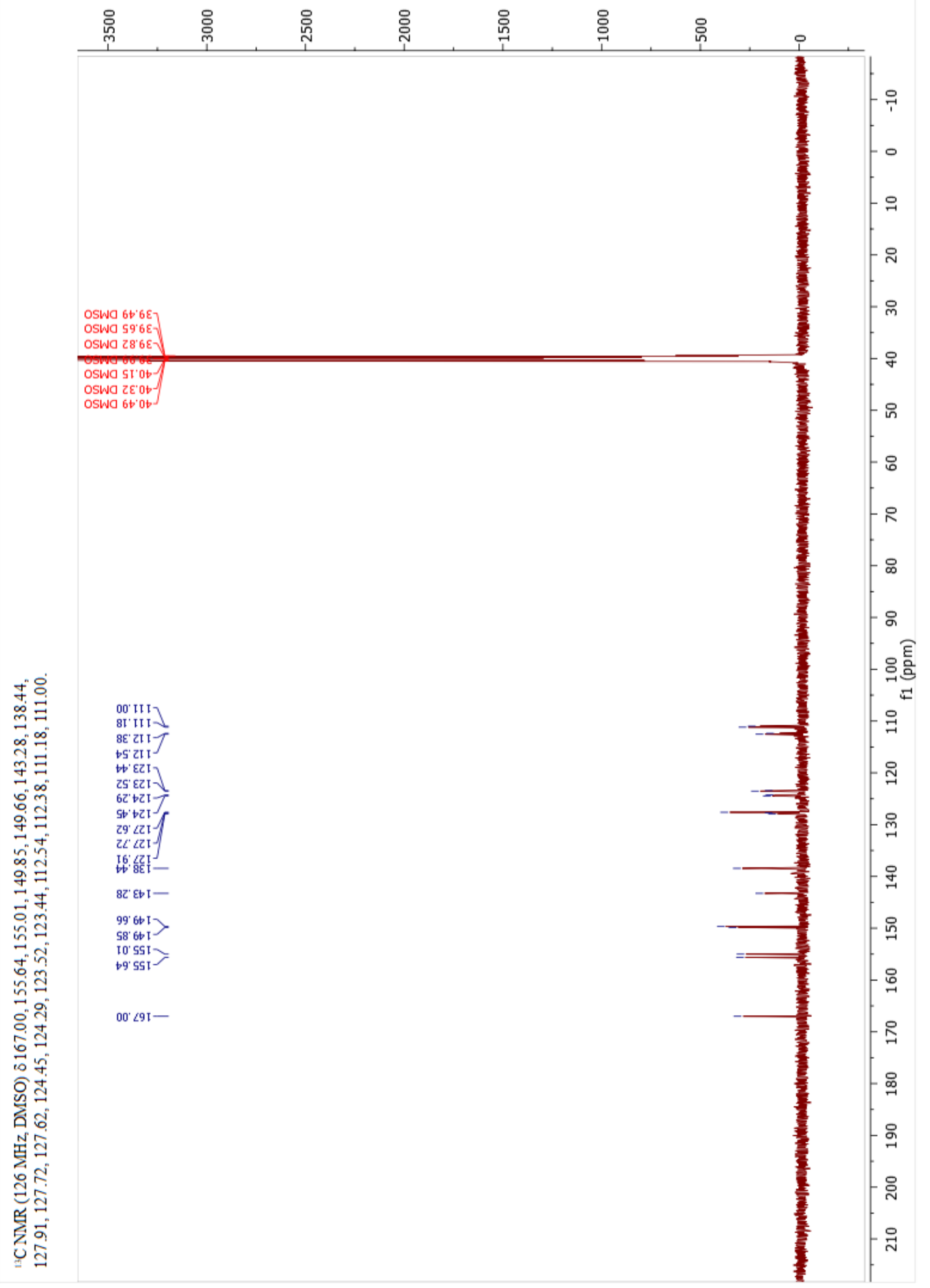

